Federal Reserve Bank of Minneapolis

Research Department Staff Report 262

June 1999

\title{
Risk Sharing: Private Insurance Markets or Redistributive Taxes?
}

\author{
Dirk Krueger* \\ Federal Reserve Bank of Minneapolis \\ and University of Minnesota
}

Fabrizio Perri*

New York University

\begin{abstract}
We explore the welfare consequences of different taxation schemes in an economy where agents are debt-constrained. If agents default on their debt, they are banned from future credit markets, but retain their private endowments which are subject to income taxation. A change in the tax system changes the severity of punishment from default and, hence, leads to a limitation of possible risk sharing via private contracts. The welfare consequences of a change in the tax system depend on the relative magnitudes of increased risk sharing forced by the new tax system and the reduced risk sharing in private insurance markets. We quantitatively address this issue by calibrating an artificial economy to US income and tax data. We show that for a plausible selection of the structural parameters of our model, the change to a more redistributive tax system leads to less risk sharing among individuals and lower ex-ante welfare.

*We would like to thank Andrew Atkeson, V.V. Chari, Hal Cole, Patrick Kehoe, Tim Kehoe, Narayana Kocherlakota, Edward Prescott and seminar participants at the Minneapolis and Richmond FED, SED Meetings in Philadelphia, BU, Chicago, ECARE, Northwestern, NYU, Pompeu Fabra, Rochester, Tel Aviv, UCL, UCLA, UPenn, Wharton and Wisconsin for helpful comments and suggestions. Both authors acknowledge financial support from the Alfred P. Sloan Foundation through a doctoral dissertation fellowship. All remaining errors are our own. The views expressed herein are those of the authors and not necessarily those of the Federal Reserve Bank of Minneapolis or the Federal Reserve System.
\end{abstract}




\section{Introduction}

A long standing discussion in macroeconomics and public finance evolves around the desirability of redistributive income taxes as a risk sharing device against idiosyncratic income uncertainty. ${ }^{1}$ The insights that economic theory provides depend on the assumptions about the structure of private insurance markets. On one hand, if these markets are complete, in the sense that agents can insure against any contingency and thus achieve perfect risk sharing, then redistributive income taxes provide no additional insurance. On the other hand, if private insurance markets are nonexistent or incomplete, redistributive taxes might provide additional insurance not available otherwise and therefore increase ex-ante welfare.

Recent empirical studies (see Hayashi et al. (1996), Attanasio and Davis (1996)) have seriously challenged the complete markets assumption mostly on the ground that risk sharing among individuals is not perfect ${ }^{2}$. As Hayashi et al. conclude: "Our result there is no full insurance even among related households should serve as a final blow to the complete markets paradigm". Attempting to reconcile the theory with the empirical observation of incomplete risk sharing, there is a large body of literature that introduces some form of market incompleteness into the economic environment. One fraction of this literature (see Bewley (1986), Kimball and Mankiw (1989), Huggett (1993), Aiyagari (1994), among others) exogenously assumes that some insurance markets are nonexistent (usually it is assumed that agents can only self-insure through a single uncontingent bond and they are borrowing constrained). Another fraction derives market incompleteness from informational frictions underlying the phenomena of adverse selection and moral hazard (see Cole and Kocherlakota (1998) and their review of this extensive literature).

Both approaches do not explicitly capture what we believe is a crucial aspect of redistributive taxation as a risk sharing device: the fact that a particular tax regime is in place might affect the incentives private agents have to enter into private insurance contracts and thus the form and extent of market incompleteness. It is obvious that the first approach

\footnotetext{
${ }^{1}$ See Varian (1980) and his review of the related literature. The insurance effect of redistributive income taxes may also invalidate the Ricardian Equivalence theorem, see Chan (1983), Barsky et al. (1986) and Kimball and Mankiw (1989).

${ }^{2}$ As first highlighted by Mehra and Prescott (1985), another empirical dimension along which the complete markets model does not perform well is the explanation of the main stylized facts regarding asset prices.
} 
is silent about this connection since market incompleteness is not derived from underlying primitives but rather exogenously assumed. In the second approach fiscal policy can affect the degree of market incompleteness, but only under the assumption of informational asymmetry between the government and private agents. However, every policy that leads to the same revelation of information to private agents has the same effect on the degree of market incompleteness. In this sense this approach does not provide a natural link between market incompleteness and redistributive income taxation in particular. Also, recent empirical work by Attanasio and Davis (1996) seems to indicate that "evidence against the consumption insurance hypothesis involves publicly observed shocks, hence cannot be rationalized as a consequence of unobserved shocks in environments with informationally constrained insurance" (p. 1259).

We therefore explore an alternative way of deriving endogenous market incompleteness that relies on limited enforceability of private contracts. We follow the approach of Kehoe and Levine $(1993,1998)$ and assume that private contracts can be enforced only by the threat of exclusion from future credit markets. Tax liabilities, however, are not subject to this enforcement problem as we assume that the penalty for defaulting on tax payments can be made prohibitively large by the government. If agents default on their private debt, they are banned from future intertemporal trade, but retain their private (labor) endowments which are still subject to income taxation. We impose individual rationality constraints on agents guaranteeing no default in equilibrium and we solve for the efficient consumption distribution across agents. A change in the tax system changes the severity of punishment from default by altering the utility an agent can attain without access to credit markets. We demonstrate that a change to a more redistributive tax system leads to a restriction of the set of contracts that are individually rational. In an economy that is characterized by uncertainty with respect to individual endowments, this restriction leads to a limitation of possible risk sharing via private contracts. The welfare consequences of a change in the tax system depend on the relative magnitudes of increased risk sharing forced by the new tax system and the reduced risk sharing in private insurance markets. We quantitatively address this issue by designing an artificial economy calibrated to US income and tax data. We find that for a reasonable selection of the structural parameters of our model, the change to a more redistributive tax 
system leads to less risk sharing among individuals and, hence, lower ex-ante welfare.

Other authors have studied economies with debt constraints to analyze a variety of issues (See Kocherlakota (1996) and Alvarez and Jermann (1998a,b) among others). These authors consider economies with two (types of) agents in which heterogeneity is somehow limited. The main methodological contribution of this work is the analysis of a debt constrained economy with a continuum of agents facing idiosyncratic uncertainty. Therefore a steady state of our economy is characterized by a non-degenerate consumption distribution. We believe that this feature of the model is necessary to analyze issues of income distribution and risk sharing in a quantitatively meaningful way, especially as the quantitative implications for the economy with a continuum of agents turn out to be quite different from the economy with only two agents. But it is also this feature of the model that leads to considerable theoretical and computational complications in solving the model. To this end we draw heavily on the work of Atkeson and Lucas $(1992,1995)$ who study efficient allocations in an economy with a continuum of agents and private information. We will describe their dual approach to characterize efficient allocations in more detail in the next section.

The paper is organized as follows. In section 2 we lay out the model environment. In section 3 we define an efficient allocation with debt constraints and we show how to make the problem of solving for efficient allocations recursive. We then prove the existence of a stationary solution to the recursive problem. Section 4 contains a discussion of the parameterization we chose for our quantitative exercises. In section 5 we present our quantitative results and in section 6 we compare our results with those from a model with exogenous incomplete markets. Section 7 concludes and figures, proofs and details about the computational procedure are contained in the appendix.

\section{The Environment}

\section{A. Consumers}

There is a continuum of consumers of measure 1. The consumers have preferences over consumption streams given by

(1) $U\left(\left\{c_{t}\right\}_{t=0}^{\infty}\right)=(1-\beta) E_{0}\left[\sum_{t=0}^{\infty} \beta^{t} u\left(c_{t}\right)\right]$ 
The period utility function $u: \Re_{+} \rightarrow D \subseteq \Re$ is assumed to be strictly increasing, strictly concave, twice differentiable and satisfies the Inada conditions. Its inverse is denoted by $C: D \rightarrow \Re_{+}$. Hence $C(u)$ is the amount of the consumption good necessary to yield period utility $u$. Let $\bar{D}=\sup (D)$, where it is understood that $\bar{D}$ could be infinity. An individual has a stochastic endowment process $e \in E$, a finite set with cardinality $N$, that follows a Markov process with transition probabilities $\pi\left(e^{\prime} \mid e\right)$. For each consumer the transition probabilities are assumed to be the same. We assume a law of large numbers, so that the fraction of agents facing shock $e^{\prime}$ tomorrow with shock $e$ today in the population is equal to $\pi\left(e^{\prime} \mid e\right)$. We assume that $\pi\left(e^{\prime} \mid e\right)$ has a unique invariant measure $\Pi($.$) . We denote by e_{t}$ the current period endowment and by $e^{t}=\left(e_{0}, . ., e_{t}\right)$ the history of realizations of endowment shocks; also $\pi\left(e^{t} \mid e_{0}\right)=\pi\left(e_{t} \mid e_{t-1}\right) \cdots \pi\left(e_{1} \mid e_{0}\right)$. We use the notation $e^{s} \mid e^{t}$ to mean that $e^{s}$ is a possible continuation of endowment shock realization $e^{t}$. We also assume that at date 0 (and hence at every date), the measure over current endowment is given by $\Pi($.$) , so aggregate endowment is$ constant over time. At date 0 agents are distinguished by their initial asset holdings, $a_{0}$, and by the their initial shock $e_{0}$. Let $\Phi_{0}$ be the joint measure of initial assets and shocks. Most of the theoretical results presented here depend on the assumption that endowment shocks are uncorrelated across time for each agent. In our numerical exercises we will relax this assumption, although some theoretical results cannot be proved for the more general case in which individual endowment processes are Markov over time.

\section{B. Government}

The government uses taxes to finance a constant amount of public spending $g$ in every period that yields no utility to consumers. The government specifies a tax policy $\tau\left(e_{t}\right)$ that is constant over time. We take the government policies $g, \tau($.$) as exogenously given. For an$ individual we let $y_{t}=e_{t}\left(1-\tau\left(e_{t}\right)\right)$ be the after-tax income. Since the function $\tau($.$) does$ not depend on time, for a given $\tau($.$) there is a one to one mapping between pre-tax and$ after-tax endowments. So from now on we let $y \in Y$, a finite set with cardinality $N$, denote an individual's generic after-tax endowment, following the Markov process $\pi$ with invariant distribution $\Pi$ and denote by $y^{t}=\left(y_{0}, \ldots y_{t}\right)$ a history of after-tax endowment shocks. We 
restrict the government policies $g, \tau($.$) to satisfy period-by-period budget balance$

(2) $g=\int e_{t} \tau\left(e_{t}\right) d \Pi$.

With this assumption resource feasibility (as defined below) for this economy simply states that the sum of all agents' consumption has to be less or equal than the sum over all individuals' after-tax endowment. Therefore, once $g, \tau($.$) are fixed and hence the after-tax endowment$ process is specified, we can carry out the subsequent analysis without explicit consideration of the government.

\section{Continuing Participation Constraints}

Consumers can trade a full set of state-contingent commodities. ${ }^{3}$ A consumption allocation $\left\{c\left(a_{0}, y^{t}\right)\right\}$ specifies how much an agent of type $\left(a_{0}, y_{0}\right)$ consumes who experienced a history of endowment shocks (net of taxes) $y^{t}$. Individuals, at any point in time, have the option to renege on existing contracts. The only punishment for doing so, and hence the only enforcement mechanism for contracts, is that agents that choose to default on their contracts are banned from future credit markets but are allowed to self-insure by saving at an interest rate $r$. In particular the expected utility for an agent who defaults after history $y^{t}$ is given by:

$$
\begin{aligned}
U_{t}^{A u t}\left(y^{t} ; r\right)= & \max _{\left\{c_{\tau}\left(y^{\tau}\right), a_{\tau+1}\left(y^{\tau}\right)\right\}}(1-\beta) \sum_{\tau \geq t} \sum_{y^{\tau} \mid y^{t}} \beta^{\tau-t} \pi\left(y^{\tau} \mid y^{t}\right) u\left(c_{\tau}\left(y^{\tau}\right)\right) \\
& \text { s.t. } \\
c_{\tau}\left(y^{\tau}\right)+a_{\tau+1}\left(y^{\tau}\right)= & y_{\tau}+(1+r) a_{\tau}\left(y^{\tau-1}\right) \quad \forall \tau \geq t, y^{\tau} \mid y^{t} \\
a_{\tau+1}\left(y^{\tau}\right) \geq & 0 \quad \text { with } a_{t}\left(y^{t-1}\right)=0 \text { given }
\end{aligned}
$$

Given the Markov structure of the endowment process it is clear that $U_{t}^{A u t}\left(y^{t} ; r\right)=U^{A u t}\left(y_{t} ; r\right)$. Standard dynamic programming techniques can be used to show that for $1+r<\frac{1}{\beta}, U^{A u t}\left(y_{t} ; r\right)=$ $U\left(0, y_{t}\right)$, where $U$ is the solution to the functional equation (for details see Huggett (1993),

\footnotetext{
${ }^{3}$ Trade occurs with financial intermediaries. See the decentralization section for details.
} 
theorem 1)

$$
\begin{aligned}
U(a, y)= & \max _{c, a^{\prime} \geq 0}(1-\beta) u(c)+\beta \sum_{y^{\prime}} \pi\left(y^{\prime} \mid y\right) U\left(a^{\prime}, y^{\prime}\right) \\
& \text { s.t. } \\
c+a^{\prime}= & y+(1+r) a
\end{aligned}
$$

It is obvious that $U_{t}^{A u t}\left(y_{t} ; r\right)$ is strictly increasing in $y_{t}$ (as long as the income shocks are uncorrelated or positively correlated over time). An important fact is that $U_{t}^{A u t}\left(y_{t} ; r\right)$ is continuous and strictly increasing in $r$. This is due to the fact that the constraint set in the functional equation is continuous and strictly increasing in $r$, since $a \geq 0$.

Individuals have no incentive to default, at any point in time and any contingency, if and only if an allocation satisfies following continuing participation or debt constraints

$$
(1-\beta)\left(u\left(c\left(a_{0}, y^{t}\right)\right)+\sum_{s>t} \sum_{y^{s} \mid y^{t}} \beta^{s-t} \pi\left(y^{s} \mid y^{t}\right) u\left(c\left(a_{0}, y^{s}\right)\right)\right) \geq U_{t}^{A u t}\left(y_{t} ; r\right) \quad \forall y^{t}
$$

Since there is no private information and markets are complete, default will not happen in equilibrium as nobody would offer a contract with an individual for a contingency at which this individual would later default with certainty.

Notice that our specification of the debt constraint is more general than the one introduced by Kehoe and Levine(1993) in which agents who default are not allowed to save. If $r=-1$ our model is equivalent to theirs and the right hand side of the debt constraint reduces to

$$
U_{t}^{A u t}\left(y_{t} ;-1\right)=(1-\beta)\left(u\left(y_{t}\right)+\sum_{s>t} \sum_{y^{s} \mid y^{t}} \beta^{s-t} \pi\left(y^{s} \mid y^{t}\right) u\left(y_{s}\right)\right)
$$

In the next sections, whenever there is no danger of ambiguity, we omit the dependence of $U_{t}^{\text {Aut }}$ on $r$. We now first discuss how to compute efficient allocations, then how to decentralize them. 


\section{Characterization of Efficient Allocations}

In order to characterize efficient allocations (for given policies $g, \tau($.$) ) we proceed in$ four steps: we first define what we mean by an efficient allocation. We go on to show that one can find efficient allocations by solving a number of appropriate social planner problems. We then show that solving the social planner problems is equivalent to solving a specific functional equation and finally we discuss how to solve the functional equation. This discussion uses ideas and results developed by Atkeson and Lucas $(1992,1995)$ extensively. Proofs, however, might differ substantially since these authors analyze a private information economy, and are therefore included in the appendix even when the proof strategy resembles theirs closely.

\section{A. Definition of Efficient Allocations}

The key insight of Atkeson and Lucas is to analyze the problem of finding efficient allocation in terms of state contingent utility promises rather than state contingent consumption. Since the continuing participation constraints are in utility space this approach is particularly useful for our problem. Instead of being indexed by initial assets and endowment shock, now individuals are indexed by initial entitlements to expected discounted utility at period $0, w_{0}$ and initial endowment shocks, $y_{0}$. Let $\Phi_{0}$ be the period 0 joint measure over $\left(w_{0}, y_{0}\right)$. An allocation is then a sequence $\left\{h_{t}\left(w_{0}, y^{t}\right)\right\}_{t=0}^{\infty}$ that maps initial entitlements $w_{0}$ and sequences of shocks $y^{t}$ into levels of current utility ${ }^{4}$ in period $t$. Here $h_{t}\left(w_{0}, y^{t}\right)$ is the current period utility that an agent of type $\left(w_{0}, y_{0}\right)$ receives if she experienced a history of endowment shocks $y^{t}$. Note that $c\left(a_{0}, y^{t}\right)=C\left(h_{t}\left(w_{0}, y^{t}\right)\right)$ for an agent whose utility entitlement $w_{0}$ corresponds to initial asset holdings $a_{0}$, where $C$ is the inverse of the period utility function as defined in Section 2. We will now define the concepts of constrained feasibility and efficiency in this environment.

For any allocation $h=\left\{h_{t}\left(w_{0}, y^{t}\right)\right\}_{t=0}^{\infty}$ define

$$
U_{t}\left(w_{0}, y^{t}, h\right)=(1-\beta)\left(h_{t}\left(w_{0}, y^{t}\right)+\sum_{s>t}^{\infty} \sum_{y^{s}} \beta^{s-t} \pi\left(y^{s}\right) h_{t}\left(w_{0}, y^{s}\right)\right)
$$

\footnotetext{
${ }^{4}$ The relation between $h_{t}$ and the period utility derived from consuming $c_{t}$ is given by $h_{t}\left(w_{0}, y^{t}\right)=$ $u\left(c\left(a_{0}, y^{t}\right)\right)$.
} 
Equation 4 defines the continuation utility from an allocation $h$ of agent of type $\left(w_{0}, y_{0}\right)$ from date $t$ and shock history $y^{t}$ onwards.

DeFinition 1. An allocation $\left\{h_{t}\left(w_{0}, y^{t}\right)\right\}_{t=0}^{\infty}$ is constrained feasible with respect to a joint distribution over utility entitlements and initial endowments, $\Phi_{0}$, if for each $\left(w_{0}, y_{0}\right) \in \operatorname{supp}\left(\Phi_{0}\right)$

$$
\begin{gathered}
w_{0}=U_{0}\left(w_{0}, y_{0}, h\right) \\
U_{t}\left(w_{0}, y^{t}, h\right) \geq U_{t}^{A u t}\left(y_{t}\right) \quad \forall y^{t} \\
\lim _{t \rightarrow \infty} \beta^{t} \sup _{y^{t}} U_{t}\left(w_{0}, y^{t}, h\right)=0 \\
\sum_{y^{t}} \int\left(C\left(h_{t}\left(w_{0}, y^{t}\right)\right)-y_{t}\right) \pi\left(y^{t} \mid y_{0}\right) d \Phi_{0} \leq 0 . \quad \forall t
\end{gathered}
$$

We call equation (5) the promise keeping constraint: the allocation delivers utility $w_{0}$ to agents entitled to $w_{0}$. Equations (6) are the continuing participation constraints, just posed in utility space. ${ }^{5}$ Equation (7) is a boundedness condition that assures that continuation utility goes to zero in the time limit. Finally equation (8) is the resource feasibility condition, requiring aggregate consumption in every period to be less or equal than aggregate endowment in that period. Now we can define the concept of efficiency in this environment, due to Atkeson and Lucas (1995).

Definition 2. An allocation $\left\{h_{t}\left(w_{0}, y^{t}\right)\right\}_{t=0}^{\infty}$ is efficient with respect to $\Phi_{0}$ if

- It is constrained feasible with respect to $\Phi_{0}$.

- There does not exist another allocation $\left\{\hat{h}_{t}\left(w_{0}, y^{t}\right)\right\}_{t=0}^{\infty}$ that is constrained-feasible with

\footnotetext{
${ }^{5}$ Note that a $\Phi_{0}$ that puts positive mass on $\left(w_{0}, y_{0}\right)$ that satisfies

$$
w_{0}<u\left(y_{0}\right)+\sum_{s>0} \sum_{y^{s} \mid y_{0}} \beta^{s} \pi\left(y^{s} \mid y_{0}\right) u\left(y_{s}\right)
$$

does not permit a constraint feasible allocation as promise keeping and debt constraints are mutually exclusive in this case. I restrict attention to $\Phi_{0}$ with the property that only initial utility entitlements at least as big as the utilty from autarky have positive mass.
} 
respect to $\Phi_{0}$ and such that

(9) $\sum_{y^{t}} \int C\left(\hat{h}_{t}\left(w_{0}, y^{t}\right)\right) \pi\left(y^{t} \mid y_{0}\right) d \Phi_{0}<\sum_{y^{t}} \int C\left(h_{t}\left(w_{0}, y^{t}\right)\right) \pi\left(y^{t} \mid y_{0}\right) d \Phi_{0}$ for some $t$

The definition basically says that a utility allocation is efficient if it attains the utility promises made by $\Phi_{0}$ in an individually rational and resource feasible way and there is no other allocation that does so with less resources.

\section{B. A Component Social Planner's Problem $(C P P)$}

Is there an operational way to solve for efficient allocations? Consider now the problem of a social planner faced with a sequence of intertemporal shadow prices $\left\{R_{t}\right\}_{t=0}^{\infty}$ to minimize the value of resources needed to deliver expected discounted utility of $w_{0}$ to an individual with initial endowment given by $y_{0}$. The planner chooses $\left\{h_{t}\left(w_{0}, y^{t}\right)\right\}_{t=0}^{\infty}$ to solve the problem

$$
\begin{aligned}
& W\left(w_{0}, y_{0}\right) \\
= & \min \left(1-\frac{1}{R_{0}}\right) C\left(h_{0}\left(w_{0}, y_{0}\right)\right)+\sum_{t=1}^{\infty}\left(1-\frac{1}{R_{t}}\right) \prod_{s=0}^{t-1}\left(\frac{1}{R_{s}}\right) \sum_{y^{t} \mid y_{0}} C\left(h_{t}\left(w_{0}, y^{t}\right)\right) \pi\left(y^{t} \mid y_{0}\right)
\end{aligned}
$$

subject to (5), (6), and (7). One obtains the following

TheOREM 1. (Atkeson and Lucas (1995)) If there exist allocations $\left\{h_{t}\left(w_{0}, y^{t}\right)\right\}$, shadow prices $\left\{R_{t}\right\}$ and distribution $\Phi_{0}$ such that:

1. Given $\left\{R_{t}\right\}_{t=0}^{\infty}$, for each $\left(w_{0}, y_{0}\right) \in \operatorname{supp}\left(\Phi_{0}\right),\left\{h_{t}\left(w_{0}, y^{t}\right)\right\}$ solves $C P P$

2. Feasibility (Equation 6 ) holds with equality for every $t$

3 .

$$
1-\frac{1}{R_{0}}+\sum_{t=1}^{\infty}\left(1-\frac{1}{R_{t}}\right) \prod_{s=0}^{t-1}\left(\frac{1}{R_{s}}\right)<\infty
$$

then the allocation is efficient with respect to $\Phi_{0}$.

\section{Proof. See Appendix}

This theorem gives an operational method for solving for efficient allocations. Given a set of shadow prices satisfying 11 one has to solve a minimization problem for allocations 
$\left\{h_{t}\left(w_{0}, y^{t}\right)\right\}$. Then one has to check whether the resource constraints are satisfied, and if not, pick different shadow prices. In order to make $C P P$ recursive, however, we have to restrict ourselves to stationary allocations. Define $\Phi_{t}$ to be the joint measure over endowment shocks $y_{t}$ and continuation utilities $U_{t}\left(w_{0}, y^{t}, h\right)$ for a given allocation. An allocation is stationary if $\Phi_{t}=\Phi_{0}=\Phi$. Stationarity also suggests the sequence of shadow prices $\left\{R_{t}\right\}$ to be constant at some $R$. We want to find such $R$, a corresponding allocation $\left\{h_{t}^{R}\left(w_{0}, y^{t}\right)\right\}$ and an initial distribution over utility entitlements and endowment shocks, $\Phi_{R}$ that satisfies the hypothesis of Theorem 1. and is stationary. Once we restrict ourselves to these types of allocations we can solve for them recursively. This assertion is demonstrated in the next subsections.

\section{Recursive Formulation of the Problem}

For constant $R \in\left(1, \frac{1}{\beta}\right)$, consider the following functional equation $(F E)$ problem. We will show in Theorem 3. that the optimal policies of the functional equation induce an allocation that solves $C P P$. Individual state variables are the promise to expected discounted utility that an agent enters the period with, $w$, and the current income shock $y$. The planner chooses how much current period utility to give to the individual, $h$, and how much to promise her for the future, $g_{y^{\prime}}$, conditional on her next periods endowment realization $y^{\prime}$. The functional equation associated with the planner's problem is:

$$
\begin{aligned}
& V(w, y)= \inf _{\substack{h,\left\{g_{y^{\prime}}\right\}_{y^{\prime} \in Y} \\
\text { s.t }}}\left\{\left(1-\frac{1}{R}\right) C(h(w, y))+\frac{1}{R} \sum_{y^{\prime} \in Y} \pi\left(y^{\prime} \mid y\right) V\left(g_{y^{\prime}}(w, y), y^{\prime}\right)\right\} \\
& w=(1-\beta) h(w, y)+\beta \sum_{y^{\prime} \in Y} \pi\left(y^{\prime} \mid y\right) g_{y^{\prime}}(w, y) \\
& g_{y^{\prime}}(w, y) \geq U^{\text {Aut }}\left(y^{\prime}\right) \quad \forall y^{\prime} \in Y
\end{aligned}
$$

where $V(w, y)$ is the resource cost for the planner to provide an individual with expected utility $w$ when the individual's endowment is $y$ and the intertemporal shadow price of resources 
for the planner is $\frac{1}{R}$. The cost consists of the cost for utility delivered today, $\left(1-\frac{1}{R}\right) C(h)$, and expected cost from tomorrow on, $\sum_{y^{\prime}} \pi\left(y^{\prime} \mid y\right) V\left(g_{y^{\prime}}, y^{\prime}\right)$, discounted to today. ${ }^{6}$

Equation (13) is the promise-keeping constraint: an individual that is entitled to $w$ in fact receives utility $w$ through the allocation rules $\left\{h(., .), g_{y^{\prime}}(., .)\right\}_{y^{\prime} \in Y}$. The continuing participation constraints in equation (14) state that the social planner for each state tomorrow has to guarantee individuals an expected utility promise at least as high as obtained with the autarkic allocation. The utility in autarky is given as the solution to the following functional equation:

$$
U^{A u t}(y)=(1-\beta) u(y)+\beta \sum_{y^{\prime} \in Y} \pi\left(y^{\prime} \mid y\right) U^{A u t}\left(y^{\prime}\right)
$$

We now make the following assumptions on the individual endowment process for the rest of this section:

1. $\pi\left(y^{\prime} \mid y\right)=\pi\left(y^{\prime}\right)$ for every $y^{\prime}, y \in Y$, i.e. endowment shocks are independently distributed across time.

2. $\pi(y)>0$, for all $y \in Y$

ReMARK 1 . The fact that $\pi$ is a stochastic matrix implies that the functional equation (15) has a unique solution $\left\{U^{A u t}(y)\right\}_{y \in Y}$. Assumption 1 immediately implies that if $\bar{y}>y$, then $U^{A u t}(\bar{y})>U^{A u t}(y)$.

\footnotetext{
${ }^{6}$ The prices for resources in sequential component planner problem were

$$
p_{t}=\left(1-\frac{1}{R_{t}}\right) \prod_{s=0}^{t-1} \frac{1}{R_{s}} .
$$
}

Note that this implies that

$$
\sum_{t} p_{t}=1
$$

With $R_{t}=R$ for all $t$ we have

$$
\begin{aligned}
p_{0} & =\left(1-\frac{1}{R}\right) \\
\frac{p_{t+1}}{p_{t}} & =\frac{1}{R} .
\end{aligned}
$$


REMARK 2. Assumption 1 implies that one can write the functional equation as

$$
\begin{array}{r}
V(w)=\inf _{h, g_{y^{\prime}}}\left\{\left(1-\frac{1}{R}\right) C(h(w))+\frac{1}{R} \sum_{y^{\prime} \in Y} \pi\left(y^{\prime}\right) V\left(g_{y^{\prime}}(w)\right)\right\} \\
\text { s.t } \\
w=(1-\beta) h(w)+\beta \sum_{y^{\prime} \in Y} \pi\left(y^{\prime}\right) g_{y^{\prime}}(w)
\end{array}
$$

$$
g_{y^{\prime}}(w) \geq U^{A u t}\left(y^{\prime}\right) \quad \forall y^{\prime} \in Y
$$

eliminating the dependence of $V$ on $y$.

Existence of optimal allocation rules for a given intertemporal price $R$.

We will first prove existence of optimal allocation rules in a problem with additional constraints. We will then characterize the solution of this problem and we will show that the additional constraints will not be binding so that the solution to the problem with additional constraints is also solution to the original problem.

The modified Bellman equation will be defined on $C(A)$, that is the space of continuous and bounded functions on $A$ where $A=\{w \in \Re \mid \underline{w} \leq w \leq \bar{w}\} \subseteq D$ is a compact subset of $\Re$ and $\underline{w}:=\min _{y} U^{A u t}(y)$. Consider the operator $T_{R}$ defined as:

$$
\begin{aligned}
T_{R} V(w)= & \min _{h, g_{y^{\prime}}}\left\{\left(1-\frac{1}{R}\right) C(h)+\frac{1}{R} \sum_{y^{\prime} \in Y} \pi\left(y^{\prime}\right) V\left(g_{y^{\prime}}\right)\right\} \\
& \text { s.t } \\
w= & (1-\beta) h+\beta \sum_{y^{\prime} \in Y} \pi\left(y^{\prime}\right) g_{y^{\prime}} \\
U^{A u t}\left(y^{\prime}\right) \leq & g_{y^{\prime}} \leq \bar{w} \quad \forall y^{\prime} \in Y
\end{aligned}
$$

Notice that the inequalities $g_{y^{\prime}} \leq \bar{w}$ in $(21)$ are what distinguish this modified problem 
from the original problem. Also note that (21) and (20) imply that for every $w$ in $A$

(22) $\underline{h}(w) \equiv \frac{w-\beta \bar{w}}{(1-\beta)} \leq h \leq \frac{w-\beta \sum \pi\left(y^{\prime}\right) U^{A u t}\left(y^{\prime}\right)}{(1-\beta)} \equiv \bar{h}(w)$

This in turn implies that $\bar{w}$ must be chosen such that $\underline{h}(w)$ and $\bar{h}(w)$ are in $D$ for every $w$. We will show below that we can choose $\bar{w}=\max _{y} U^{A u t}(y)+\varepsilon$, for $\varepsilon>0$ arbitrarily small. Then under the following assumption 3, (22) is always satisfied.

Assumption 3: Define $\underline{h}=\frac{\underline{w}-\beta \max _{y} U^{A u t}(y)}{1-\beta}$ and $\bar{h}=\frac{\max _{y} U^{A u t}(y)-\beta \sum \pi(y) U^{A u t}(y)}{1-\beta}$. We assume that $(\underline{h}, \bar{h}) \subseteq D$.

Note that this is an assumption purely on the fundamentals of the economy. With the additional constraint we obtain a bounded dynamic programming problem. We can then go on to characterize the operator $T_{R}$ as a contraction mapping, using Blackwell's sufficient conditions.

Lemma 1. $T_{R}$ maps $C(A)$ into itself and is a contraction.

Proof. See Appendix

Corollary 1. For $R>1$, the operator $T_{R}$ has a unique fixed point $V_{R} \in C(A)$ (i.e. $V_{R}$ is continuous and bounded) and for all $v_{0} \in C(A),\left\|T_{R}^{n} v_{0}-V_{R}\right\| \leq \frac{1}{R^{n}}\left\|v_{0}-V_{R}\right\|$, with the norm being the sup-norm.

Characterization of Value and Policy Functions We now proceed to characterize the unique fixed point of $T_{R}, V_{R}$. The fixed point to the functional equation basically inherits all properties of the period cost function $C$ in that it is strictly increasing and has increasing marginal costs.

Lemma $2 . V_{R}$ is strictly increasing and strictly convex.

\section{Proof. See Appendix}

In the next lemma we characterize the properties of the operator $T_{R}$ further. This will be useful in deducing further properties of the fixed point $V_{R}$. 
LEMma 3. For any strictly increasing and strictly convex function $V \in C(A), T_{R} V$ is continuous, strictly increasing and strictly convex. The optimal policies $h(w), g_{y^{\prime}}(w)$ are continuous, single-valued functions.

\section{Proof. See Appendix}

We will use the first order conditions heavily to characterize optimal policies. For this we first have to establish that the value function is differentiable. For any convex and differentiable function $V \in C(A)$ and fixed $w \in A$ the first order conditions characterizing the optimal choices of $h=h(w)$ and $g_{y^{\prime}}=g_{y^{\prime}}(w)$ for the problem (19) are:

$$
\begin{aligned}
C^{\prime}(h) & \leq \frac{1-\beta}{\beta(R-1)} V^{\prime}\left(g_{y^{\prime}}\right) \\
& =\frac{1-\beta}{\beta(R-1)} V^{\prime}\left(g_{y^{\prime}}\right) \quad \text { if } \quad g_{y^{\prime}}>U^{A u t}\left(y^{\prime}\right) \\
w & =(1-\beta) h+\beta \sum_{y^{\prime} \in Y} \pi\left(y^{\prime}\right) g_{y^{\prime}}
\end{aligned}
$$

The envelope condition is:

$$
\left(T^{R} V\right)^{\prime}(w)=\frac{(R-1)}{R(1-\beta)} C^{\prime}(h)
$$

We now prove that we in fact can use these conditions to characterize the optimal policies by showing that the solution to the functional equation is differentiable.

Lemma 4. The unique fixed point of $T_{R}$ is continuously differentiable.

\section{Proof. See Appendix}

We now turn to the characterization of the optimal policies. First we characterize the behavior of $h$ and $g_{y^{\prime}}$ with respect to $w$. It turns out that the social planner reacts to a higher utility promise by increasing current and expected future utility, i.e.. by smoothing the cost as much as possible over time and across states. The only constraint that prevents complete cost smoothing over different states is the continuing participation constraint: certain agents have to be promised more than otherwise optimal in certain states to be prevented from reverting to the autarkic allocation in that state. This is exactly the reason why complete risk sharing may not be constrained feasible in this environment. 
Lemma 5. Suppose $V \in C(A)$ is strictly convex and differentiable. Then the optimal policy $h$, associated with the minimization problem in (19) is strictly increasing in $w$.

Proof. See Appendix

Lemma 6. Suppose $V \in C(A)$ is strictly convex and differentiable. Then the optimal policies $g_{y^{\prime}}$, associated with the minimization problem in 11 are constant in $w$ and equal to $U^{A u t}\left(y^{\prime}\right)$ or strictly increasing in $w$, for all $y^{\prime} \in Y$.

Proof. See Appendix

Lemma 7. Suppose $V \in C(A)$ is strictly convex and differentiable.

$$
\begin{aligned}
& \text { If } g_{y^{\prime}}(w)>U^{A u t}\left(y^{\prime}\right) \text { and } g_{\bar{y}^{\prime}}(w)>U^{A u t}\left(\bar{y}^{\prime}\right) \text {, then } g_{y^{\prime}}(w)=g_{\bar{y}^{\prime}}(w) . \\
& \text { If } g_{y^{\prime}}(w)>U^{A u t}\left(y^{\prime}\right) \text { and } g_{\bar{y}^{\prime}}(w)=U^{A u t}\left(\bar{y}^{\prime}\right) \text {, then } g_{y^{\prime}}(w) \leq g_{\bar{y}^{\prime}}(w) \text { and } y^{\prime}<\bar{y}^{\prime}
\end{aligned}
$$

Proof. Follows directly from the first order condition

The last lemma states that future promises are equalized across states whenever the continuing participation constraints permit it. Promises are increased in those states in which the constraints bind.

Now we state a result that is central for the existence of an upper bound $\bar{w}$ of utility promises. For promises that are sufficiently high it is optimal to deliver most of it in terms of current period utility, and promise less for the future than the current promises. This, in effect, puts an upper bound on optimal promises in the long run. This is the content of the main result in this section, stated in Theorem 2.

LEMMA 8. Let $\left\{g_{y^{\prime}}\right\}_{y^{\prime} \in Y}$ be the optimal policies associated with the unique fixed point of $T_{R}, V_{R}$. For every $w \in A$ and every $y^{\prime} \in Y$, if $g_{y^{\prime}}(w)>U^{A u t}\left(y^{\prime}\right)$, then $g_{y^{\prime}}(w)<w$. Furthermore, for each $y^{\prime}$, there exists a unique $w_{y^{\prime}}$ such that $g_{y^{\prime}}\left(w_{y^{\prime}}\right)=w_{y^{\prime}}$ and $w_{y^{\prime}}=U^{A u t}\left(y^{\prime}\right)$.

Proof. See Appendix

THEOREM 2. There exists a $\bar{w}$ such that $g_{y^{\prime}}(w)<w$ for every $w \geq \bar{w}$ and every $y^{\prime} \in Y$.

Proof. See Appendix 
Note that the preceding theorem implies that whenever $w \in[\underline{w}, \bar{w}]$, then for all $y^{\prime} \in Y$, the constraint $g_{y^{\prime}}(w) \leq \bar{w}$ is never binding and assumption 3 guarantees that $h(w) \in D$. We will analyze the Markov process on $A \times Y$ induced by the exogenous $\pi$ and the endogenous $g_{y^{\prime}}$. Before this, however, we discuss the relationship between the component planning problem and the solution to the functional equation.

\section{Equivalence between the CPP in sequential and recursive formulation}

In this section we show that the allocation $\hat{\sigma}=\left\{\hat{h}_{t}\left(w_{0}, y^{t}\right)\right\}_{t=0}^{\infty}$ induced by the optimal policies from the recursive formulation, $h(w), g_{y^{\prime}}(w)$ solve the $C P P$ in sequential formulation, i.e. solve the program CPP for interest rates $\left\{R_{t}\right\}_{t=0}^{\infty}$ constant at $R$. So for given $\left(w_{0}, y_{0}\right)$ let $\hat{h}_{0}\left(w_{0}, y_{0}\right)=h\left(w_{0}\right), \hat{w}_{1}\left(w_{0}, y_{0}\right)=g_{y_{1}}\left(w_{0}\right)$ and in general $\hat{w}_{t}\left(w_{0}, y^{t}\right)=g_{y_{t}}\left(\hat{w}_{t-1}\left(w_{0}, y^{t-1}\right)\right)$ and $\hat{h}_{t}\left(w_{0}, y^{t}\right)=h\left(\hat{w}_{t}\left(w_{0}, y^{t}\right)\right)$ be the allocation induced by the recursive policy rules. In the appendix we invoke the principle of optimality to argue that the allocation so derived solves CPP for constant interest rates.

TheOREM 3. Suppose that the sequence $\left\{R_{t}\right\}_{t=0}^{\infty}$ is constant at $R \in\left(1, \frac{1}{\beta}\right)$. Then the allocation $\hat{\sigma}$ constructed from the optimal policies of the functional equation solves the component planning problem, for every $\left(w_{0}, y_{0}\right) \in W \times Y$ with $w_{0} \geq U^{A u t}\left(y_{0}\right)$.

Proof. See Appendix

\section{Existence and uniqueness of an invariant probability measure}

Let $W=[\underline{w}, \bar{w}]$ and $\mathcal{B}(W), \mathcal{P}(Y)$ the set of Borel sets of $W$ and the power set of $Y$. The function $g_{y^{\prime}}(w)$, together with the transition function $\pi$ for the endowment process, defines a Markov transition function on income shock realizations and utility promises $Q$ : $(W \times Y) \times(\mathcal{B}(W) \times \mathcal{P}(Y)) \rightarrow[0,1]$ as follows:

$$
Q(w, y, \mathcal{W}, \mathcal{Y})=\sum_{y^{\prime} \in \mathcal{Y}}\left\{\begin{array}{l}
\pi\left(y^{\prime}\right) \quad \text { if } \quad g_{y^{\prime}}(w) \in \mathcal{W} \\
0 \quad \text { else }
\end{array}\right.
$$


Given this transition function, we define the operator $T^{*}$ on the space of probability measures $\Lambda((W \times Y),(\mathcal{B}(W) \times \mathcal{P}(Y))$ as

$$
\left(T^{*} \lambda\right)(\mathcal{W}, \mathcal{Y})=\int Q(w, y, \mathcal{W}, \mathcal{Y}) d \lambda=\sum_{y^{\prime} \in \mathcal{Y}} \pi\left(y^{\prime}\right) \int_{\left\{w \in W \mid g_{\mathcal{y}^{\prime}}(w) \in \mathcal{W}\right\}} d \lambda
$$

for all $(\mathcal{W}, \mathcal{Y}) \in \mathcal{B}(W) \times \mathcal{P}(Y)$. Note that $T^{*}$ maps $\Lambda$ into itself (see Stokey et. al. (1989), Theorem 8.2). An invariant probability measure associated with $Q$ is defined to be a fixed point of $T^{*}$. In this section we address the question of whether such a probability measure exists and is unique. Intuitively, this invariant measure describes the long-run implications of the planners cost minimizing policies.

We first add a property of $g_{y^{\prime}}$ that will be useful in proving the existence of a unique invariant probability measure associated with the transition function $Q$.

Lemma 9. There exists $w^{*} \in A$ such that $w^{*}>U^{A u t}\left(y_{\max }\right)$ and $g_{y_{\max }}\left(w^{*}\right)=U^{A u t}\left(y_{\max }\right)$

Proof. See Appendix

Corollary 2. $g_{y_{\max }}\left(w^{*}\right)=U^{\text {Aut }}(y)$ for all $w \leq w^{*}$.

With this result and the characterization of the functions $g_{y^{\prime}}$ from previous sections we can prove the main result of this section, namely that for a given $R \in\left(1, \frac{1}{\beta}\right)$, there is a unique invariant measure over utility promises and endowment shocks associated with transition function $Q$.

THEOREM 4. There exists a unique invariant probability measure $\Phi$ associated with the transition function $Q$ defined above and for all $\Phi_{0} \in \Lambda\left((W \times Y),(\mathcal{B}(W) \times \mathcal{P}(Y)),\left(T^{*} \Phi_{0}\right)^{n}\right.$ converges to $\Phi$ in total variation norm.

\section{Proof. See Appendix}

REMARK 3. The argument above also shows that any ergodic set of the Markov process associated with $Q$ must lie within $\left[U^{A u t}\left(y_{\min }\right), U^{A u t}\left(y_{\max }\right)\right] \times Y$ and that the support of the unique invariant probability measure is a subset of this set. For any initial measure over 
shocks and utility entitlements, in the long run the planners' actions result in the invariant cross-sectional measure.

This concludes the discussion of the Markov process on utility promises for a fixed interest rate $R \in\left(1, \frac{1}{\beta}\right)$. In our subsequent analysis we will index policy functions $\left(h, g_{y^{\prime}}\right)$, cost functions $V$ and unique invariant probability measures $\Phi$ by $R$ to make clear that these functions and measures were derived for a fixed $R$. So far we have not imposed any resource feasibility condition. It may be the case that for a fixed $R$, in order to deliver a distribution over utility entitlements $\Phi_{R}$, more resources than available have to be used up. In the next section we will analyze how the resource requirements imposed by $\Phi_{R}$ vary with $R$.

\section{Determination of the "market clearing" shadow price $R$}

In the previous section we showed that for a fixed $R \in\left(1, \frac{1}{\beta}\right)$ there exists a unique stationary joint distribution over $(w, y)$. Define the "excess demand function" associated with $R$ as

$$
d(R)=\int V_{R}(w) d \Phi_{R}-\int y d \Phi_{R}
$$

In this section we analyze the qualitative features of the function $d($.$) . Since by assumption$ $\int y d \Phi_{R}$ does not vary with $R$, the behavior of $d$ depends on how $V_{R}$ and $\Phi_{R}$ vary with $R$. The behavior of $\Phi_{R}$ with respect to $R$ in turn depends on the behavior of $g_{y^{\prime}}^{R}$ with respect to $R$ as $g_{y^{\prime}}^{R}$ determines the Markov process to which $\Phi_{R}$ is the invariant probability measure. We start by proving that the function $V^{R}$ varies continuously with $R$, then show that the optimal policies $g_{y^{\prime}}^{R}$ vary continuously in $R$. These results, in turn, imply continuity of $d$, as shown in Theorem 5 .

LEMma 10. (Atkeson and Lucas (1995)) Let $R \in\left(1, \frac{1}{\beta}\right)$ and $\left\{R_{n}\right\}_{n=0}^{\infty}$ be a sequence satisfying $R_{n} \in\left(1, \frac{1}{\beta}\right)$ and $\lim _{n \rightarrow \infty} R_{n}=R$. Then the sequence $\left\{V_{R_{n}}\right\}_{n=0}^{\infty}$ converges uniformly to $V_{R}$ on $[\underline{w}, \bar{w}]$.

Proof. See Appendix 
LEMma 11. (Atkeson and Lucas (1995)) Let a sequence $\left\{R_{n}, w_{n}\right\}_{n=0}^{\infty}$ with $R_{n} \in\left(1, \frac{1}{\beta}\right)$ and $w_{n} \in[\underline{w}, \bar{w}]$ converge to $(R, w) \in\left(1, \frac{1}{\beta}\right) \times[\underline{w}, \bar{w}]$. Then for each $y^{\prime} \in Y$, the sequence $\left\{g_{y^{\prime}}^{R_{n}}\left(w_{n}\right)\right\}_{n=0}^{\infty}$ converges to $g_{y^{\prime}}^{R}(w)$.

\section{Proof. See Appendix}

The previous two lemmas can be used to prove our first main result about the excess demand function $d($.$) , namely continuity on \left(1, \frac{1}{\beta}\right)$.

TheOrem 5. (Atkeson and Lucas (1995), Lemma 12): $d(R)$ is continuous on $\left(1, \frac{1}{\beta}\right)$.

\section{Proof. See Appendix}

The previous result establishes that the excess demand function varies continuously with $R$. Now we want to establish a result about the slope of the excess demand function. Intuitively, a higher interest rate $R$ makes resources tomorrow cheaper compared to resources today. This should lead, for given utility entitlement $w$, to a decrease in utility received today and an increased promised utility from tomorrow onwards. Since the invariant measure over utility is determined by future utility promises, for higher $R$ one expects higher utility promises on average, and hence higher resource costs. In other words, one expects the excess demand function to be increasing in $R$.

In order to prove this we first establish that future utility promises are indeed increasing in the interest rate $R$.

LEMma 12. The optimal policies $g_{y^{\prime}}^{R}(w)$ are increasing in $R$ and the optimal policy $h^{R}(w)$ is decreasing in $R$.

\section{Proof. See Appendix}

This result enables us to draw conclusions about how the invariant measure over utilities and endowment shocks, $\Phi_{R}$ varies with $R$. The next result shows that for larger interest rates the invariant measure puts more mass on higher utility entitlements. For every $\Phi$ define the probability measures $\Phi^{y}$ on $(W, \mathcal{B}(W))$ by $\Phi^{y}(B)=\frac{\Phi(B,\{y\})}{\pi(y)}$, for every $B \in \mathcal{B}(W)$. Note that every such measure is well-defined as $\pi(y)>0$ by assumption, and that $\Phi^{y}(W)=1$. 
LEMma 13. (Atkeson and Lucas (1995)) Let $R>\hat{R}$. Then for every $y \in Y, \Phi_{R}^{y}$ stochastically dominates $\Phi_{\hat{R}}^{y}$, i.e.. for every increasing and continuous function $f$ on $W$,

(28) $\int f(w) d \Phi_{R}^{y} \geq \int f(w) d \Phi_{\hat{R}}^{y}$

Proof. See Appendix

The previous results can be combined to show that the excess demand function is increasing in the interest rate.

Theorem 6. (Atkeson and Lucas (1995), Lemma 14) Let $R>\hat{R}$. Then $d(R) \geq d(\hat{R})$.

Proof. See Appendix

The previous results show that the excess demand function is continuous and increasing on $\left(1, \frac{1}{\beta}\right)$. The behavior of the excess resource function at $R=\frac{1}{\beta}$ (the complete markets shadow price) is easily determined. For such $R, g_{y^{\prime}}(w)=w$ or $g_{y^{\prime}}(w)=U^{A u t}\left(y^{\prime}\right)$ from the first order conditions. There is a continuum of invariant measures. No $w<g_{y^{\prime}}\left(U^{A u t}\left(y_{\max }\right)\right)$ is in the support of any of these measures, though, as the probability of leaving such a $w$ is at least $\pi\left(y_{\max }\right)$ and the probability of coming back (into a neighborhood) is 0 . Therefore $g_{y^{\prime}}(w)=w$ for all points in the support of the invariant measure, and there is complete risk sharing as from the promise-keeping constraint $h(w)=w$. Each individuals' consumption is constant over time. The invariant measure with lowest cost is the one that puts mass $\pi(y)$ on $\left(U^{A u t}\left(y_{\max }\right), y\right)$. Hence each individual receives the same current utility, hence the same current consumption, which is equal to the average after-tax endowment $\bar{y}$. For this allocation to be incentive feasible one needs

$$
U^{A u t}\left(y_{\max }\right) \leq u(\bar{y})
$$

Since we are interested in efficient allocations that don't feature perfect risk sharing we assume that the above inequality is violated. ${ }^{7}$ Then complete risk sharing is not possible with resources $\bar{y}$. By arguments similar to the proof of continuity one can show that for

\footnotetext{
${ }^{7}$ The inequality will be violated for $\beta$ sufficiently small and/or the income process sufficiently variable.
} 
$R \rightarrow \frac{1}{\beta}$, the cost of the associated allocation converges to the cheapest complete risk sharing allocation. Hence under the assumption made $\lim _{R \rightarrow \frac{1}{\beta}} d(R)>0$.

On the other hand, for $R$ close to 1 , there are two possible situations. If

$$
\beta \frac{u^{\prime}\left(y_{\min }\right)}{u^{\prime}\left(y_{\max }\right)}<1
$$

then the autarkic allocation satisfies the first order conditions for some $R>1$. Since autarky is constrained feasible, it is efficient. If the inequality in (29) is reversed, we conjecture that a) autarky is not efficient and b) $d(R)<0$ for $R$ sufficiently close to, but bigger than $1 .^{8}$

We assume that 29 does not hold. ${ }^{9}$ Then under the last two assumptions, there exists an efficient stationary allocation different from complete risk sharing and different from the autarkic solution. ${ }^{10}$

REMARK 4. The previous discussion is valid for any exogenously given interest rate on one period bonds, $r$. If one requires this interest rate to be equal to the implicit interest rate for risk sharing contracts, i.e. $1+r=R$, as we do in our quantitative analysis, then $U^{A u t}(y ; r)=$ $U^{\text {Aut }}(y ; R-1)$ varies in $R$. All theoretical results apart from those in section Dgo through unchanged. Lemma 10 and 11 go through as the operator $T_{R}$ is still continuous in $R$. Therefore theorem 5 is still valid and the excess demand function is still continuous. However it need not be monotonically increasing and hence the set of equilibrium $R^{\prime} s$ need not be convex. The discussion about the behavior of $d(R)$ at $R=\frac{1}{\beta}$ and close to 1 also remain valid.

\section{Qualitative Features of the Efficient Allocation}

In figure 1 (see the appendix) we show the typical shape of the policy functions $g_{y^{\prime}}(w)$. The policies shown here are computed a specific parameterization of the model, in which the endowment process can take on two values, $y_{l}<y_{h}$ and a specific interest rate $R \in\left(1, \frac{1}{\beta}\right)$. We observe the following properties: as shown above $g_{y^{\prime}}(w)$ is greater or equal to $U^{A u t}\left(y^{\prime}\right)$,

\footnotetext{
${ }^{8}$ For the case that the endowment process can take only two values and utility is of CRRA-form we were able to prove this conjecture, but not for the general case considered here.

${ }^{9}$ As is well known, for sufficiently low $\beta$ only the autarkic allocation satisfies the continuing participation constraints. See Alvarez and Jermann (1998a) for a discussion of the two-agent case.

${ }^{10}$ No claim of uniqueness can be made at this point. The set of efficient shadow prices $R$ is convex. In our computational exercises, however, uniqueness always arises.
} 
constant at $U^{A u t}\left(y^{\prime}\right)$ for $w \leq U^{A u t}\left(y^{\prime}\right)$ and intersects the $45^{0}$-line at $w=U^{A u t}\left(y^{\prime}\right)$. For $w>U^{A u t}\left(y^{\prime}\right)$ is either constant at $U^{A u t}\left(y^{\prime}\right)$ or strictly increasing in $w$, and lies below the $45^{0}$-line. Furthermore $g_{y_{l}}(w) \leq g_{y_{h}}(w)$.

The support of the invariant measure $\Phi$ with respect to $w$ is, as shown above, equal to $\left[U^{A u t}\left(y_{l}\right), U^{A u t}\left(y_{h}\right)\right]$. The features of the policy functions just described imply that an agent with $y^{\prime}=y_{h}$ has $g_{y^{\prime}}(w)=U^{A u t}\left(y_{h}\right)$ and hence the highest possible future utility entitlements in the support of $\Phi$, regardless of her $w$. Hence, when an agent receives a high income shock, history is erased in that present utility entitlements $w$, which summarize the history of past endowment shocks, do not matter for future utility entitlements. For agents with $y^{\prime}=y_{l}$, history matters. An agent with $w=U^{A u t}\left(y_{h}\right)$ that receives $y^{\prime}=y_{l}$ drops to $g_{y^{\prime}}\left(U^{A u t}\left(y_{h}\right)\right)$ $<U^{A u t}\left(y_{h}\right)$, and, upon further bad shocks, works herself downwards through the entitlement distribution. In finite (if $g_{y_{l}}(w)=U^{A u t}\left(y_{l}\right)$ for some $w>U^{A u t}\left(y_{l}\right)$ ) or infinite number of steps an agent with a string of bad shocks arrives at $w=U^{A u t}\left(y_{l}\right)$, with any good shock putting her immediately back to $U^{A u t}\left(y_{h}\right)$. Note from the theoretical results that the features of immediate jumping up upon receiving the highest shock as well as gradual working down upon receiving lower shocks hold for an arbitrary (finite) number of states for the endowment process.

The properties of the entitlement distribution immediately translate into properties of the stationary consumption measure as the latter is given as

$$
\Psi(A)=\int_{\{w \mid C(h(w)) \in A\}} d \Phi
$$

Since $h($.$) and C($.$) are strictly increasing functions, consumption reacts to endowment shocks$ in exactly the same qualitative fashion as utility entitlements. This can be seen from figure 2. All agents with a high endowment shock consume the same, regardless of their history and consume the maximum amount in the support of the consumption distribution. Hence the consumption distribution has a mass point at this consumption level equal to $\Pi\left(y_{h}\right)$. Another mass point occurs at the minimum of the support of the consumption distribution, provided that $g_{y_{l}}(w)=U^{A u t}\left(y_{l}\right)$ for some $w>U^{A u t}\left(y_{l}\right)$ (which is true in the present example, but need not be true in general). All agents that have a sufficiently long string of bad income 
realizations will consume the minimum consumption level in the support. In between these two mass points there is a finite number of other mass points consisting of individuals with currently bad shock who are in the process of either falling to the bottom (with additional bad shocks) or jumping again to the top (with a single good shock), but who haven't hit rock bottom yet.

What is the economic intuition for these results? One observes that those individuals that experience an increase in income from today to tomorrow are constrained. This can be interpreted as follows. These agents have borrowed against the chance of being lucky in the next period (in utility terms, as they receive more utility today than in autarky) and if the contingency of being lucky tomorrow materializes they are called upon to repay their debt and transfer resources to other agents. Unconstrained agents tend to be those with a drop in income. These agents are unconstrained tomorrow as they will receive transfers (from agents with favorable income shock tomorrow) at that date, which makes defaulting unreasonable. This feature of the model justifies the term "debt constrained" as agents are constrained to borrow today because of their possible incentive to default if called upon to pay back tomorrow (in the contingency of a high income realization).

These results are only suggestive for an economy with more than 2 shocks. However, the phenomena of jumping to the top in one step upon receiving the high endowment shocks and stepwise working through the distribution with consistently low endowment shocks is an inherent property of the model. For higher numbers of exogenous states, of course, a richer consumption distribution "in the middle" arises.

Finally, in figure 3 we show the excess demand function $d(R)$ for a fixed parameterization of the model. As proved in the theoretical part, this function is continuous and increasing in $R$ on $\left(1, \frac{1}{\beta}\right)$. In the specific example it has a unique $R^{*} \in\left(1, \frac{1}{\beta}\right)$ such that $d(R)$ equals to 0 .

\section{Decentralization}

In this section we turn to the question of decentralizing the efficient consumption allocation through a market mechanism. In doing so we will follow the approach of Kehoe and Levine (1993). Consider an agent with period zero endowment of $y_{0}$ and initial wealth of $a_{0}$. Wealth is measured as entitlement to the period 0 consumption good. Let $\Theta$ be the 
joint distribution over $\left(a_{0}, y_{0}\right)$.

Let denote by $p_{t}\left(y^{t}\right)$ the date zero price of a contract that specifies delivery of one unit of the consumption good at period $t$ to/from a person who has experienced endowment shock history $y^{t}$. For each contingency $c_{t}\left(a_{0}, y^{t}\right)-y_{t}$ is the net trade of individual $\left(a_{0}, y_{0}\right)$ for that contingency. In period 0 there is no idiosyncratic uncertainty, so normalize the price of the consumption good at period 0 to $1^{11}$.

A household of type $\left(a_{0}, y_{0}\right)$ chooses an allocation $\left\{c_{t}\left(a_{0}, y^{t}\right)\right\}$ to solve

$\max (1-\beta)\left(u\left(c_{t}\left(a_{0}, y_{0}\right)\right)+\sum_{t=1}^{\infty} \sum_{y^{t} \mid y_{0}} \beta^{t} \pi\left(y^{t} \mid y_{0}\right) u\left(c_{t}\left(a_{0}, y^{t}\right)\right)\right)$

$$
\begin{aligned}
& \text { s.t. } c_{0}\left(a_{0}, y_{0}\right)+\sum_{t=1}^{\infty} \sum_{y^{t} \mid y_{0}} p_{t}\left(y^{t}\right) c_{t}\left(a_{0}, y^{t}\right) \leq a_{0}+y_{0}+\sum_{t=1}^{\infty} \sum_{y^{t} \mid y_{0}} p_{t}\left(y^{t}\right) y_{t} \\
& (1-\beta)\left(u\left(c_{t}\left(a_{0}, y^{t}\right)\right)+\sum_{s>t} \sum_{y^{s} \mid y^{t}} \beta^{s-t} \pi\left(y^{s} \mid y^{t}\right) u\left(c_{s}\left(a_{0}, y^{s}\right)\right)\right) \geq U_{t}^{A u t}\left(y_{t}\right)
\end{aligned}
$$

Note that, as in Kehoe and Levine(1993), the continuing participation constraints enter the individual consumption sets directly. ${ }^{12}$

\footnotetext{
${ }^{11}$ Note that in standard Arrow Debreu equilibrium theory with finitely many consumers, a complete description of the state of the economy would be everybody's endowment shock history, and all prices would be contingent on this complete state. With atomistic individuals, the assumed law of large number and the focus on decentralizing stationary allocations, attention can be restricted to equilibria in which prices (and quantities) depend only on own personal histories.

${ }^{12}$ The physical environment that underlies this discusion is the following: There is a central market place and a Walrasian auctioneer (or financial intermediary) that, at period 0, calls out prices for the consumption good, contingent on (perfectly observable) endowment shock histories. Based on prices individuals, at date 0 , purchase contingent consumption contracts $c_{t}\left(a_{0}, y^{t}\right)$. In each period $t$, then, individuals return to the central market place to claim their net consumption at period $t, c_{t}\left(a_{0}, y^{t}\right)-y_{t}$, which may be negative. The auctioneer acts as a clearinghouse for net claims and deliveries and enforces that agents that did not act according to the contract signed at period 0 at some past date will be excluded from the market place.
} 
Definition 3. An equilibrium consists of prices $\left\{p_{t}\left(y^{t}\right)\right\}_{t=0}^{\infty}$ and allocations $\left\{c_{t}\left(a_{0}, y^{t}\right)\right\}_{t=0}^{\infty}$ such that

- given prices, the allocation solves household's problem for almost all $\left(a_{0}, y_{0}\right)$

- markets clear, i.e. for all $t$,

$$
\int \sum_{y^{t}} c_{t}\left(a_{0}, y^{t}\right) \pi\left(y^{t} \mid y_{0}\right) d \Theta=\int \sum_{y^{t}} y_{t} \pi\left(y^{t} \mid y_{0}\right) d \Theta
$$

\section{Equilibrium Prices}

Let $\beta^{t} \pi\left(y^{t} \mid y_{0}\right) \mu\left(a_{0}, y^{t}\right) \geq 0$ be the Lagrange multiplier associated with the continuing participation constraint at history $y^{t}$ and $\lambda\left(a_{0}, y_{0}\right) \geq 0$ be the Lagrange multiplier associated with the time 0 budget constraint. Let $P\left(y^{t}\right)=\left\{y^{\tau} \mid \pi\left(y^{t} \mid y^{\tau}\right)>0\right\}$ be the set of all endowment shock histories that can have $y^{t}$ as its continuation. The first order necessary conditions are

$$
(1-\beta) \beta^{t} \pi\left(y^{t} \mid y_{0}\right) u^{\prime}\left(c_{t}\left(a_{0}, y^{t}\right)\right)\left[1+\sum_{y^{\tau} \in P\left(y^{t}\right)} \mu\left(a_{0}, y^{\tau}\right)\right]=\lambda\left(a_{0}, y_{0}\right) p\left(y^{t}\right)
$$

Combining these conditions for two consecutive time periods and histories $y^{t}, y^{t+1} \in P\left(y^{t}\right)$ we obtain

$$
\beta \frac{u^{\prime}\left(c_{t}\left(a_{0}, y^{t+1}\right)\right) \pi\left(y^{t+1} \mid y_{0}\right)}{u^{\prime}\left(c_{t}\left(a_{0}, y^{t}\right)\right) \pi\left(y^{t} \mid y_{0}\right)}=\frac{p_{t+1}\left(y^{t+1}\right)}{p_{t}\left(y^{t}\right)} \frac{1+\sum_{y^{\tau} \in P\left(y^{t}\right)} \mu\left(a_{0}, y^{\tau}\right)}{1+\sum_{y^{\tau} \in P\left(y^{t+1}\right)} \mu\left(a_{0}, y^{\tau}\right)}
$$

Obviously, an agent whose participation constraint does not bind at contingency $y^{t+1}$, following history $y^{t}$, faces the standard complete markets Euler equation (as $\left.\mu\left(a_{0}, y^{t+1}\right)=0\right)$.

Now consider the efficient allocation of utilities $\left\{h_{t}\left(w_{0}, y^{t}\right)\right\}$ as found in the previous section. Combining the first order condition and the envelope condition from the component 
planning problem we have for an agent that is unconstrained ${ }^{13}$ (see (A7)):

(35) $\frac{1}{R}=\beta \frac{C^{\prime}\left(h_{t}\left(w_{0}, y^{t}\right)\right)}{C^{\prime}\left(h_{t+1}\left(w_{0}, y^{t+1}\right)\right)}$

By definition $h_{t}\left(w_{0}, y^{t}\right)=u\left(c_{t}\left(w_{0}, y^{t}\right)\right)$ and since $C=u^{-1}$ it follows that

(36) $\frac{1}{R}=\beta \frac{u^{\prime}\left(c_{t+1}\left(w_{0}, y^{t+1}\right)\right)}{u^{\prime}\left(c_{t}\left(w_{0}, y^{t}\right)\right)}$

This suggests that the equilibrium prices satisfy

$$
p_{t+1}\left(y^{t+1}\right)=\frac{p_{t}\left(y^{t}\right) \pi\left(y^{t+1} \mid y_{0}\right)}{R \pi\left(y^{t} \mid y_{0}\right)}
$$

and from the normalization

$$
p_{t}\left(y^{t}\right)=\frac{\pi\left(y^{t} \mid y_{0}\right)}{R^{t}}=p_{t} \pi\left(y^{t} \mid y_{0}\right)
$$

with $p_{t}=R^{-t}$. That is, the price for a commodity delivered contingent on personal histories is composed of two components, an aggregate intertemporal price $p_{t}=R^{-t}$ and an individual specific, history dependent component, equal to the probability that the personal history occurs.

Given prices, the initial wealth level that makes the efficient consumption allocation affordable for an agent of type $\left(w_{0}, y_{0}\right)$ is given by

$$
a_{0}=c_{0}\left(w_{0}, y_{0}\right)-y_{0}+\sum_{t=1}^{\infty} \sum_{y^{t} \mid y_{0}} \frac{\pi\left(y^{t} \mid y_{0}\right)}{R^{t}}\left(c_{t}\left(w_{0}, y^{t}\right)-y_{t}\right)=a_{0}\left(w_{0}, y_{0}\right)<\infty
$$

where the last inequality follows from the fact that the efficient consumption allocation is bounded from above (as utility promises are bounded from above). Finally, the equilibrium

\footnotetext{
${ }^{13}$ If no agent is unconstrained we are in autarky and can take$$
\frac{1}{R}=\beta \frac{u^{\prime}\left(y_{\min }\right)}{u^{\prime}\left(y_{\max }\right)} .
$$ 
consumption allocation corresponding to the efficient allocation is given by ${ }^{14}$

$$
c_{t}\left(a_{0}, y^{t}\right)=c_{t}\left(a_{0}^{-1}\left(w_{0}, y_{0}\right), y^{t}\right)
$$

The preceding discussion can be summarized in the following theorem

THEOREM 7. Suppose that $\left\{c_{t}\left(w_{0}, y^{t}\right)\right\}_{t=0}^{\infty}$ is a stationary efficient allocation (with associated shadow interest rate $\left.R>\frac{1}{\beta}\right)$. Then prices $\left\{p_{t}\left(y^{t}\right)\right\}$ and allocations $\left\{c_{t}\left(a_{0}, y^{t}\right)\right\}$, as defined in 38 and 40 are an equilibrium for initial distribution $\Theta_{0}$ derived from $\Phi_{0}$ and 39.

Proof. See Appendix

\section{Calibration}

First we will describe how we set the parameters governing the individual endowment process, together with the government policies (spending and taxes). We will then discuss preference parameters $\beta$, the subjective time discount factor, and $\sigma$, the coefficient of relative risk aversion, as we will assume that the period utility function is of CRRA-form.

\section{Endowment process}

To characterize the Markov chain governing the individual endowment process in the model we need to set the $N$ possible values the endowment $e_{t}$ can take and estimate the transition matrix $\pi\left(e_{t+1} \mid e_{t}\right)$. In order to do so we use household level data from the Consumer Expenditure Survey (CEX) for the years 1986-1994. The main reason why we use CEX income data, whose quality is supposedly inferior to PSID data, is because CEX reports also taxes paid by the household members and transfers received, such as welfare and unemployment insurance payments. We try to reduce measurement error by excluding from our sample households ${ }^{15}$ classified as incomplete income respondents, as suggested by Nelson (1994) and

\footnotetext{
${ }^{14}$ Given that the optimal recursive policy function $h(., y)$ is a strictly increasing function in $w$, the $h_{t}\left(., y^{t}\right)$ and hence the $c_{t}\left(., y^{t}\right)$ are strictly increasing in $w_{0}$. Therefore $a_{0}\left(., y_{0}\right)$ is strictly increasing and thus invertible. We denote its inverse by $a_{0}^{-1}$.

${ }^{15}$ The CEX uses as its basic unit of investigation the Consumer Unit, which comprises either a) all members of a particular household related by blood, marriage, adoption or other legal arrangements, b) a person living alone or sharing a household with others, but is financially independent, c) two or more persons living together who use their incomes to make joint expenditure decisions. For details see Consumer Expenditure Survey (1997), p.249. We will refer to a CU as "household".
} 
Lusardi (1996). For the same reason we exclude households with zero total income (including wages, business capital income or government transfers). Finally, since we interpret our pretax endowment concept from the model as labor income we exclude households which are solely composed of members older than 64 years old. We view this as justified since our model is a model of labor income uncertainty which is completely revealed upon retirement.

The CEX quantity we will interpret as $e_{t}$, household endowment before taxes, is labor earnings. In the data we measure this entity by the sum of labor earnings, plus a fraction ${ }^{16}$ of business and farm income earned by all the members of the household, all divided by the number of adult equivalents ${ }^{17}$ in the household.

We first pick $N$ to be equal to 5 . The transition matrix $\pi\left(e_{t+1} \mid e_{t}\right)$ is computed as follows. For any quarter $t$ in the CEX sample we group households into 5 relative endowment classes delimited by 4 equally spaced quintiles $q_{.2, t}, q_{.4, t}, q_{.6, t}, q_{8, t, t}$. We then search for all households for which we have endowment observations at $t$ and $t+4\left(e_{t}\right.$ and $\left.e_{t+4}\right)$ and compute in which relative class they belong in period $t+4$. Notice that here the class delimiters depend upon time to take account of aggregate growth. We repeat this for every quarter in the sample. Then the probability of transiting from class $i$ to class $j$ is given by the number of households transiting from $i$ to $j$, divided by the total number of households starting in class $i$ for the entire sample 1986-1994.

Once $\pi\left(e_{t+1} \mid e_{t}\right)$ is computed we set $e_{1, . .}, e_{N}$ to be equal to the median income for each endowment class in $1994 .{ }^{18}$ Table 1 and 2 show the results of this exercise. Note that the stationary distribution associated with the transition matrix is given by $\Pi=$ $[0.18,0.21,0.21,0.2,0.2]$. For our quantitative exercises we normalize the endowment values $e_{1, . .}, e_{N}$ so that the aggregate pre-tax endowment $\sum_{j=1}^{5} \Pi\left(e_{j}\right) e_{j}=1$.

\footnotetext{
${ }^{16}$ The fraction of business and farm income we impute to labor income is .864 as reported in Diaz Jimenez, Quadrini, Rios Rull (1997).

${ }^{17}$ The number of adult equivalent is defined as in Deaton and Paxon (1994) as the number of households mebres over age 16 plus .5 times the number of members below age 16 .

${ }^{18}$ We take the average of the medians of the four quarters in 1994.
} 
Table 1. Endowment values (Ratio to 1994 median)

\begin{tabular}{l|l|l|l|l}
$e_{1}$ & $e_{2}$ & $e_{3}$ & $e_{4}$ & $e_{5}$ \\
\hline \hline .21 & .62 & 1 & 1.49 & 2.53
\end{tabular}

Table 2. Transition Matrix (1986.1-1994.4), (26747 obs.)

\begin{tabular}{c||ccccc}
\multicolumn{1}{c||}{} & \multicolumn{5}{c}{ Quintile at $t+4$} \\
\cline { 2 - 6 } Quintile at $t$ & 1 & 2 & 3 & 4 & 5 \\
\hline \hline 1 & .68 & .21 & .06 & .03 & .02 \\
2 & .18 & .56 & .19 & .05 & .02 \\
3 & .05 & .18 & .55 & .19 & .04 \\
4 & .03 & .05 & .19 & .60 & .14 \\
5 & .02 & .02 & .03 & .15 & .78
\end{tabular}

\section{Fiscal policy}

To characterize the fiscal policy we need to measure the values of $\tau\left(e_{j}\right), j=1, \ldots, N$. In CEX households are asked to report federal state and local taxes deducted from their last paycheck separately from any additional (not deducted form paycheck) federal state and local taxes paid. Since we want a measure of taxes on labor earnings the first measure seems more appropriate for our purposes. We add to taxes social security contributions and subtract transfers (welfare, unemployment compensation and food stamps). We then set $\tau\left(e_{j}\right)$ equal to the ratio between the total sum of federal state and local taxes and social security deducted from paycheck, net of transfers, in the $j$-th earnings class and the total labor income as measured above in the same class. Once the tax policy is set we can compute the implied level of government spending (net of transfers) such that the budget is balanced in every period. The tax policy we will use in our experiments is the average of the tax policies measured in the four quarters of 1994 and is reported below. 
Table 3. Tax Rates, 1994, ( 8679 obs.)

\begin{tabular}{c||ccccc}
\multicolumn{1}{c||}{} & \multicolumn{5}{c}{ Quintiles } \\
\cline { 2 - 6 } & 1 & 2 & 3 & 4 & 5 \\
\hline \hline Average Tax Rates (\%) & -35.8 & 8.1 & 15.0 & 17.3 & 20.9
\end{tabular}

The government spending (net of transfers) implied by these tax rates is equal to $g=15.8 \%$ of total endowment.

\section{Preference parameters}

We calibrate the preference parameters $(\sigma, \beta)$ so that the solution for the benchmark model delivers an interest rate of $2.5 \%$ per year. This was the average real return on AAAgrade municipal bonds (with 10 year maturity) over the period 1986-1994. We use the interest rate on municipal bonds since returns on these bonds are usually tax-exempt and hence marginal tax rates for interest income need not be specified.

In particular we set $\sigma$ equal to 2 and then choose $\beta$ so to match the interest rate. The non-standard part of this exercise is that in a debt constrained economy for a given $\sigma$ there might be multiple $\beta$ that deliver the same interest rate. In figures 4 and 5 we show the relation between the time discount factor and the interest rate in our economy and in two other economies. To understand the nonmonotonic behavior of the relation in the economy with debt constraints remember that the real interest rate is given by the marginal rate of substitution of an unconstrained agent, that is

$$
\frac{1}{R}=\beta \max _{w_{0}, y^{t+1}} \frac{u^{\prime}\left(c_{t+1}\left(w_{0}, y^{t+1}\right)\right.}{u^{\prime}\left(c_{t}\left(w_{0}, y^{t}\right)\right)}
$$

There are two critical values of the time discount factor $\beta$. If $\beta>\beta^{C M}=.99$ then the efficient allocation involves complete risk sharing of idiosyncratic risk, individual consumption is constant, $\frac{u^{\prime}\left(c_{t+1}\left(w_{0}, y^{t+1}\right)\right.}{u^{\prime}\left(c_{t}\left(w_{0}, y^{t}\right)\right)}$ is always equal to 1 and the gross interest rate $R$ equals $\frac{1}{\beta}$, hence is decreasing in $\beta$. If $\beta<\beta^{A u t}=.0207$ then autarky is an efficient allocation, $\frac{1}{R}=\beta \frac{u^{\prime}\left(y_{\min }\right)}{u^{\prime}\left(y_{\max }\right)}$ (as argued is section $\$$. and again the interest rate is decreasing in $\beta$. For $\beta \in\left(\beta^{A u t}, \beta^{C M}\right.$ ) there is some, but not complete risk sharing. As $\beta$ moves from $\beta^{C M}$ to $\beta^{A u t}$ there are two effects on the interest rate: there is a direct effect due to the decrease of $\beta$ that raises 
the interest rate and there is an indirect effect: lower $\beta$ reduces possible risk sharing ${ }^{19}$ and therefore $\max _{w_{0}, y^{t+1}} \frac{u^{\prime}\left(c_{t+1}\left(w_{0}, y^{t+1}\right)\right.}{u^{\prime}\left(c_{t}\left(w_{0}, y^{t}\right)\right)}$ tends to increase with a reduction in $\beta$, and the interest rate is reduced. As we can see from figures $4-5$, when $\beta$ is close to $\beta^{C M}$ the first effect dominates and when $\beta$ is close to $\beta^{A u t}$ the second effect dominates. From figure 4 we see that for a fixed value of $\sigma=2$ there are three possible values of $\beta(\beta=.0202, \beta=.0212, \beta=.972)$ consistent with an interest rate of $2.5 \%$. We will discuss the arising allocations for all three cases in the next section.

We summarize the parameter values for our benchmark economy in the table 4

Table 4. Preference Parameters.

\begin{tabular}{l|l|l}
\multicolumn{2}{l|}{ Parameter } & Value \\
\hline \hline$\beta$ & Time Discount Factors & $\{.972, .0212, .0202\}$ \\
$\sigma$ & Risk Aversion & 2
\end{tabular}

\section{Measures of Intermediation of Risk}

In this economy there are two ways in which individual consumption can be isolated from random fluctuations in pre-tax income. Via the tax system the government can reduce the variance in after-tax income. Private markets then serve to isolate private consumption from fluctuations in after-tax income. We measure the strength of both risk sharing means in the following way. We define as Total Intermediation (TI) of risk the ratio of cross-sectional consumption variability to pre-tax income variability, or more precisely, as one minus the ratio between the standard deviations of log-consumption to log-pre-tax income ${ }^{20}$

$$
T I=1-\frac{s t d(\log (c))}{s t d(\log (e))}
$$

Note that when $\operatorname{std}(\log (c))=0, T I=1$; consumption does not vary at all across individuals and the economy exhibits complete risk sharing. If $\operatorname{std}(\log (c))=\operatorname{std}(\log (e)), T I=0$ and consumption varies as strongly as pre-tax endowments. This is the case if individuals live in

\footnotetext{
${ }^{19}$ Households with high income realizations discount the future possibility of having low income very heavily and therefore large transfers from these agents to low-income agents violate the continuing participation constraints for these agents.

${ }^{20}$ We take logs to make the standard deviations independent of the units of measurement.
} 
complete autarky, without access even to publicly administered risk sharing. For $0<T I<1$ there is some, but not complete risk sharing, with higher TI indicating higher risk sharing.

We can decompose TI into two components reflecting risk intermediation enforced by the government $(G I)$ via the tax system and risk intermediation achieved in addition by private insurance contracts, $(P I)$. In particular we define

$$
G I=1-\frac{s t d(\log (y))}{s t d(\log (e))}
$$

and

$$
P I=1-\frac{s t d(\log (c))}{s t d(\log (y))}
$$

When $\operatorname{std}(\log (y))=\operatorname{std}(\log (e)), G I=0$. After-tax income is as variable as pre-tax income, which is the case if the tax system is proportional. If $\operatorname{std}(\log (y))<\operatorname{std}(\log (e)), G I>0$ and the tax system is progressive, with the extreme of complete redistribution via the tax system, $\operatorname{std}(\log (y))=0$ and $G I=1$. On the other hand, if $\operatorname{std}(\log (y))>\operatorname{std}(\log (e)), G I<0$ and the tax system is regressive. The interpretation of $G I$ is similar: if $\operatorname{std}(\log (c))=0$, $P I=0$ and there is complete risk sharing achieved through private markets. If, on the hand $\operatorname{std}(\log (c))=\operatorname{std}(\log (y)), P I=0$ and private markets do not achieve any risk sharing over and above that achieved by the tax system. We call this situation financial autarky (individuals may still benefit from publicly provided risk sharing).

Now TI can be rewritten as

$$
\begin{aligned}
T I & =1-\frac{s t d(\log (c))}{s t d(\log (e))} \\
& =1-\frac{s t d(\log (y))}{s t d(\log (e))}+\frac{s t d(\log (y))}{s t d(\log (e))}-\frac{s t d(\log (c))}{s t d(\log (e))} \\
& =G I+\frac{s t d(\log (y))-s t d(\log (c))}{s t d(\log (e))} \\
& =G I+(1-G I) * P I
\end{aligned}
$$

Hence total intermediation of risk equals government intermediation of risk plus private intermediation of that part of risk that is not already removed by the tax system. In particular, 
under a proportional tax system $G I=0$ and $T I=P I$. We will report the measures $T I, G I$ and $P I$ for all our policy simulations.

\section{Results for Policy Experiments}

We consider the following policy experiment: Change the tax system from the progressive system found in the calibration section to a proportional system (constant average tax rates), keeping the level of government spending constant. We compare the steady states arising under the two tax systems.

In tables 5-7 we summarize the results from our numerical experiments with the baseline parameterization. The concept of the interest rate for the economy has been discussed in section 5.the concepts of private, government and total intermediation of risk in the previous section. We measure welfare as follows: we first determine which of the tax regimes yields higher ex-ante welfare (where ex-ante welfare is measured as $\left.\int h(w, y) d \Phi=\int w d \Phi\right)$. We then increase the pre-tax endowment of every agent by $x \%$ in the tax regime with lower welfare and report that $x$ (in the column of the preferred tax regime) for which ex-ante welfare in the dominating tax regime coincides with that in the dominated tax regime in which $x \%$ of resources have been added.

For the high value of $\beta=0.972$ the result of our policy experiment is summarized in Table 5 .

Table 5. $\beta=.972$ (High Risk Sharing)

\begin{tabular}{l||l|l}
\multicolumn{1}{c||}{} & \multicolumn{2}{c}{ Tax System } \\
Variable & Progressive & Proportional \\
\hline \hline Interest rate $R-1$ & $2.5 \%$ & $2.7 \%$ \\
Govt. Intermediation GI & 0.211 & 0 \\
Private Intermediation PI & 0.930 & 0.958 \\
Total Intermediation TI & 0.944 & 0.958 \\
Ex Ante Welfare & & $+0.2 \%$
\end{tabular}

A switch from a progressive to a proportional tax system leads to a reduction of publicly enforced risk sharing from 0.211 to 0 (by normalization). But risk sharing achieved via private arrangements increases with the proportional system since the value of autarky 
falls and hence the debt constraints are relaxed. This effect is sufficiently strong to raise total risk sharing with proportional taxes over the level achieved with progressive taxes. Therefore ex-ante welfare increases by $0.2 \%$ with such a tax reform in our simple model. Put differently, the government, in trying to help households to share risk by making the tax system more progressive, achieves exactly the opposite -lower risk sharing and welfare. We want to stress that this result is obtained in a model in which progressive taxes do not distort any margin for the labor-leisure decision. Also note that the interest rate rises with the switch to the proportional system. This is due to the fact that proportional taxes lower the value of default, hence relax the debt constraints. Households can borrow more and the interest rate has to rise to bring borrowing and lending back into equilibrium.

The results from our policy experiment are drastically different for the case of low time discount factors ${ }^{21}$, as can be seen from Tables 6 and 7 .

\begin{tabular}{l||l|l}
\multicolumn{1}{l||}{ Table 6. $\beta=.0212$ (Low Risk Sharing) } \\
Variable & \multicolumn{2}{|c}{ Tax System } \\
\hline \hline Interest rate $R-1$ & $2.5 \%$ & $219 \%$ \\
Govt. Intermediation GI & 0.211 & 0 \\
Private Intermediation PI & 0.0 & 0.008 \\
Total Intermediation $T I$ & 0.211 & 0.008 \\
Ex Ante Welfare & $+36 \%$ &
\end{tabular}

\footnotetext{
${ }^{21}$ One may argue that such low discount factors are a priori unreasonable. Even if we agree it is still instructive to do our policy experiment for an economy where very little private risk sharing is enforceable.
} 


\begin{tabular}{l||l|l}
\multicolumn{1}{l||}{ Table 7. $\beta=.0202$ (Autarky) } \\
Variable & \multicolumn{2}{c}{ Tax System } \\
\hline \hline Interest rate $R-1$ & $2.5 \%$ & $214 \%$ \\
Govt. Intermediation GI & 0.211 & 0 \\
Private Intermediation PI & 0 & 0.008 \\
Total Intermediation TI & 0.211 & 0.008 \\
Ex Ante Welfare & $+37 \%$ &
\end{tabular}

If agents discount the future very heavily, the extent of risk sharing achievable with self-enforceable private contracts is very limited, as the threat of future exclusion from credit markets is not severe for very impatient agents. We see from the tables that the low risk sharing allocation is very similar (in terms of private risk sharing) to the autarkic allocation and it is significantly different from the high risk sharing allocation. The effect of the tax reform on government intermediated risk sharing is similar to the case with high discount factor (by construction). Now, however, private markets are almost completely ineffective in providing risk sharing for both tax systems. Hence total intermediation of risk consists (almost) exclusively of GI. This explains the large welfare losses going from a progressive to a proportional system. Again the interest rate rises (this time very sharply) with the proportional system. Comparing the high risk sharing with the low risk sharing case we conclude that when private markets are very effective in providing risk sharing contracts, then the attempt of the government to do even more may be counterproductive. If, on the other hand, private markets do not work because of it is difficult to enforce private contracts then public risk sharing provided by redistributive taxation leads to potentially large welfare gains. ${ }^{22}$

\section{Risk Sharing as a Function of the Tax System}

So far we have focused on two particular tax systems, a progressive system derived from CEX data and a hypothetical proportional tax system. In this section we show how

\footnotetext{
${ }^{22}$ This suggests that evaluating the level of risk sharing in an economy can be relevant for policy purposes. This, in fact, motivates the analysis in Krueger (1999).
} 
total risk sharing ( and hence aggregate welfare) evolves with (marginal) changes in the progressivity of the tax system in our model. Our parameterization is the same as in section 8 , with $\beta=0.972$. We restrict our discussion to a tax system of the form

$$
\tau\left(e_{j}\right)=a-\frac{b}{e_{j}} \quad j=1, \ldots 5
$$

i.e. to a system with constant marginal tax rate $a$ and a fixed deduction $b$. We estimate $a$ and $b$ by running a regression on our five data points for taxes derived from CEX data. We find $\hat{a}=0.27, \hat{b}=0.11$ The centered $R^{2}$ of this regression equals a surprisingly high 0.996 , so that the progressive tax system used in the last section is almost perfectly approximated by (see figure 6$)^{23}$

$$
\tau\left(e_{j}\right)=0.27-\frac{0.11}{e_{j}} \quad j=1, \ldots 5
$$

i.e. by a constant marginal tax rate of $27 \%$ and a fixed deduction of $11 \%$ of mean income (which we normalized to one in our economy). The implied level of government spending equals $g=0.1622$, which is approximately the same level as the one used in the previous section. We can now vary the degree of progressivity marginally by marginal changes in the fixed deduction $b$, with varying $a$ correspondingly to assure that all tax systems generate the same revenue. ${ }^{24}$ Note that for $b=0$ we are back in our hypothetical proportional tax system from the last section. In figure 8 we plot total risk sharing against the deduction $b$, where total risk sharing is measured as TI. We see that complete risk sharing in this economy can be achieved with two rather extreme policies. One policy that obviously achieves first best is

\footnotetext{
${ }^{23}$ One should note that we only have to fit five data points. Also, remember that our concept of taxes was rather broad, including (the negative of) transfer payments such as welfare and UI benefits.

${ }^{24}$ Note that

$$
\begin{aligned}
g & =\sum_{j=1}^{5} \Pi\left(e_{j}\right) \tau\left(e_{j}\right) \\
& =a-\frac{b}{\sum_{j=1}^{5} \Pi\left(e_{j}\right) e_{j}} \\
& =a-b
\end{aligned}
$$
}

for all tax systems generating the same revenue. We used the fact that $\sum_{j=1}^{5} \Pi\left(e_{j}\right) e_{j}=1$ by normalization of mean income to 1 . 
to tax all income differences away, i.e. making the system extremely progressive and equalize after-tax income of all agents. This is achieved by $b=(1-g)=\bar{y}, a=1$. One the other hand, making the tax system sufficiently regressive makes the punishment from default sufficiently harsh to enforce perfect risk sharing. This occurs for $b<0.08$, i.e. for a poll tax of at least $8 \%$ of mean pre-tax income.

In between these extreme cases the effect on total risk sharing (and hence ex-ante welfare) of a marginal increase in tax progressivity depends on the relative magnitudes of the two effects at work: the direct effect of reducing the variability of after-tax income and the indirect crowding-out effect. For a fixed deduction of $b<0.44$ the crowding-out effect dominates and more progressivity reduces total risk sharing and, therefore, ex-ante welfare. The experiment considered in section 8 falls into this class. For $b>0.44$ the direct effect dominates and more progressive taxes lead to more total risk sharing among individuals and a welfare improvement. Note that the welfare difference between the worst tax regime $(b=0.44)$ and first best amounts to about $1 \%$ of average income.

\section{Exogenous Incomplete Markets}

In this section we want to contrast our finding with the welfare effects of the same reform in a standard exogenous incomplete markets model. In this economy agents are only allowed to trade a single uncontingent bond and they face an exogenously specified constant borrowing limit. There are no enforcement problems of payments in this economy. This type of economies has been widely studied (see Huggett (1993) and Aiyagari (1994) among others) and we consider an economy similar to the one studied by Huggett. The household problem in recursive formulation for this model is (see Huggett (1993) for details):

$$
\begin{aligned}
v(a, y)= & \max _{c \geq 0, a^{\prime} \geq-\underline{b}}(1-\beta) u(c)+\beta \sum_{y^{\prime}} v\left(a^{\prime}, y^{\prime}\right) \pi\left(y^{\prime} \mid y\right) \\
& \text { s.t. } \\
c+a^{\prime}= & y+(1+r) a
\end{aligned}
$$

where $a$ are holdings of the one-period bond and $r$ is the interest rate on these bonds.

To enable comparison with the endogenous incomplete markets economy we calibrate 
this economy to the same observations. In particular, the endowment and tax processes are kept the same. The coefficient of relative risk aversion is again chosen to equal $\sigma=$ 2. We then identify pairs of borrowing limits $\underline{b}$ and time discount factors $\beta$ such that the equilibrium interest rate in this economy equals $2.5 \%$. Table 8 presents the results for our policy experiments for a selection ${ }^{25}$ of $(\underline{b}, \beta)$.

\section{Table 8 Results for Exogenous Incomplete Markets}

\begin{tabular}{|c|c|c|c|c|c|c|c|c|}
\hline \multirow[b]{2}{*}{ Variable } & \multicolumn{2}{|c|}{$\underline{b}=1, \beta=.86$} & \multicolumn{2}{|c|}{$\underline{b}=2, \beta=.9$} & \multicolumn{2}{|c|}{$\underline{b}=5, \beta=.94$} & \multicolumn{2}{|c|}{$\underline{b}=8, \beta=0.95$} \\
\hline & Progr. & Prop. & Progr. & Prop. & Progr. & Prop. & Progr. & Prop. \\
\hline Interest rate & $2.5 \%$ & $-1.5 \%$ & $2.5 \%$ & -0.3 & $2.5 \%$ & $1.5 \%$ & $2.5 \%$ & $1.8 \%$ \\
\hline Govt. Interm. GI & 0.211 & 0 & 0.211 & 0 & 0.211 & 0 & 0.211 & 0 \\
\hline Private Interm. $P I$ & 0.161 & 0.250 & 0.237 & 0.335 & 0.330 & 0.429 & 0.344 & 0.442 \\
\hline Total Interm.TI & 0.338 & 0.250 & 0.398 & 0.335 & $0 . .471$ & 0.429 & 0.483 & 0.442 \\
\hline Ex Ante Welfare & $+10.9 \%$ & & $+7.9 \%$ & & $+5.0 \%$ & & $+4.5 \%$ & \\
\hline
\end{tabular}

In this economy a switch from a progressive to a proportional tax system induces (large) welfare losses. Redistributive taxes act as a partial substitute for private insurance markets that are exogenously assumed to be missing. Removing this partial substitute for private markets leads to negative welfare consequences. This is true regardless of how high the borrowing limit is specified. In contrast to the endogenous incomplete markets model a tax reform does not (by assumption) change the assets that can be traded nor the extent to which they can be traded. Therefore the crowding-out effect that was crucial in the previous section cannot occur in this economy.

Another important difference between this economy and the one analyzed previously is the impact that the change in tax system has on the interest rate. In the debt-constrained economy a shift from progressive to proportional taxes causes an increase in the interest rate while in the economy with exogenous incomplete markets interest falls in response to

\footnotetext{
${ }^{25}$ There is a limit as to how negative one can chose $\underline{b}$. This limit is given by the constraint that agents with $y=y_{\min }$ and $a=-\underline{b}$ can attain nonnegative consumption by setting $a^{\prime}=-\underline{b}$ at the equilibrium interest rate (for both tax systems). This limit on $\underline{b}$ turns out be approximately equal to 8 .
} 
the change in the tax system. The intuition for this result is as follows: the change in taxes increases the volatility of the income process. This, by making autarky less attractive, relaxes the borrowing constraints faced by the agents in the debt-constraint economy. Since agents borrow more, the interest rate has to rise to clear the credit markets. In the exogenous incomplete markets, on the other hand, agents, facing higher volatility of output, increase their precautionary saving. Since their borrowing limit is unaffected now a decline in the equilibrium interest rate is needed to clear the credit market.

\section{Conclusions}

We have presented a model that highlights a new channel through which different taxation schemes can affect private financial markets. Although in our model taxes do not directly affect labor-leisure and wealth accumulation decisions they affect the functioning of private financial markets by changing the incentives to default on private contracts. We have shown that when private insurance markets are active, risk sharing provided through taxes crowds out private risk sharing. In order to gain some insights into the magnitude of this effect we calibrate our very simple model to US income and tax data so that we can quantify the effects of changes in the progressivity of taxation. Our experiments indicate that the magnitude of the crowding out can vary a great deal. In particular, we find that this magnitude depends on the level of risk sharing that is achieved via private contracts. When risk sharing provided by private markets is low, crowding out is small and redistribution through taxes is welfare improving, while in high risk sharing regimes redistribution through taxes crowds out private financial markets more than one to one, and therefore is welfare reducing.

In contrast, if private insurance markets are assumed to be missing for reasons exogenous from the model, as in a standard exogenous incomplete markets model, a tax reform that reduces the variance of after-tax income serves as a partial substitute for private insurance markets and leads to unambiguous welfare gains. This, to us, demonstrates that when analyzing a tax policy reform it is important to take into account the interaction between the tax policy and the functioning of private insurance markets.

Finally, we would like to stress that our analysis focuses only on a particular, so far 
largely ignored, channel through which the tax system affects welfare. By trying to isolate this channel we necessarily have to abstract from many features that a more complete discussion of potential tax reforms should incorporate. 


\section{References}

[1] Aiyagari, R. (1994): "Uninsured Idiosyncratic Risk and Aggregate Saving," Quarterly Journal of Economics, 109, 659-684.

[2] Alvarez, F. and U. Jermann (1998a): "Asset Pricing when Risk Sharing is Limited by Default," mimeo.

[3] Alvarez, F. and U. Jermann (1998b): "Quantitative Implications for Asset Pricing when Risk Sharing is Limited by Default," mimeo.

[4] Atkeson, A. and R. Lucas (1992): "On Efficient Distribution with Private Information," Review of Economic Studies, 59, 427-453

[5] Atkeson, A. and R. Lucas (1995): "Efficiency and Equality in a Simple Model of Efficient Unemployment Insurance," Journal of Economic Theory, 66, 64-88.

[6] Attanasio, O. and S. Davis (1996): "Relative Wage Movements and the Distribution of Consumption," Journal of Political Economy, 104, 1227-1262.

[7] Attanasio, O. and V. Rios-Rull (1999): "Consumption Smoothing and Extended Families: The Role of Government Sponsored Insurance," mimeo, University of Pennsylvania.

[8] Barsky, R., G. Mankiw and S. Zeldes (1986): "Ricardian Consumers with Keynesian Properties," American Economic Review, 76, 676-691.

[9] Bewley, T. (1986): "Stationary Monetary Equilibrium with a Continuum of Independently Fluctuating Consumers," in Contributions to Mathematical Economics in Honor of Gerard Debreu, edited by W. Hildenbrand and A. Mas-Colell, Amsterdam: North Holland.

[10] Chan, L. (1983): "Uncertainty and the Neutrality of Government Financing Policy," Journal of Monetary Economics, 11, 351-372.

[11] Cole, H. and N. Kocherlakota (1998): "Efficient Allocations With Hidden Income and Hidden Storage," Federal Reserve Bank of Minneapolis, Staff Report 238. 
[12] Cochrane, J. (1991): "A Simple Test of Consumption Insurance," Journal of Political Economy, 97,957-976

[13] Coleman, J. (1990): "Solving the Stochastic Growth Model by Policy Function Iteration," Journal of Business and Economic Statistics, 8, 27-29.

[14] Constantinides, G. and D. Duffie (1996): "Asset Pricing with Heterogeneous Consumers," Journal of Political Economy, 104, 219-240.

[15] Deaton, A. and C. Paxon (1994): "Intertemporal Choice and Inequality," Journal of Political Economy, 102, 437-467.

[16] Díaz-Giménez, J., V.Quadrini and J.V. Ríos-Rull (1997): "Dimensions of Inequality: Facts on the U.S. Distributions of Earnings, Income, and Wealth", Federal Reserve Bank of Minneapolis Quarterly Review, 21, 3-21

[17] Green E. (1987), "Lending and the Smoothing of Uninsurable Income," in E. Prescott and N. Wallace (eds.), Contractual Arrangements for Intertemporal Trade, University of Minnesota Press, Minneapolis.

[18] Hayashi, F. , J. Altonji, and L. Kotlikoff (1996): "Risk Sharing Between and Within Families," Econometrica, 64, 261-294.

[19] Huggett, M. (1993): "The Risk-Free Rate in Heterogeneous-Agent Incomplete-Insurance Economies," Journal of economic Dynamics and Control, 17, 953-969.

[20] Kehoe, T. and D. Levine (1993): "Debt Constrained Asset Markets," Review of Economic Studies, 60, 865-888.

[21] Kehoe, T. and D. Levine (1998): "Incomplete Markets versus Debt Constrained Markets," mimeo.

[22] Kimball, M. and G. Mankiw (1989): "Precautionary Saving and the Timing of Taxes," Journal of Political Economy, 97, 863-879.

[23] Kocherlakota, N. (1996): "Implications of Efficient Risk Sharing without Commitment," Review of Economic Studies, 63, 595-609. 
[24] Krueger, D. (1998): "Risk Sharing with Incomplete Markets," mimeo.

[25] Mace, B. (1991): "Full Insurance in the Presence of Aggregate Uncertainty," Journal of Political Economy, 99, 928-956.

[26] Mehra, R. and E. Prescott (1985): "The Equity Premium: A Puzzle," Journal of Monetary Economics, 15, 145-161.

[27] Lusardi, A. (1996): "Permanent Income, Current Income and Consumption: Evidence From Two Panel Data Sets," Journal of Business and Economics Statistics, 14, 81-90

[28] Nelson, J. (1994): "On Testing for Full Insurance Using Consumer Expenditure Survey Data," Journal of Political Economy, 102, 384-394.

[29] Stokey, N. and R. Lucas, with E. Prescott (1989): "Recursive Methods in Economic Dynamics," Harvard University Press, Cambridge.

[30] Storesletten, K., C. Telmer and A. Yaron (1998): "Persistent Idiosyncratic Shocks and Incomplete Markets," mimeo.

[31] Varian, H. (1980): "Redistributive Taxation as Social Insurance," Journal of Public Economics, 14, 49-68. 


\section{Appendix}

\section{A1. Figures}
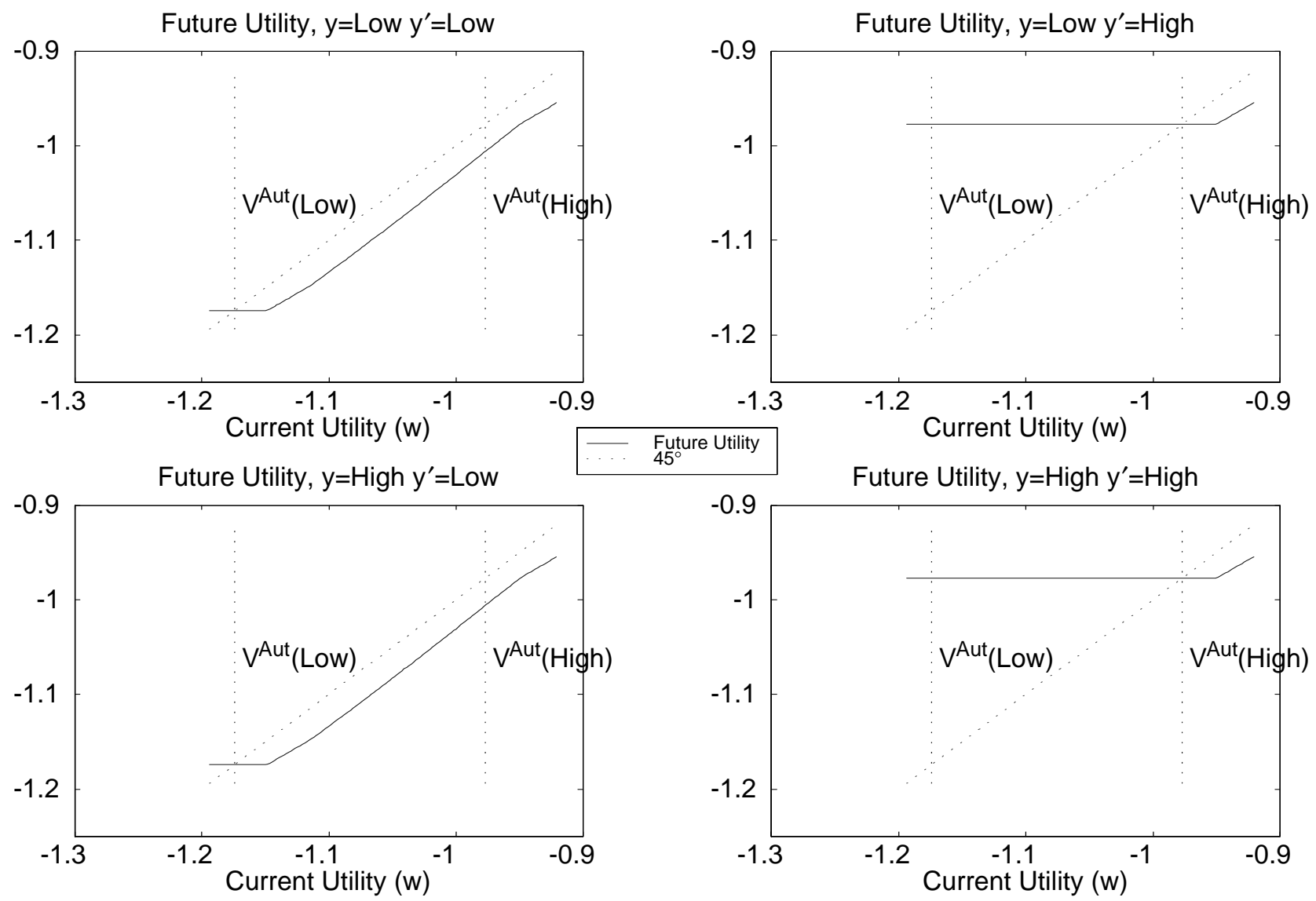

Figure 1: 


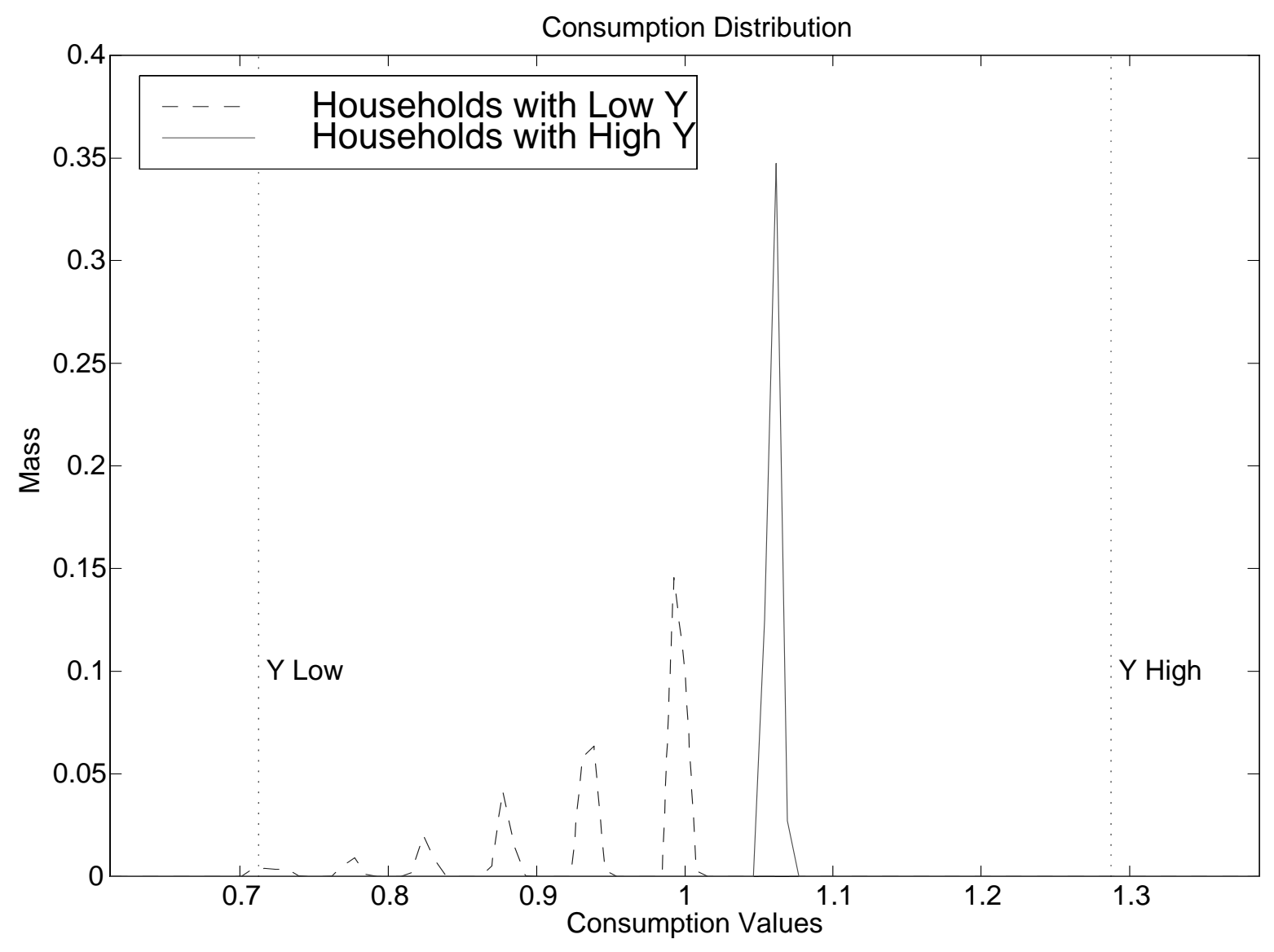

Figure 2: 


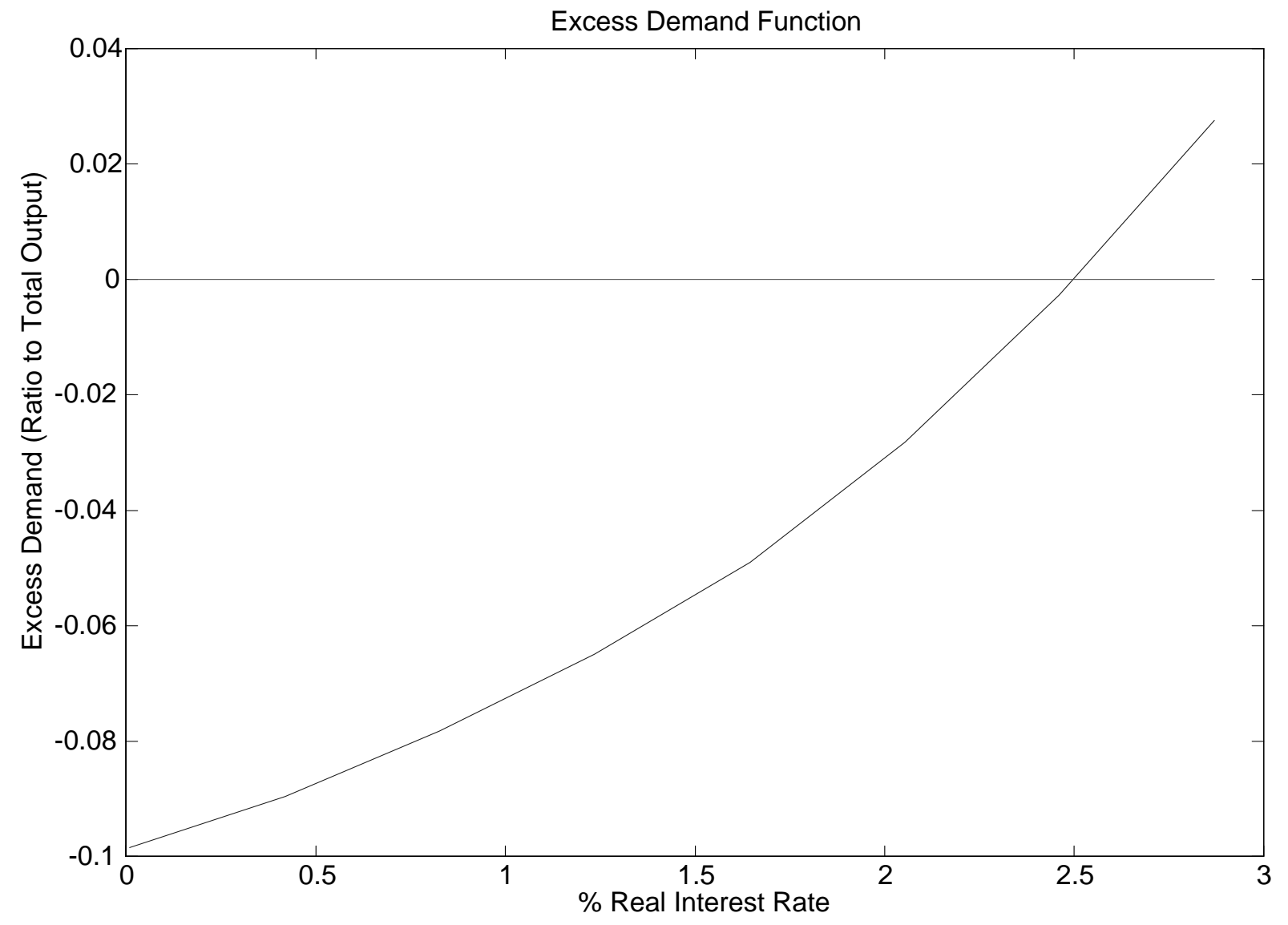

Figure 3: 


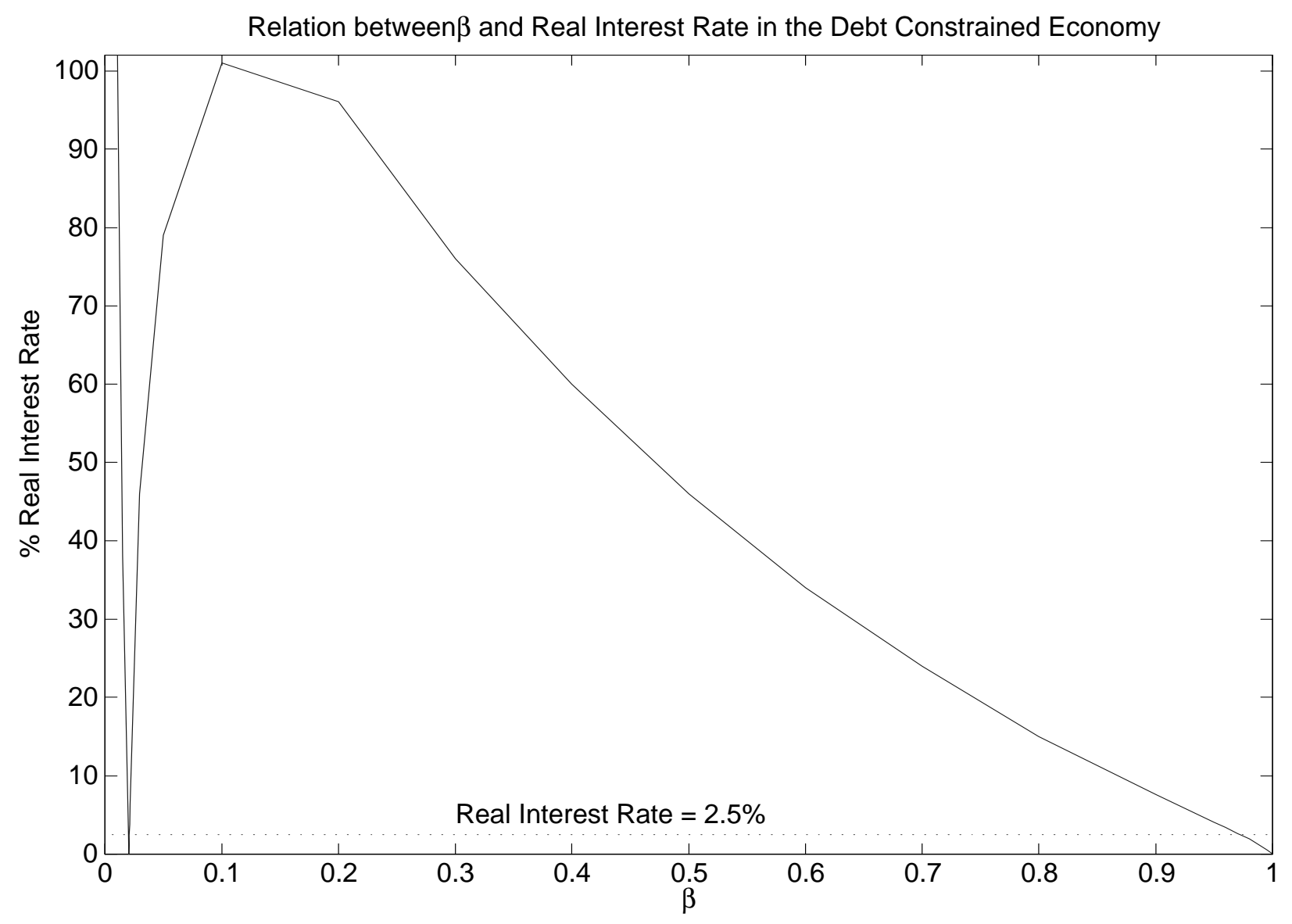

Figure 4: 


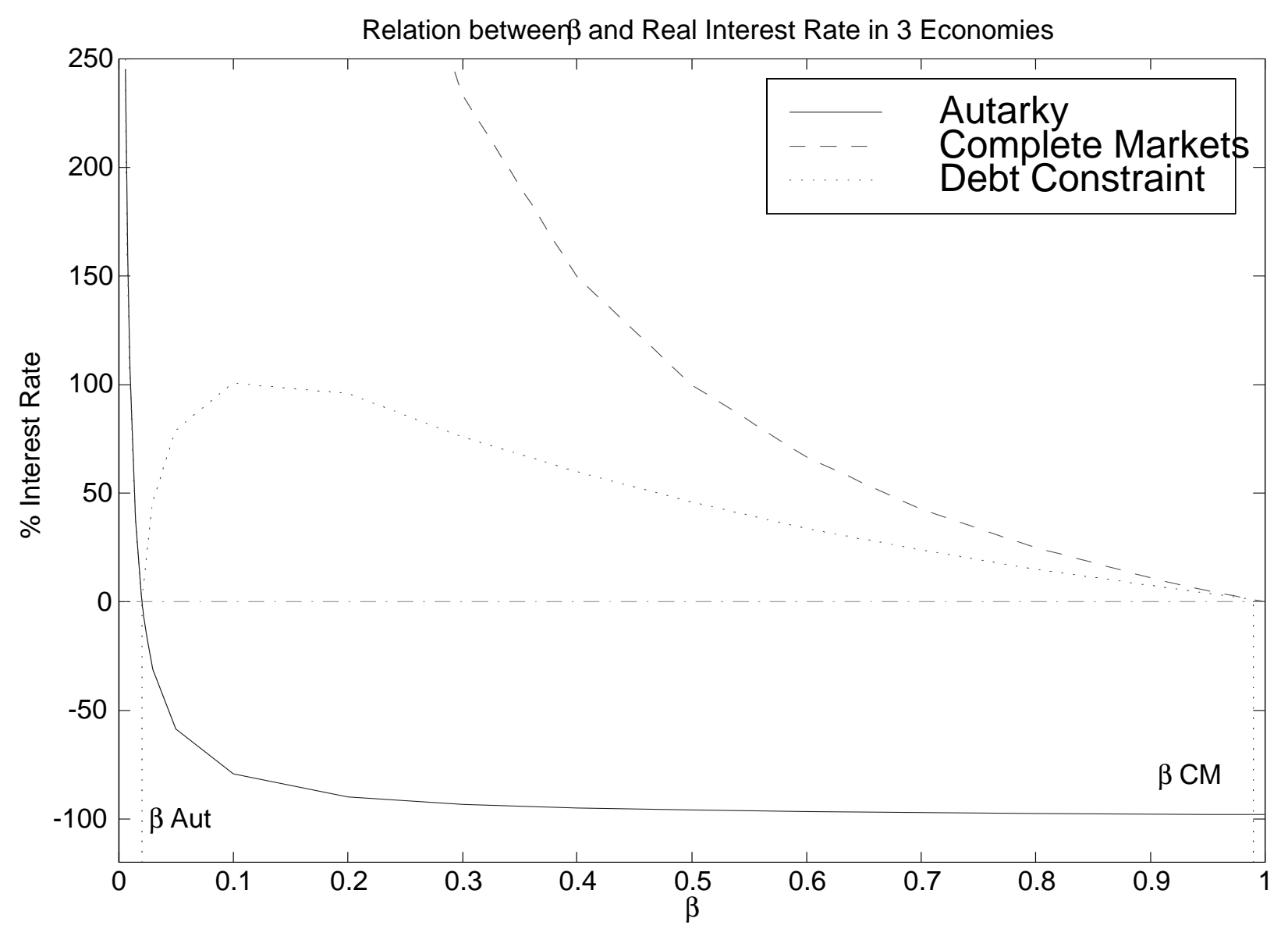

Figure 5: 


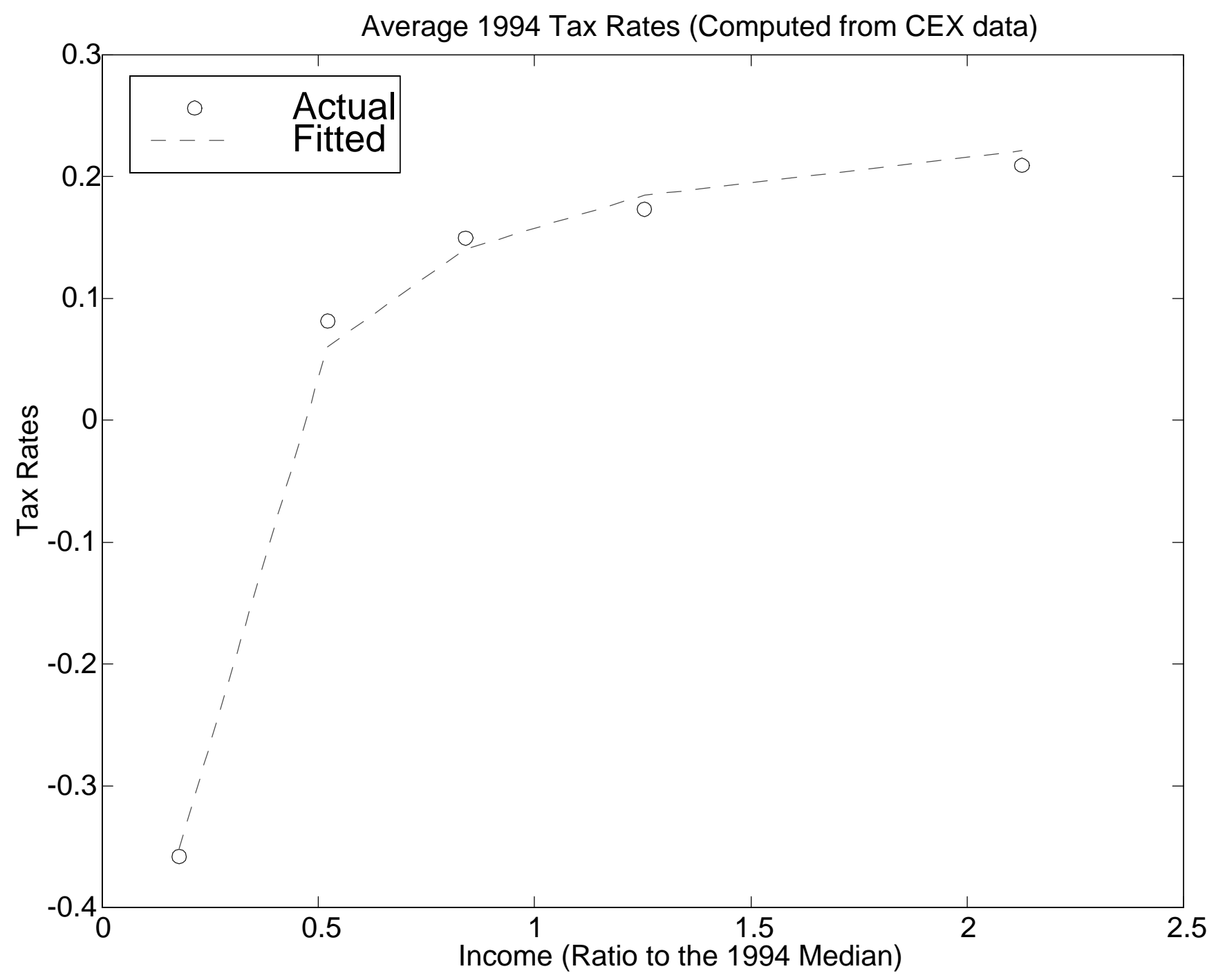

Figure 6: 


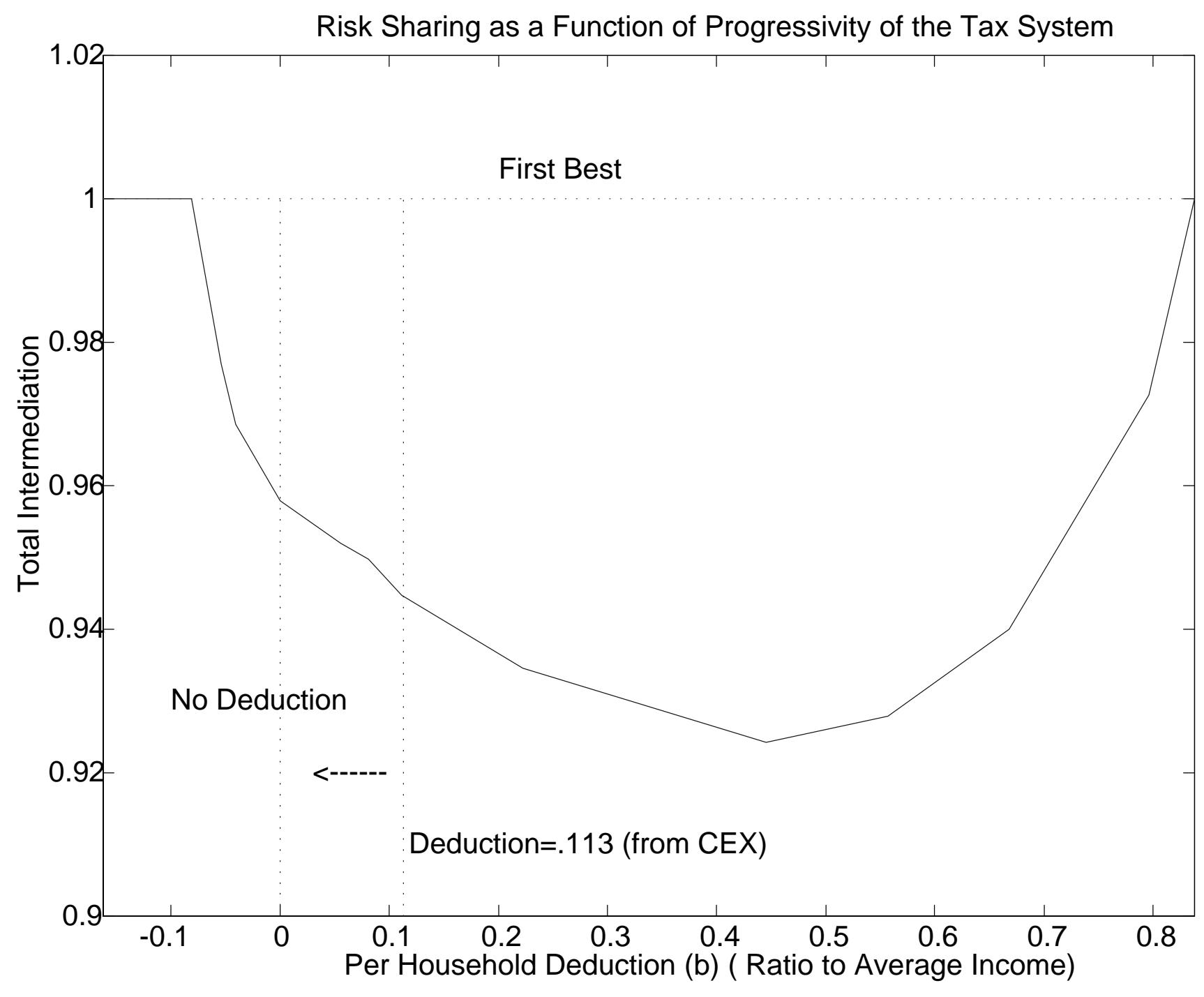

Figure 7: 


\section{A2. Proofs}

\section{Proof of Theorem 1.:}

To show efficiency we first need to show that $\left\{h_{t}\left(w_{0}, y^{t}\right)\right\}$ is constrained feasible with respect to $\Phi_{0}$. By assumption the allocation satisfies feasibility (equation 6) and since it solves $C P P$ also satisfies 5, 6, and 7. It is therefore constrained feasible. Now we need to show that there does not exist another allocation $\left\{\hat{h}_{t}\left(w_{0}, y^{t}\right)\right\}_{t=0}^{\infty}$ that is constrained feasible with respect to $\Phi_{0}$ and such that

$$
\sum_{y^{t}} \int C\left(\hat{h}_{t}\left(w_{0}, y^{t}\right)\right) \pi\left(y^{t} \mid y_{0}\right) d \Phi_{0}<\sum_{y^{t}} \int C\left(\hat{h}_{t}\left(w_{0}, y^{t}\right)\right) \pi\left(y^{t} \mid y_{0}\right) d \Phi_{0} \text { for some } t
$$

Suppose this is the case. Since $\left\{h_{t}\left(w_{0}, y^{t}\right)\right\}$ solves $C P P$ for all $w_{0}, y_{0}$ we have

$$
\begin{aligned}
& \left(1-\frac{1}{R_{0}}\right) C\left(h_{0}\left(w_{0}, y_{0}\right)\right)+\sum_{t=1}^{\infty}\left(1-\frac{1}{R_{t}}\right) \prod_{s=0}^{t-1}\left(\frac{1}{R_{s}}\right) \sum_{y^{t} \mid y_{0}} C\left(h_{t}\left(w_{0}, y^{t}\right)\right) \pi\left(y^{t} \mid y_{0}\right) \\
\leq & \left(1-\frac{1}{R_{0}}\right) C\left(\hat{h}_{0}\left(w_{0}, y_{0}\right)\right)+\sum_{t=1}^{\infty}\left(1-\frac{1}{R_{t}}\right) \prod_{s=0}^{t-1}\left(\frac{1}{R_{s}}\right) \sum_{y^{t} \mid y_{0}} C\left(\hat{h}_{t}\left(w_{0}, y^{t}\right)\right) \pi\left(y^{t} \mid y_{0}\right)
\end{aligned}
$$

where the left hand side of equation A2 is finite ${ }^{26}$. Integrating both sides of A2 with respect to $\Phi_{0}$ One has

$$
\begin{aligned}
& \text { (A3) } \int\left\{\left(1-\frac{1}{R_{0}}\right) C\left(h_{0}\left(w_{0}, y_{0}\right)\right)+\sum_{t=1}^{\infty}\left(1-\frac{1}{R_{t}}\right) \prod_{s=0}^{t-1}\left(\frac{1}{R_{s}}\right) \sum_{y^{t} \mid y_{0}} C\left(h_{t}\left(w_{0}, y^{t}\right)\right) \pi\left(y^{t} \mid y_{0}\right)\right\} d \Phi_{0} \\
& \leq \int\left\{\left(1-\frac{1}{R_{0}}\right) C\left(\hat{h}_{0}\left(w_{0}, y_{0}\right)\right)+\sum_{t=1}^{\infty}\left(1-\frac{1}{R_{t}}\right) \prod_{s=0}^{t-1}\left(\frac{1}{R_{s}}\right) \sum_{y^{t} \mid y_{0}} C\left(\hat{h}_{t}\left(w_{0}, y^{t}\right)\right) \pi\left(y^{t} \mid y_{0}\right)\right\} d \Phi_{0}
\end{aligned}
$$

From the fact that for $\left\{h_{t}\left(w_{0}, y^{t}\right)\right\}$ feasibility holds with equality for all $t$, that $\left\{\hat{h}_{t}\left(w_{0}, y^{t}\right)\right\}$

\footnotetext{
${ }^{26}$ This is guaranteed since we can always pick a constant $h_{t}\left(w_{0}, y^{t}\right)=\max \left(w_{0}, \max _{y} V^{A u t}(y)\right)$. Such a policy satisfies all the constraints of $C P P$ and since $\max \left(w_{0}, \max _{y} V^{A u t}(y)\right) \in D$ and condition 3 . in Theorem 1 is satisfied the value of the minimization problem is finite.
} 
is constrained feasible and from A1 one obtains:

$$
\sum_{y^{t} \mid y_{0}} \int C\left(\hat{h}_{t}\left(w_{0}, y^{t}\right)\right) \pi\left(y^{t} \mid y_{0}\right) d \Phi_{0} \leq \sum_{y^{t} \mid y_{0}} \int y_{t} \pi\left(y^{t} \mid y_{0}\right) d \Phi_{0}=\sum_{y^{t} \mid y_{0}} \int C\left(h_{t}\left(w_{0}, y^{t}\right)\right) \pi\left(y^{t} \mid y_{0}\right) d \Phi_{0}
$$

for all $t$ with the inequality being strict for some $t$. Multiplying each inequality by the appropriate term $\left(1-\frac{1}{R_{t}}\right) \prod_{s=0}^{t-1}\left(\frac{1}{R_{s}}\right)>0$ and summing over all $t$ we obtain A3, but with the inequality reversed and strict, a contradiction

\section{Proof of Lemma 1.:}

For every $(w, y) \in A$ the objective function in 19 is continuous in $h, g_{y^{\prime}}$ and the constraint set is compact and non-empty; therefore the minimum exists. $V$ is bounded and since $\underline{h}(w, y) \leq h \leq \bar{h}(w, y), C(h)$ is bounded as well. It follows that $T_{R} V$ is a bounded function. The fact that $T_{R} V$ is continuous follows from the Theorem of the maximum (note that the constraint set is continuous in $w$ ). It is also easy to show that since $R>1$ the operator $T_{R}$ satisfies the hypotheses of Blackwell's theorem and thus is a contraction with modulus $\frac{1}{R}$

\section{Proof of Lemma 2.:}

For the first part we note that $C(A)$ (together with the sup-norm) is a complete metric space and that the set of bounded continuous nondecreasing (in its first argument) functions on $A, C^{\prime}\left(A_{i}\right)$, is a closed subset of $C(A)$ and that the set of bounded continuous strictly increasing functions, $C^{\prime \prime}(A)$, satisfies $C^{\prime \prime}(A) \subset C^{\prime}(A)$. By Lemma 1. $T_{R}$ is a contraction mapping. Hence by Corollary 1 of Stokey et al., p. 52, it is sufficient to show that, whenever $V_{R} \in C^{\prime}(A)$, then $T_{R} V_{R} \in C^{\prime \prime}(A)$. Fix $w, \hat{w}$ with $\underline{w} \leq w<\hat{w} \leq \bar{w}$. We need to show that $\left(T_{R} V_{R}\right)(w)<\left(T_{R} V_{R}\right)(\hat{w})$. Let $\hat{h}, \hat{g}_{y^{\prime}}$ be the optimal choices for $\hat{w}$. The choices $g_{y^{\prime}}=\hat{g}_{y^{\prime}}$ and $h=\hat{h}-\hat{w}+w<\hat{h}$ are feasible for $w$ and therefore

$$
\begin{aligned}
\left(T_{R} V_{R}\right)(\hat{w}) & =\left(\frac{R-1}{R}\right) C(\hat{h})+\frac{1}{R} \sum_{y^{\prime} \in Y} \pi\left(y^{\prime}\right) V_{R}\left(\hat{g}_{y^{\prime}}\right) \\
& >\left(\frac{R-1}{R}\right) C(h)+\frac{1}{R} \sum_{y^{\prime} \in Y} \pi\left(y^{\prime}\right) V_{R}\left(g_{y^{\prime}}\right) \\
& \geq\left(T_{R} V_{R}\right)(w)
\end{aligned}
$$


To prove that $V_{R}$ is convex we note that the set of bounded continuous convex functions, $C^{\prime \prime \prime}(A)$ is a closed subset of $C(A)$. Again by Corollary 1 of Stokey et al., p. 52 , it is sufficient to show that if $V_{R} \in C^{\prime \prime \prime}(A)$, then $\left(T_{R} V_{R}\right)$ is convex in its first argument. So we have to show that for all $w, \hat{w} \in A$ with $w \neq \hat{w}$, and all $\lambda \in(0,1)$, $\left(T_{R} V\right)(\lambda w+(1-\lambda) \hat{w}) \leq \lambda\left(T_{R} V\right)(w)+(1-\lambda)\left(T_{R} V\right)(\hat{w})$. Let $\hat{h}, \hat{g}_{y}$ be the optimal choices for $\hat{w}$ and $h, g_{y}$ be the optimal choices for $w$ and define $h^{\lambda}=\lambda h+(1-\lambda) \hat{h}, g_{y}^{\lambda}=\lambda g_{y}+(1-\lambda) \hat{g}_{y}$. Since $h^{\lambda}, g_{y}^{\lambda}$ are feasible for $(\lambda w+(1-\lambda) \hat{w}, y)$, and

$$
\begin{aligned}
& \left(T_{R} V_{R}\right)(\lambda w+(1-\lambda) \hat{w}) \\
\leq & \left(\frac{R-1}{R}\right) C\left(h^{\lambda}\right)+\frac{1}{R} \sum_{y^{\prime} \in Y} \pi\left(y^{\prime}\right) V\left(g_{y^{\prime}}^{\lambda}\right) \\
\leq & \left(\frac{R-1}{R}\right)(\lambda C(h)+(1-\lambda) C(\hat{h}))+\frac{1}{R} \sum_{y^{\prime} \in Y} \pi\left(y^{\prime}\right)\left(\lambda V\left(g_{y^{\prime}}\right)+(1-\lambda) V\left(\hat{g}_{y^{\prime}}\right)\right) \\
= & \lambda\left(T_{R} V_{R}\right)(w)+(1-\lambda)\left(T_{R} V_{R}\right)(\hat{w})
\end{aligned}
$$

by convexity of $V$ in its first argument and strict convexity of $C$.

We want to show that the fixed point of $T_{R}, V$, is strictly convex on $A$. We know that $V$ is convex, continuous and strictly increasing. These facts imply that $V$ is differentiable almost everywhere on $A$ and that for the countable number of points at which $V$ is not differentiable, right hand derivatives $V_{+}^{\prime}$ and left hand derivatives $V_{-}^{\prime}$ exist (although need not coincide).

Now suppose that $V$ is not strictly convex on $A$. Then there exists an interval $I \subseteq A$ such that $V$ is linear on $I$. Take $w, w^{\prime} \in I$ with $w<w^{\prime}$. From the envelope theorem for any solution $\left\{g_{y^{\prime}}(w)\right\},\left\{g_{y^{\prime}}\left(w^{\prime}\right)\right\}$

$$
\begin{aligned}
a & =V^{\prime}(w)=V^{\prime}\left(w^{\prime}\right) \\
& =\frac{R-1}{R(1-\beta)} C^{\prime}\left(\frac{w-\beta \sum_{y^{\prime}} \pi\left(y^{\prime}\right) g_{y^{\prime}}(w)}{1-\beta}\right) \\
& =\frac{R-1}{R(1-\beta)} C^{\prime}\left(\frac{w^{\prime}-\beta \sum_{y^{\prime}} \pi\left(y^{\prime}\right) g_{y^{\prime}}\left(w^{\prime}\right)}{1-\beta}\right)
\end{aligned}
$$

for some $a>0$. Hence there exists $\bar{y}$ such that $U^{A u t}(\bar{y}) \leq g_{\bar{y}}(w)<g_{\bar{y}}\left(w^{\prime}\right)$. From the first order 
conditions, combining with the envelope condition

$$
\begin{aligned}
a \beta R & \leq V_{+}^{\prime}\left(g_{\bar{y}}(w)\right) \\
V_{-}^{\prime}\left(g_{\bar{y}}\left(w^{\prime}\right)\right) & \leq a \beta R \leq V_{+}^{\prime}\left(g_{\bar{y}}\left(w^{\prime}\right)\right)
\end{aligned}
$$

By convexity of $V$ and the fact that $g_{\bar{y}}(w)<g_{\bar{y}}\left(w^{\prime}\right)$ it follows that

$$
a \beta R \leq V_{+}^{\prime}\left(g_{\bar{y}}(w)\right) \leq V_{-}^{\prime}\left(g_{\bar{y}}\left(w^{\prime}\right)\right) \leq a \beta R
$$

Hence $V$ is linear on $I^{\prime}=\left(g_{\bar{y}}(w), g_{\bar{y}}\left(w^{\prime}\right)\right) \subseteq A$ with slope $a g<a$. Repeating the above argument one shows that there exists interval $I^{(n)}$ such that $V$ is linear on $I^{(n)}$ with slope $a g^{n}$, for all $n>1$.

Now let $d=\frac{R-1}{R(1-\beta)} C^{\prime}(\underline{h}(\underline{w}))>0$ and pick $n$ such that $a g^{n}<d$. Then for all $w \in I^{(n)}$, using the envelope condition

$$
a g^{n}=V^{\prime}(w)=\frac{R-1}{R(1-\beta)} C^{\prime}(h(w))<d=\frac{R-1}{R(1-\beta)} C^{\prime}(\underline{h}(\underline{w}))
$$

Therefore $h(w)<\underline{h}(\underline{w})$, which is impossible. Hence $V$ cannot contain a linear segment on A.

\section{Proof of Lemma 3.:}

The fact that $T_{R} V$ is strictly increasing and strictly convex follows from the properties of $V$. The choice variables $h$ and $g_{y^{\prime}}$ are constrained to lie in compact and convex intervals, and by assumption the objective function is strictly convex. Hence the minimizers are unique. Since the constraint set is continuous in $w$, the theorem of the maximum applies and $T_{R} V$ is continuous and $h(w), g_{y^{\prime}}(w)$ are upper hemicontinuous correspondences. Since $h(w), g_{y^{\prime}}(w)$ are functions, they are continuous 


\section{Proof of Lemma 4.:}

Consider the following sequence of functions $\left\{V^{n}\right\}_{n=0}^{\infty}$, defined recursively as:

$$
\begin{aligned}
V^{0}(w) & =C(w) & \forall w \in A \\
V^{n+1}(w) & =\left(T^{R} V^{n}\right)(w) & \forall w \in A
\end{aligned}
$$

From corollary 1. we know that this sequence converges uniformly to the unique fixed point $V_{R}$ of $T_{R}$. Also Lemma 3. assures that each $V^{n}$ is continuous, strictly increasing and strictly convex (as by assumption $C$ possesses these properties) and that the associated policies $h^{n}(w)$ and $g_{y^{\prime}}^{n}(w)$ are continuous functions. From 24 we have (as $C$ is continuously differentiable by assumption) that each $V^{n}$ is differentiable and that this derivative is continuous, since $h^{n-1}(w)$ is a continuous function. Now we will establish that $V_{R}$ is continuously differentiable.

From Lemmas 3., 2. and corollary 1. we know that each $V^{n}$ as well as $V_{R}$ are strictly convex and continuous and that the sequence $\left\{V^{n}\right\}_{n=0}^{\infty}$ converges to $V_{R}$ uniformly. Also $A$ is compact. Then by theorem 3.8 of Stokey et al., p. 64, the sequences $\left\{h^{n}(w), g_{y^{\prime}}^{n}(w)\right\}_{n=1}^{\infty}$ converge uniformly to the optimal policies associated with $V_{R}, h^{R}(w)$ and $g_{y^{\prime}}^{R}(w)$, respectively. Therefore from $24\left(T_{R} V^{n}\right)^{\prime}$ converges to $\frac{(R-1)}{R(1-\beta)} C^{\prime}\left(h^{R}(w)\right)$ uniformly. Since $\left\{V^{n}\right\}_{n=0}^{\infty}$ converges to $V_{R}$ uniformly, we have that $V_{R}$ is differentiable, with

$$
\left(V_{R}\right)^{\prime}(w)=\frac{(R-1)}{R(1-\beta)} C^{\prime}\left(h^{R}(w)\right)
$$

\section{Proof of Lemma 5.:}

We want to show that for all $\underline{w} \leq w<\hat{w} \leq \bar{w}, h(w)<h(\hat{w})$. Suppose, to the contrary, $h(w) \geq h(\hat{w})$. Then from 23

$$
V^{\prime}\left(g_{y^{\prime}}(w)\right) \geq V^{\prime}\left(g_{y^{\prime}}(\hat{w})\right)
$$

for all $y^{\prime}$ such that $g_{y^{\prime}}(\hat{w})>U^{A u t}\left(y^{\prime}\right)$, and hence $U^{A u t}\left(y^{\prime}\right)<g_{y^{\prime}}(\hat{w}) \leq g_{y^{\prime}}(w)$ for all those $y^{\prime}$ by 
strict convexity of $V$. But then from the promise keeping constraint there must exist $\bar{y}^{\prime}$ such that $g_{\bar{y}^{\prime}}(w)<g_{\bar{y}^{\prime}}(\hat{w})=U^{A u t}\left(\bar{y}^{\prime}\right)$, a violation of the debt constraint. We obtain a contradiction to the assumption that $h(w, y)>h(\hat{w}, y)$

\section{Proof of Lemma 6.:}

Again let $\underline{w} \leq w<\hat{w} \leq \bar{w}$. Under the assumptions made $h$ is strictly increasing in $w$. Therefore $C^{\prime}(h(w))<C^{\prime}(h(\hat{w}))$. First suppose that $g_{y^{\prime}}(w)>U^{A u t}\left(y^{\prime}\right)$. Then from 23 we have

$$
V^{\prime}\left(g_{y^{\prime}}(w)\right)<V^{\prime}\left(g_{y^{\prime}}(\hat{w})\right)
$$

and from the strict convexity of $V$ it follows that $g_{y^{\prime}}(\hat{w})>g_{y^{\prime}}(w)$. Obviously, if $g_{y^{\prime}}(w)=$ $U^{A u t}\left(y^{\prime}\right)$ then $g_{y^{\prime}}(\hat{w}) \geq g_{y^{\prime}}(w)$, i.e. either $g_{y^{\prime}}(\hat{w})>g_{y^{\prime}}(w)$ or $g_{y^{\prime}}(w)=g_{y^{\prime}}(\hat{w})=U^{A u t}\left(y^{\prime}\right)$

\section{Proof of Lemma 8.:}

From Lemmas 2. and 4. $V_{R}$ is strictly convex and differentiable. By assumption $g_{y^{\prime}}(w)>$ $U^{\text {Aut }}\left(y^{\prime}\right)$. Then combining 23 and 24 we obtain

$$
\beta R\left(V_{R}\right)^{\prime}(w)=\left(V_{R}\right)^{\prime}\left(g_{y^{\prime}}(w)\right)
$$

Since $R<\frac{1}{\beta}$ we have $V^{\prime}(w)>V^{\prime}\left(g_{y^{\prime}}(w)\right)$, and by strict convexity of $V_{R}$ the first result follows. Hence $g_{y^{\prime}}($.$) are always strictly below the 45^{0}$ line in their strictly increasing part. On the other hand $g_{y^{\prime}}(w) \geq U^{A u t}\left(y^{\prime}\right)$ for all $w$. Hence for $w<U^{A u t}\left(y^{\prime}\right), g_{y^{\prime}}(w)=U^{A u t}\left(y^{\prime}\right)>w$. By continuity of $g_{y^{\prime}}(),. g_{y^{\prime}}\left(U^{A u t}\left(y^{\prime}\right)\right)=U^{A u t}\left(y^{\prime}\right)$ and from the first result $g_{y^{\prime}}(w)<w$ for all $w>U^{A u t}\left(y^{\prime}\right)$

\section{Proof of Theorem 2.:}

Take $\bar{w}=\max _{y} U^{A u t}(y)+\varepsilon$, for $\varepsilon>0$ arbitrarily small. If $g_{y^{\prime}}(w)>U^{A u t}\left(y^{\prime}\right)$, then the previous Lemma yields the result. If $g_{y^{\prime}}(w)=U^{A u t}\left(y^{\prime}\right)$, then $g_{y^{\prime}}(w)=U^{A u t}\left(y^{\prime}\right) \leq$ $\max _{y} U^{A u t}(y)<\bar{w}$ 


\section{Proof of Theorem 3.:}

For any allocation $\sigma=\left\{h_{t}\left(w_{0}, y^{t}\right)\right\}_{t=0}^{\infty}$ define

$$
\begin{aligned}
U_{t}\left(w_{0}, y^{t}, \sigma\right) & =(1-\beta)\left(h_{t}\left(w_{0}, y^{t}\right)+\sum_{s>t}^{\infty} \sum_{y^{s}} \beta^{s-t} \pi\left(y^{s}\right) h_{t}\left(w_{0}, y^{s}\right)\right) \\
U_{t}^{A u t}\left(y_{t}\right) & =(1-\beta)\left(u\left(y_{t}\right)+\sum_{s>t}^{\infty} \sum_{y^{s}} \beta^{s-t} \pi\left(y^{s}\right) u\left(y_{s}\right)\right)
\end{aligned}
$$

By theorem 4.3 in Stokey et al. (the assumption of which are satisfied as $C(w) \geq 0$, all $w$ ), for all $w_{0} \in W$ and all $y_{0} \in Y$, the solution to the functional equation, $V_{R}$ satisfies

$$
V_{R}\left(w_{0}\right)=\inf _{\left\{h_{t}\left(w_{0}, y^{t}\right), w_{t}\left(w_{0}, y^{t}\right)\right\}}\left(1-\frac{1}{R}\right) C\left(h_{0}\left(w_{0}, y_{0}\right)\right)+\sum_{t=1}^{\infty}\left(1-\frac{1}{R}\right) \frac{1}{R^{t}} \sum_{y^{t}} C\left(h_{t}\left(w_{0}, y^{t}\right)\right) \pi\left(y^{t}\right)
$$

(A10)

$$
\text { s.t } w_{t}\left(w_{0}, y^{t}\right)=(1-\beta) h_{t}\left(w_{0}, y^{t}\right)+\beta \sum_{y^{t+1}} \pi\left(y_{t+1}\right) w_{t+1}\left(w_{0}, y^{t+1}\right) \quad \text { all } t
$$

$(\mathrm{A} 11 \mathrm{a}) U_{t}^{\text {Aut }}\left(y_{t}\right) \leq w_{t}\left(w_{0}, y^{t}\right) \leq \bar{w} \quad$ all $t \geq 1$

$(\mathrm{A} 11 \mathrm{~b}) \quad w_{0} \geq U_{0}^{A u t}\left(y_{0}\right)$ given

By theorem 4.4 and 4.5 (which are applicable as $V_{R}$ is bounded on $W$ and the sequence $\left\{\hat{w}_{t}\left(w_{0}, y^{t}\right)\right\}_{t=1}^{\infty}$ defined above never leaves $\left.W\right)$, the allocation $\left\{\hat{h}_{t}\left(w_{0}, y^{t}\right)\right\}_{t=0}^{\infty}$ together with $\left\{\hat{w}_{t}\left(w_{0}, y^{t}\right)\right\}_{t=1}^{\infty}$ defined above uniquely attains the minimum of the above problem. In order to argue that $\left\{\hat{h}_{t}\left(w_{0}, y^{t}\right)\right\}_{t=0}^{\infty}$ solves CPP we have to show that any allocation $\left\{h_{t}\left(w_{0}, y^{t}\right)\right\}_{t=0}^{\infty}$ together with some $\left\{w_{t}\left(w_{0}, y^{t}\right)\right\}_{t=1}^{\infty}$ satisfies A10 and A11a if and only if $\left\{h_{t}\left(w_{0}, y^{t}\right)\right\}_{t=0}^{\infty}$ satisfies 
5,6 and 7 , i.e. if

$(\mathrm{A} 12 \mathrm{c}) \lim _{t \rightarrow \infty} \beta^{t} \sup _{y^{t}} U_{t}\left(w_{0}, y^{t}, \sigma\right)=0$

Step 1: Pick any allocation $\sigma=\left\{h_{t}\left(w_{0}, y^{t}\right)\right\}_{t=0}^{\infty}$ that satisfies A12a to A12c. Define $w_{t}\left(w_{0}, y^{t}\right)=U_{t}\left(w_{0}, y^{t}, \sigma\right)$. It is immediate from A12b that A11a is satisfied. From the definition of $U_{t}\left(w_{0}, y^{t}, \sigma\right)$ it follows that A10 is satisfied as well.

Step 2: Pick any allocation $\sigma=\left\{h_{t}\left(w_{0}, y^{t}\right)\right\}_{t=0}^{\infty}$ and $\left\{w_{t}\left(w_{0}, y^{t}\right)\right\}_{t=1}^{\infty}$ that satisfies A10 and A11a. Since for all $t, w_{t}\left(w_{0}, y^{t}\right) \leq \bar{w}$ from A11a, by using A10 we see that the allocation satisfies A12c. Now for all allocations satisfying A12c, and for all $t$

$$
\begin{aligned}
\left|w_{t}\left(w_{0}, y^{t}\right)-U_{t}\left(w_{0}, y^{t}, \sigma\right)\right| & =\beta\left|\sum_{y^{t+1}} \pi\left(y_{t+1}\right)\left(w_{t+1}\left(w_{0}, y^{t+1}\right)-U_{t}\left(w_{0}, y^{t}, \sigma\right)\right)\right| \\
& \leq \beta \sup _{y^{t+1}}\left|w_{t+1}\left(w_{0}, y^{t+1}\right)-U_{t}\left(w_{0}, y^{t}, \sigma\right)\right| \\
& \leq \beta^{s} \sup _{y^{t+s}}\left|w_{t+s}\left(w_{0}, y^{t+s}\right)-U_{t+s}\left(w_{0}, y^{t+s}, \sigma\right)\right| \\
& \leq \beta^{s} \sup _{y^{t+s}}\left(\left|w_{t+s}\left(w_{0}, y^{t+s}\right)\right|+\left|U_{t+s}\left(w_{0}, y^{t+s}, \sigma\right)\right|\right)
\end{aligned}
$$

This inequality is valid for all $t$ and all $s$. Taking limit with respect to $s$ yields (by A12c and A11a) that $w_{t}\left(w_{0}, y^{t}\right)=U_{t}\left(w_{0}, y^{t}, \sigma\right)$ for all $t$. Hence A10 implies that

$$
\begin{aligned}
w_{0} & =(1-\beta) h_{0}\left(w_{0}, y_{0}\right)+\beta \sum_{y^{1}} \pi\left(y_{1}\right) w_{1}\left(w_{0}, y^{1}\right) \\
& =(1-\beta) h_{0}\left(w_{0}, y_{0}\right)+\beta \sum_{y^{1}} \pi\left(y_{1}\right) U_{1}\left(w_{0}, y^{1}, \sigma\right) \\
& =U_{0}\left(w_{0}, y_{0}, \sigma\right)
\end{aligned}
$$

and hence A12a is satisfied. For $t \geq 1 \mathrm{~A} 12 \mathrm{~b}$ is obviously satisfied, and it is satisfied for $t=0$ by the assumption that $w_{0} \geq U_{t}^{A u t}\left(y_{t}\right)$.

This proves that the allocation constructed from the policies of the functional equation solves the component planning problem with the additional constraint $U_{t}\left(w_{0}, y^{t}, \sigma\right) \leq \bar{w}$. By 
Theorem 2. $\hat{w}_{t}\left(w_{0}, y^{t}\right)<\bar{w}$ and hence, as $\hat{w}_{t}\left(w_{0}, y^{t}\right)=U_{t}\left(w_{0}, y^{t}, \hat{\sigma}\right)$, the constraint is never binding. Since the constraint set associated with the CPP is convex, this implies that the allocation $\hat{\sigma}$ indeed solves the original component planning problem for constant interest rates.

\section{Proof of Lemma 9.:}

Suppose $g_{y_{\max }}(w)>U^{A u t}\left(y_{\max }\right)$ for all $w \in A, w>U^{A u t}\left(y_{\max }\right)$. Then by Lemma 7 . $g_{y^{\prime}}(w)=g_{y_{\max }}(w)$, for all $y^{\prime} \in Y$ and $w>U^{A u t}\left(y_{\max }\right)$. By continuity of $g_{y^{\prime}}$ and Lemma 8 . $g_{y^{\prime}}\left(U^{A u t}\left(y_{\max }\right)\right)=U^{A u t}\left(y_{\max }\right)$, for all $y^{\prime} \in Y$. But since $U^{A u t}\left(y_{\max }\right)>U^{A u t}\left(y^{\prime}\right)$ for all $y^{\prime} \neq y_{\max }$, by Lemma 8. $g_{y^{\prime}}\left(U^{A u t}\left(y_{\max }\right)\right)<U^{A u t}\left(y_{\max }\right)$ for all $y^{\prime} \neq y_{\max }$, a contradiction

\section{Proof of Theorem 4.:}

The proof is an application of Stokey et al., theorem 11.12. We have to prove that there exists an $\varepsilon>0$ and an $N \geq 1$ such that for all sets $(\mathcal{B}, \mathcal{Y}) \in \mathcal{B}(W) \times \mathcal{P}(Y)$, either $Q^{N}\left((w, y, \mathcal{B}, \mathcal{Y}) \geq \varepsilon\right.$ or $Q^{N}\left(\left(w, y,(\mathcal{B}, \mathcal{Y})^{C}\right) \geq \varepsilon\right.$, for all $(w, y) \in(W, Y)$. For this it is sufficient to prove that there exists an $\varepsilon>0$ and an $N \geq 1$ such that for all $(w, y) \in(W, Y)$, $Q^{N}\left(\left(w, y, U^{A u t}\left(y_{\max }\right), y_{\max }\right) \geq \varepsilon\right.$. If $w^{*} \geq \bar{w}$ this is immediate, as for all $(w, y) \in(W, Y)$, $Q\left(\left(w, y, U^{A u t}\left(y_{\max }\right), y_{\max }\right) \geq \pi\left(y_{\max }\right)\right.$, since $g_{y_{\max }}(w)=U^{A u t}\left(y_{\max }\right)$ for all $w \in W$.

Suppose $w^{*}<\bar{w}$. Define

$$
d=\min _{w \in\left[w^{*}, \bar{w}\right]} w-g_{y_{\max }}(w)
$$

Note that $d$ is well-defined as $g_{y_{\max }}$ is a continuous function and that $d>0$ from Lemma 8 . Define

$$
N=\min \left\{n \in N \mid \bar{w}-n d \leq w^{*}\right\}
$$

and $\varepsilon=\pi\left(y_{\max }\right)^{N}$. We will show that for $N$ and $\varepsilon$ so defined the result follows. Suppose an individual receives $y_{\max }$ for $N$ times in a row, an event that occurs with probability $\varepsilon$. For $(w, y)$ such that $w \leq w^{*}$ the result is immediate as for those $w, g_{y_{\max }}(w)=U^{A u t}\left(y_{\max }\right)$ and $g_{y_{\max }}\left(U^{A u t}\left(y_{\max }\right)\right)=U^{A u t}\left(y_{\max }\right)$. For any $w \in\left(w^{*}, \bar{w}\right]$ we have $g_{y_{\max }}(w) \leq w-d$, 
$g_{y_{\max }}\left(g_{y_{\max }}(w)\right) \leq w-2 d$, and so on. Then the result follows by construction of $N$ and $\varepsilon$

\section{Proof of Lemma 10.:}

we have to show that

$$
\lim _{n \rightarrow \infty}\left\|V_{R_{n}}-V_{R}\right\|=0
$$

where ||$V_{R_{n}}-V_{R}||=\sup _{[\underline{w}, \bar{w}]}\left|V_{R_{n}}-V_{R}\right|$. By the triangle inequality

$$
\left\|V_{R_{n}}-V_{R}\right\| \leq\left\|V_{R_{n}}-T_{R_{n}}^{n} V_{R}\right\|+\left\|T_{R_{n}}^{n} V_{R}-V_{R}\right\|
$$

Now the operator $T_{R_{n}}$ is a contraction mapping on $[\underline{w}, \bar{w}]$ with unique fixed point $V_{R_{n}}$ (see corollary 1). Hence

$$
\lim _{n \rightarrow \infty}\left\|V_{R_{n}}-T_{R_{n}}^{n} V_{R}\right\|=0
$$

For the second term in the sum we note that

$$
\left\|T_{R_{n}}^{n} V_{R}-V_{R}\right\| \leq \sum_{k=1}^{n}\left\|T_{R_{n}}^{k} V_{R}-T_{R_{n}}^{k-1} V_{R}\right\| \leq \sum_{k=1}^{n} \frac{1}{\left(R_{n}\right)^{k}}\left\|T_{R_{n}} V_{R}-V_{R}\right\|
$$

Here the first inequality again follows from the triangle inequality and the second from the fact that $T_{R_{n}}$ is a contraction mapping on $[\underline{w}, \bar{w}]$ with modulus $\frac{1}{\left(R_{n}\right)^{k}}$. Hence

$$
\begin{aligned}
\lim _{n \rightarrow \infty}\left\|T_{R_{n}}^{n} V_{R}-V_{R}\right\| & \leq \lim _{n \rightarrow \infty} \sum_{k=1}^{n} \frac{1}{\left(R_{n}\right)^{k}}\left\|T_{R_{n}} V_{R}-V_{R}\right\| \\
& \leq \lim _{n \rightarrow \infty} \frac{R_{n}}{R_{n}-1}\left\|T_{R_{n}} V_{R}-V_{R}\right\| \\
& =\frac{R}{R-1} \lim _{n \rightarrow \infty}\left\|T_{R_{n}} V_{R}-T_{R} V_{R}\right\|
\end{aligned}
$$


where we used the fact that $V_{R}$ is the unique fixed point of $T_{R}$. Hence $\lim _{n \rightarrow \infty}\left\|T_{R_{n}}^{n} V_{R}-V_{R}\right\|=$ 0 if and only if $\lim _{n \rightarrow \infty}\left\|T_{R_{n}} V_{R}-T_{R} V_{R}\right\|=0$, i.e. if the operator $T_{R_{n}}$ is continuous in $R_{n}$. To see that $T_{R_{n}}$ is in fact continuous in $R_{n}$ consider the following argument: for arbitrary $\hat{w} \in[\underline{w}, \bar{w}]$ by the theorem of the maximum

$$
\lim _{n \rightarrow \infty}\left|T_{R_{n}} V_{R}(\hat{w})-T_{R} V_{R}(\hat{w})\right|=0
$$

Since $[\underline{w}, \bar{w}]$ is a compact set and $T_{R_{n}} V_{R}, T_{R} V_{R}$ are continuous functions in $w$, we have

$$
\lim _{n \rightarrow \infty} \max _{\hat{w} \in[\underline{w}, \bar{w}]}\left|T_{R_{n}} V_{R}(\hat{w})-T_{R} V_{R}(\hat{w})\right|=\lim _{n \rightarrow \infty}|| T_{R_{n}} V_{R}-T_{R} V_{R} \|=0
$$

Hence both terms on the right hand side of A15 converge to 0, which proves the result

\section{Proof of Lemma 11.:}

We have to show that for each $\varepsilon>0$ there exists $N(\varepsilon)$ such that for all $n \geq N(\varepsilon)$,

$$
\left|g_{y^{\prime}}^{R_{n}}\left(w_{n}\right)-g_{y^{\prime}}^{R}(w)\right|<\varepsilon
$$

We note that by the triangle inequality

$$
\left|g_{y^{\prime}}^{R_{n}}\left(w_{n}\right)-g_{y^{\prime}}^{R}(w)\right| \leq\left|g_{y^{\prime}}^{R}\left(w_{n}\right)-g_{y^{\prime}}^{R}\left(w_{n}\right)\right|+\left|g_{y^{\prime}}^{R}\left(w_{n}\right)-g_{y^{\prime}}^{R}(w)\right|
$$

Since the function $g_{y^{\prime}}^{R}$ is continuous, for each $\varepsilon_{1}>0$ there exists $N\left(\varepsilon_{1}\right)$ such that $\mid g_{y^{\prime}}^{R}\left(w_{n}\right)-$ $g_{y^{\prime}}^{R}(w) \mid<\varepsilon_{1}$ for all $n \geq N\left(\varepsilon_{1}\right)$. By Lemma 2. $V_{R}$ as well $V_{R_{n}}$ are strictly convex, for each $n \in N$. Also $\left\{V_{R_{n}}\right\}_{n=0}^{\infty}$ converges uniformly to $V_{R}$ by Lemma 10 . on the compact set $[\underline{w}, \bar{w}]$. Then by theorem 3.8, Stokey et al. (1989), for each $\varepsilon_{2}>0$ there exists $N\left(\varepsilon_{2}\right)$ such that $\left|g_{y^{\prime}}^{R_{n}}(w)-g_{y^{\prime}}^{R}(w)\right|<\varepsilon_{2}$ for all $m \geq N\left(\varepsilon_{2}\right)$ and all $w \in[\underline{w}, \bar{w}]$. So fix $\varepsilon>0$ and choose 
$\varepsilon_{1}=\varepsilon_{2}=\frac{\varepsilon}{2}$ and $N(\varepsilon)=\max \left\{N\left(\varepsilon_{1}\right), N\left(\varepsilon_{2}\right)\right\}$. Then for all $n \geq N$

$$
\left|g_{y^{\prime}}^{R_{n}}\left(w_{n}\right)-g_{y^{\prime}}^{R}(w)\right| \leq\left|g_{y^{\prime}}^{R_{n}}\left(w_{n}\right)-g_{y^{\prime}}^{R}\left(w_{n}\right)\right|+\left|g_{y^{\prime}}^{R}\left(w_{n}\right)-g_{y^{\prime}}^{R}(w)\right|<\varepsilon_{2}+\varepsilon_{1}=\varepsilon
$$

\section{Proof of Theorem 5.:}

Consider a sequence $\left\{R_{n}\right\}_{n=0}^{\infty}$ with $R_{n} \in\left(1, \frac{1}{\beta}\right)$ converging to $R \in\left(1, \frac{1}{\beta}\right)$. With each $R_{n}$ and with $R$ there is associated an operator $T_{R_{n}}^{*}$ and $T_{R}^{*}$, respectively. By Theorem 4 . there exist a unique sequence of probability measures $\left\{\Phi_{R_{n}}\right\}_{n=0}^{\infty}$ such that $\Phi_{R_{n}}=T_{R_{n}}^{*} \Phi_{R_{n}}$ and a unique $\Phi_{R}$ such that $\Phi_{R}=T_{R}^{*} \Phi_{R}$. we will argue that the sequence $\left\{\Phi_{R_{n}}\right\}_{n=0}^{\infty}$ converges weakly to $\Phi_{R}$.

First, the state space $[\underline{w}, \bar{w}] \times Y$ is compact. Now consider the sequence of transition functions $\left\{Q_{R_{n}}\right\}_{n=0}^{\infty}$ associated with $\left\{R_{n}\right\}_{n=0}^{\infty}$. For any sequence $\left\{w_{n}\right\}_{n=0}^{\infty}$ in $[\underline{w}, \bar{w}]$ converging to $w \in[\underline{w}, \bar{w}]$, for all $y^{\prime} \in Y, g_{y^{\prime}}^{R_{n}}\left(w_{n}\right)$ converges to $g_{y^{\prime}}(w)$ by Lemma 11 .. Now consider the sequence of probability measures $\left\{Q_{R_{n}}\left(\left(w_{n}, y\right), .\right)\right\}_{n=0}^{\infty}$ and the probability measure $Q_{R}((w, y),$.$) .$ If we can show that for each set $B \in \mathcal{B}(W) \times \mathcal{P}(Y)$ for which $Q_{R}((w, y), \partial B)=0$,

$$
\lim _{n \rightarrow \infty} Q_{R_{n}}\left(\left(w_{n}, y\right), B\right)=Q_{R}((w, y), B)
$$

then the sequence $Q_{R_{n}}\left(\left(w_{n}, y\right),.\right)$ converges weakly to $Q_{R}((w, y),$.$) by theorem 12.3$, Stokey et al. Here $\partial B$ denote the boundary of $B$, i.e.. the set of points that are limit points of $B$ as well as $B^{C}$. Take an arbitrary such set $B$. By definition of $Q_{R}$, for all $w^{\prime}$ such that $g_{y^{\prime}}^{R}(w)=w^{\prime}$ for some $y^{\prime} \in Y$, we have that $w^{\prime}$ is in the interior of $B$ (otherwise $Q_{R}((w, y), \partial B)>0$ ). But then, since $g_{y^{\prime}}^{R_{n}}\left(w_{n}\right)$ converges to $g_{y^{\prime}}(w), Q_{R_{n}}\left(\left(w_{n}, y\right), B\right)=Q_{R}((w, y), B)$ for $n$ sufficiently big. Hence A18 is satisfied and the sequence $Q_{R_{n}}\left(\left(w_{n}, y\right),.\right)$ converges weakly to $Q_{R}((w, y),$.$) .$

This result enables us to apply theorem 12.13 of Stokey et al. to conclude that the sequence $\left\{\Phi_{R_{n}}\right\}_{n=0}^{\infty}$ converges weakly to $\Phi_{R}$. By Lemma 10. $\left\{V_{R_{n}}\right\}_{n=0}^{\infty}$ converges uniformly to 
$V_{R}$. To show continuity of $d($.$) we note that$

$$
\begin{aligned}
\left|d\left(R_{n}\right)-d(R)\right| & =\left|\int V_{R_{n}}(w) d \Phi_{R_{n}}-\int V_{R}(w) d \Phi_{R}\right| \\
& \leq\left|\int V_{R_{n}}(w) d \Phi_{R_{n}}-\int V_{R}(w) d \Phi_{R_{n}}\right|+\left|\int V_{R}(w) d \Phi_{R_{n}}-\int V_{R}(w) d \Phi_{R}\right|
\end{aligned}
$$

by the triangle inequality. The first term converges to zero (as $n \rightarrow \infty)$ as $\left\{V_{R_{n}}\right\}_{n=0}^{\infty}$ converges uniformly to $V_{R}$, the second term converges to zero as $\left\{\Phi_{R_{n}}\right\}_{n=0}^{\infty}$ converges weakly to $\Phi_{R}$ and $V_{R}$ is a continuous and bounded function

\section{Proof of Lemma 12.:}

Let $R>\hat{R}$. We want to show that $h^{R}(w) \leq h^{\hat{R}}(w)$ and $g_{y^{\prime}}^{R}(w) \geq g_{y^{\prime}}^{\hat{R}}(w)$, for all $y^{\prime} \in Y$ and all $w \in[\underline{w}, \bar{w}]$. Define the sequence $\left\{V^{n}\right\}_{n=1}^{\infty}$ by $V^{n}=\left(T_{\hat{R}}\right)^{n} V_{R}$. Note that as $V_{R}$ is strictly convex and differentiable (by the argument in the proof to Lemma 4.), so are all $V^{n}$ (by the argument in the proof to Lemma 4.). Let $\left(h^{n}, g_{y^{\prime}}^{n}\right)$ be the optimal policies associated with $V^{n}$, i.e.

$$
V^{n}(w)=\left(1-\frac{1}{R}\right) C\left(h^{n}(w)\right)+\frac{1}{R} \sum_{y^{\prime}} \pi\left(y^{\prime}\right) V^{n-1}\left(g_{y^{\prime}}^{n}(w)\right) .
$$

We prove by induction that for all $n \geq 1$,

(A22) $h^{R}(w) \leq h^{n}(w)$

(A23) $\frac{V_{R}^{\prime}(w)}{R-1} \leq \frac{\left(V^{n}\right)^{\prime}(w)}{\hat{R}-1}$

for all $y^{\prime} \in Y$ and all $w \in[\underline{w}, \bar{w}]$. Since $\left\{V^{n}\right\}_{n=1}^{\infty}$ converges to $V_{\hat{R}}$ uniformly (by corollary 1.) and $\left\{h^{n}, g_{y^{\prime}}^{n}\right\}_{n=1}^{\infty}$ converge uniformly to $\left(h^{\hat{R}}, g_{y^{\prime}}^{\hat{R}}\right)$ (again see Lemma 4.), it then follows that $g_{y^{\prime}}^{R}(w) \geq g_{y^{\prime}}^{\hat{R}}(w)$ (and the other two relations also hold for $n$ replaced with $\hat{R}$ ).

Step 1: Let $n=1$ and fix $w \in[\underline{w}, \bar{w}]$

Suppose, to obtain a contradiction, that there exists $y^{\prime}$ such that $g_{y^{\prime}}^{1}(w)>g_{y^{\prime}}^{R}(w) \geq$ 
$U^{A u t}\left(y^{\prime}\right)$. Then from the respective first order conditions (note that $V^{1}=T_{\hat{R}} V_{R}$ )

(A24) $V_{R}^{\prime}\left(g_{y^{\prime}}^{1}(w)\right)=\frac{\beta(\hat{R}-1)}{1-\beta} C^{\prime}\left(h^{1}(w)\right)$

$(\mathrm{A} 25) V_{R}^{\prime}\left(g_{y^{\prime}}^{R}(w)\right) \geq \frac{\beta(R-1)}{1-\beta} C^{\prime}\left(h^{R}(w)\right)$

Since $V_{R}$ is strictly convex $V_{R}^{\prime}\left(g_{y^{\prime}}^{1}(w)\right)>V_{R}^{\prime}\left(g_{y^{\prime}}^{R}(w)\right)$ and hence (as $\left.R>\hat{R}\right), h^{1}(w)>h^{R}(w)$.

From the promise keeping constraint there must exist $\bar{y}^{\prime}$ such that $g_{\bar{y}^{\prime}}^{R}(w)>g_{\bar{y}^{\prime}}^{1}(w) \geq U^{A u t}\left(\bar{y}^{\prime}\right)$.

But then (using A24 and A25)

$$
V_{R}^{\prime}\left(g_{\bar{y}^{\prime}}^{R}(w)\right)=\frac{\beta(R-1)}{1-\beta} C^{\prime}\left(h^{R}(w)\right)<\frac{\beta(\hat{R}-1)}{1-\beta} C^{\prime}\left(h^{1}(w)\right) \leq V_{R}^{\prime}\left(g_{\bar{y}^{\prime}}^{1}(w)\right)
$$

which implies $g_{\bar{y}^{\prime}}^{R}(w)<g_{\bar{y}^{\prime}}^{1}(w)$, a contradiction. Hence $g_{y^{\prime}}^{1}(w) \leq g_{y^{\prime}}^{R}(w)$, for all $y^{\prime} \in Y$. Then from the promise keeping constraint $h^{1}(w) \geq h^{R}(w)$. The envelope conditions are

$$
\begin{aligned}
\frac{\left(V^{1}\right)^{\prime}(w)}{\hat{R}-1} & =\frac{C^{\prime}\left(h^{1}(w)\right)}{\hat{R}(1-\beta)} \\
\frac{V_{R}^{\prime}(w)}{R-1} & =\frac{C^{\prime}\left(h^{R}(w)\right)}{R(1-\beta)}
\end{aligned}
$$

It follows from the previous result that $\frac{\left(V^{1}\right)^{\prime}(w)}{\hat{R}-1} \geq \frac{V_{R}^{\prime}(w)}{R-1}$.

Step 2: Suppose that A21 to A23 are true for $n-1$. We want to show that A21 to A23 are true for $n$. Again suppose, to obtain a contradiction, that there exists $y^{\prime}$ such that $g_{y^{\prime}}^{n}(w)>g_{y^{\prime}}^{R}(w) \geq U^{A u t}\left(y^{\prime}\right)$. From the first order conditions we have

$$
\begin{aligned}
\frac{\left(V^{n-1}\right)^{\prime}\left(g_{y^{\prime}}^{n}(w)\right)}{\hat{R}-1} & =\frac{\beta}{1-\beta} C^{\prime}\left(h^{n}(w)\right) \\
\frac{V_{R}^{\prime}\left(g_{y^{\prime}}^{R}(w)\right)}{R-1} & \geq \frac{\beta}{1-\beta} C^{\prime}\left(h^{R}(w)\right)
\end{aligned}
$$

Since $V_{R}$ and $V^{n-1}$ are convex, $g_{y^{\prime}}^{n}(w)>g_{y^{\prime}}^{R}(w)$ and A23 holds for $n-1$, we have that $h^{n}(w)>h^{R}(w)$. Again by the promise keeping constraints there exists $\bar{y}^{\prime}$ such that $g_{\bar{y}^{\prime}}^{R}(w)>$ 
$g_{\bar{y}^{\prime}}^{n}(w) \geq U^{A u t}\left(\bar{y}^{\prime}\right)$. But by Lemma 7 .

$$
\begin{aligned}
& g_{y^{\prime}}^{n}(w) \leq g_{\bar{y}^{\prime}}^{n}(w) \\
& g_{y^{\prime}}^{R}(w) \geq g_{\bar{y}^{\prime}}^{R}(w)
\end{aligned}
$$

and hence

$$
g_{y^{\prime}}^{n}(w) \leq g_{\bar{y}^{\prime}}^{n}(w)<g_{\bar{y}^{\prime}}^{R}(w) \leq g_{y^{\prime}}^{R}(w)<g_{y^{\prime}}^{n}(w)
$$

a contradiction. It follows that for all $y^{\prime} \in Y, g_{y^{\prime}}^{n}(w) \leq g_{y^{\prime}}^{R}(w)$. From promise keeping we have $h^{n}(w) \geq h^{R}(w)$. As before the envelope conditions imply that $\frac{\left(V^{n}\right)^{\prime}(w)}{\hat{R}-1} \geq \frac{V_{R}^{\prime}(w)}{R-1}$

\section{Proof of Lemma 13.:}

Define the sequence of measures $\left\{\Phi_{n}\right\}_{n=1}^{\infty}$ by $\Phi_{n}=\left(T_{\hat{R}}^{*}\right)^{n} \Phi_{R}$. We shall prove by induction that for each $n \geq 1$, and each $y \in Y, \Phi_{R}^{y}$ stochastically dominates $\Phi_{n}^{y}$. Since by Theorem 4. $\left\{\Phi_{n}\right\}$ converges to $\Phi_{\hat{R}}$ in total variation norm, the result then follows.

It will be convenient to define the distribution function associated with any probability measure $\Phi_{n}^{y}, F_{n}^{y}: W \rightarrow[0,1]$, as $F_{n}^{y}(w)=\Phi_{n}^{y}([\underline{w}, w])=\Phi_{n}([\underline{w}, w],\{y\}) / \pi(y)$. Since the domain of these functions is a subset of $\Re^{1}$, in order to prove that $\Phi_{R}^{y}$ stochastically dominates $\Phi_{n}^{y}$ it is sufficient to prove that for all $w \in W, F_{R}^{y}(w) \leq F_{n}^{y}(w)$.

Step 1: Let $n=1$

By definition $\Phi_{1}=T_{\hat{R}}^{*} \Phi_{R}$ whereas $\Phi_{R}=T_{R}^{*} \Phi_{R}$. Fix an arbitrary $y \in Y, w \in W$. Then

$$
\begin{aligned}
F_{R}^{y}(w) & =\frac{\Phi_{R}([\underline{w}, w],\{y\})}{\pi(y)} \\
& =\int_{\left\{v \in W \mid g_{y}^{R}(v) \leq w\right\}} d \Phi_{R}^{y} \\
& \leq \int_{\left\{v \in W \mid g_{y}^{\hat{R}}(v) \leq w\right\}} d \Phi_{R}^{y} \\
& =\frac{\Phi_{1}([\underline{w}, w],\{y\})}{\pi(y)} \\
& =F_{1}^{y}(w)
\end{aligned}
$$

where the inequality is due to the fact that $g_{y}^{R}(w) \geq g_{y}^{\hat{R}}(w)$, for all $w \in W$. 
Step 2: Suppose $F_{R}^{y}(w) \leq F_{n-1}^{y}(w)$, for all $w \in W$, all $y \in Y$. We want to show that the same is true for $n$. Note that

$$
\begin{aligned}
F_{n}^{y}(w) & =\frac{\Phi_{n}([\underline{w}, w],\{y\})}{\pi(y)} \\
& =\int_{\left\{v \in W \mid g_{y}^{\hat{R}}(v) \leq w\right\}} d \Phi_{n-1}^{y} \\
& =\sum_{\bar{y} \in Y} \pi(\bar{y}) F_{n-1}^{\bar{y}}\left(v_{n}\right)
\end{aligned}
$$

where $v_{n}:=\max \left\{v \in W \mid g_{y}^{\hat{R}}(v) \leq w\right\}$. Note that the last equality requires $g_{y}^{\hat{R}}$ to be increasing in $v$ as shown in Lemma 6. Continuity of $g_{y}^{\hat{R}}$ ensures that $v_{n}$ is well-defined. Similarly $F_{R}^{y}(w)=\sum_{\bar{y} \in Y} \pi(\bar{y}) F_{R}^{\bar{y}}\left(v_{R}\right)$ with $v_{R}:=\max \left\{v \in W \mid g_{y}^{R}(v) \leq w\right\}$. Lemma 12. implies that $v_{R} \leq v_{n}$. Then the induction hypothesis implies that for all $\bar{y} \in Y, F_{R}^{\bar{y}}\left(v_{R}\right) \leq F_{n-1}^{\bar{y}}\left(v_{n}\right)$, and hence $F_{R}^{y}(w) \leq F_{n}^{y}(w)$

\section{Proof of Theorem 6.:}

By definition of $d(R)$

$$
d(R)=\int V_{R}(w) d \Phi_{R}-\int y d \Phi_{R}
$$

Since for all $R, \int y d \Phi_{R}$ is a constant, we focus on the analysis of the first part of the excess demand function. From the functional equation

$$
\int V_{R}(w) d \Phi_{R}=\left(1-\frac{1}{R}\right) \int C\left(h^{R}(w)\right) d \Phi_{R}+\frac{1}{R} \sum_{y^{\prime}} \pi\left(y^{\prime}\right) \int V\left(g_{y^{\prime}}^{R}(w)\right) d \Phi_{R}
$$

we note that by stationarity and the definition of $\Phi_{R}^{y}$,

$$
\begin{aligned}
\int V_{R}(w) d \Phi_{R} & =\sum_{y \in Y} \pi(y) \int V_{R}(w) d \Phi_{R}^{y} \\
\int V\left(g_{y^{\prime}}^{R}(w)\right) d \Phi_{R} & =\int V(w) d \Phi_{R}^{y^{\prime}}
\end{aligned}
$$


so that

(A32)

$$
\int V_{R}(w) d \Phi_{R}=\sum_{y^{\prime}} \pi\left(y^{\prime}\right) \int V\left(g_{y^{\prime}}^{R}(w)\right) d \Phi_{R}
$$

It follows that

$$
\int V_{R}(w) d \Phi_{R}=\int C\left(h^{R}(w)\right) d \Phi_{R}
$$

We want to prove that

$$
\int V_{R}(w) d \Phi_{R} \geq \int V_{\hat{R}}(w) d \Phi_{\hat{R}}
$$

By the previous lemma for all $y \in Y, \Phi_{R}^{y}$ stochastically dominates $\Phi_{\hat{R}}^{y}$, and since $V_{\hat{R}}$ is strictly increasing it follows (using A30 that

(A34)

$$
\int V_{\hat{R}}(w) d \Phi_{R} \geq \int V_{\hat{R}}(w) d \Phi_{\hat{R}}
$$

So if we can prove that

$$
\int V_{R}(w) d \Phi_{R} \geq \int V_{\hat{R}}(w) d \Phi_{R}
$$

we are done. Define the sequence $\left\{V^{n}\right\}_{n=1}^{\infty}$ by $V^{n}=\left(T_{\hat{R}}\right)^{n} V_{R}$. We will prove by induction that for all $n \geq 1$

$$
\int V_{R}(w) d \Phi_{R} \geq \int V^{n}(w) d \Phi_{R}
$$


Since the sequence $\left\{V^{n}\right\}_{n=1}^{\infty}$ converges uniformly to $V_{\hat{R}}$ (by corollary 1.), this proves A35.

Let $\left\{h^{n}, g_{y^{\prime}}^{n}\right\}_{n=1}^{\infty}$ be the optimal policies associated with $\left\{V^{n}\right\}_{n=1}^{\infty}$ and $\left(h^{R}, g_{y^{\prime}}^{R}\right)$ be the optimal choices associated with $V_{R}$.

Step 1: Let $n=1$.

By definition $V^{1}=T_{\hat{R}} V_{R}$. Hence

$$
\begin{aligned}
V^{1}(w) & =\left(1-\frac{1}{\hat{R}}\right) C\left(h^{1}(w)\right)+\frac{1}{\hat{R}} \sum \pi\left(y^{\prime}\right) V_{R}\left(g_{y^{\prime}}^{1}(w)\right) \\
& \leq\left(1-\frac{1}{\hat{R}}\right) C\left(h^{R}(w)\right)+\frac{1}{\hat{R}} \sum \pi\left(y^{\prime}\right) V_{R}\left(g_{y^{\prime}}^{R}(w)\right)
\end{aligned}
$$

since $\left(h^{1}, g_{y^{\prime}}^{1}\right)$ are the minimizing choices associated with $V^{1}$. Integrating with respect to $\Phi_{R}$ and using A33 and A32 yields

$$
\begin{aligned}
\int V^{1}(w) d \Phi_{R} & =\left(1-\frac{1}{\hat{R}}\right) \int C\left(h^{1}(w)\right) d \Phi_{R}+\frac{1}{\hat{R}} \sum \pi\left(y^{\prime}\right) \int V_{R}\left(g_{y^{\prime}}^{1}(w)\right) d \Phi_{R} \\
& \leq\left(1-\frac{1}{\hat{R}}\right) \int C\left(h^{R}(w)\right) d \Phi_{R}+\frac{1}{\hat{R}} \sum \pi\left(y^{\prime}\right) \int V_{R}\left(g_{y^{\prime}}^{R}(w)\right) d \Phi_{R} \\
& =\left(1-\frac{1}{\hat{R}}\right) \int V_{R}(w) d \Phi_{R}+\frac{1}{\hat{R}} \sum \pi\left(y^{\prime}\right) \int V_{R}\left(g_{y^{\prime}}^{R}(w)\right) d \Phi_{R} \\
& =\int V_{R}(w) d \Phi_{R}
\end{aligned}
$$

Step 2: Suppose $\int V_{R}(w) d \Phi_{R} \geq \int V^{n-1}(w) d \Phi_{R}$. We want to show that the same is true for $n$. By definition $V^{n}=T_{\hat{R}} V^{n-1}$, hence

$$
\begin{aligned}
V^{n}(w) & =\left(1-\frac{1}{\hat{R}}\right) C\left(h^{n}(w)\right)+\frac{1}{\hat{R}} \sum \pi\left(y^{\prime}\right) V^{n-1}\left(g_{y^{\prime}}^{n}(w)\right) \\
& \leq\left(1-\frac{1}{\hat{R}}\right) C\left(h^{R}(w)\right)+\frac{1}{\hat{R}} \sum \pi\left(y^{\prime}\right) V^{n-1}\left(g_{y^{\prime}}^{R}(w)\right)
\end{aligned}
$$

by the same reason as in step 1 . Again integrating with respect to $\Phi_{R}$ and using A33 and 
A32 we obtain

$$
\begin{aligned}
\int V^{n}(w) d \Phi_{R} & =\left(1-\frac{1}{\hat{R}}\right) \int C\left(h^{n}(w)\right) d \Phi_{R}+\frac{1}{\hat{R}} \sum \pi\left(y^{\prime}\right) \int V^{n-1}\left(g_{y^{\prime}}^{n}(w)\right) d \Phi_{R} \\
& \leq\left(1-\frac{1}{\hat{R}}\right) \int C\left(h^{R}(w)\right) d \Phi_{R}+\frac{1}{\hat{R}} \sum \pi\left(y^{\prime}\right) \int V^{n-1}\left(g_{y^{\prime}}^{R}(w)\right) d \Phi_{R} \\
& =\left(1-\frac{1}{\hat{R}}\right) \int V_{R}(w) d \Phi_{R}+\frac{1}{\hat{R}} \int V^{n-1}(w) d \Phi_{R} \\
& \leq\left(1-\frac{1}{\hat{R}}\right) \int V_{R}(w) d \Phi_{R}+\frac{1}{\hat{R}} \int V_{R}(w) d \Phi_{R} \\
& =\int V_{R}(w) d \Phi_{R}
\end{aligned}
$$

where the last inequality uses the induction hypothesis

\section{Proof of Theorem 7.}

It is obvious that the allocation satisfies the resource constraint 33 since the efficient allocation by construction satisfies the resource constraint, and $\Theta_{0}$ is derived from $\Phi_{0}$. Also the allocation satisfies the continuing participation constraints, and, by construction of $a_{0}\left(w_{0}, y_{0}\right)$, the budget constraint. So it remains to be shown that, for almost all $\left(a_{0}, y_{0}\right),\left\{c_{t}\left(a_{0}, y^{t}\right)\right\}$ is utility maximizing among the allocations satisfying the budget and the continuing participation constraints.

The proof is in two steps. We first show that the first order conditions 34 are sufficient for optimality and we then show that the allocation defined above indeed satisfies the first order conditions.

Step 1. Define

$$
\begin{aligned}
& U\left(a_{0}, y^{t}\right)=(1-\beta) u\left(c_{t}\left(a_{0}, y^{t}\right)\right)+\sum_{s>t} \sum_{y^{s} \mid y^{t}} \beta^{s-t} \pi\left(y^{s} \mid y^{t}\right)(1-\beta) u\left(c_{s}\left(a_{0}, y^{s}\right)\right) \\
& U^{A u t}\left(y_{t}\right)=(1-\beta) u\left(y_{t}\right)+\sum_{s>t} \sum_{y^{s} \mid y^{t}} \beta^{s-t} \pi\left(y^{s} \mid y^{t}\right)(1-\beta) u\left(y_{s}\right)
\end{aligned}
$$

Suppose there exist Lagrange multipliers $\lambda\left(a_{0}, y_{0}\right),\left\{\mu\left(a_{0}, y^{t}\right)\right\} \geq 0$ that jointly with $\left\{c_{t}\left(a_{0}, y^{t}\right)\right\}$ satisfy 34 , the budget constraint 31 (at prices defined in 38) as well as the continuing partic- 
ipation constraints 32

$$
U\left(a_{0}, y^{t}\right) \geq U^{A u t}\left(y_{t}\right)
$$

together with

$$
\mu\left(a_{0}, y^{t}\right)\left(U\left(a_{0}, y^{t}\right)-U^{A u t}\left(y_{t}\right)\right)=0 .
$$

Now suppose that there is a consumption allocation for individuals of type $\left(a_{0}, y_{0}\right),\left\{\hat{c}_{t}\left(a_{0}, y^{t}\right)\right\}$, that satisfies 31 and 32 , and that dominates $\left\{c_{t}\left(a_{0}, y^{t}\right)\right\}$, i.e. $\hat{U}\left(a_{0}, y_{0}\right)>U\left(a_{0}, y_{0}\right)$, where 
$\hat{U}\left(a_{0}, y_{0}\right)$ is defined analogously to A40. Then

$$
0<\hat{U}\left(a_{0}, y_{0}\right)-U\left(a_{0}, y_{0}\right)
$$

$(\mathrm{A} 43)$

$$
=(1-\beta)\left(\sum_{t=0}^{\infty} \sum_{y^{t} \mid y_{0}} \beta^{t} \pi\left(y^{t} \mid y_{0}\right)\left[u\left(\hat{c}_{t}\left(a_{0}, y^{t}\right)\right)-u\left(c_{t}\left(a_{0}, y^{t}\right)\right)\right]\right)
$$

$$
\leq(1-\beta)\left(\sum_{t=0}^{\infty} \sum_{y^{t} \mid y_{0}} \beta^{t} \pi\left(y^{t} \mid y_{0}\right)\left[1+\sum_{y^{\tau} \in P\left(y^{t}\right)} \mu\left(a_{0}, y^{\tau}\right)\right]\left[u\left(\hat{c}_{t}\left(a_{0}, y^{t}\right)\right)-u\left(c_{t}\left(a_{0}, y^{t}\right)\right)\right]\right)
$$

$$
<(1-\beta)\left(\sum_{t=0}^{\infty} \sum_{y^{t} \mid y_{0}} \beta^{t} \pi\left(y^{t} \mid y_{0}\right)\left[1+\sum_{y^{\tau} \in P\left(y^{t}\right)} \mu\left(a_{0}, y^{\tau}\right)\right] u^{\prime}\left(c_{t}\left(a_{0}, y^{t}\right)\right)\left(\hat{c}_{t}\left(a_{0}, y^{t}\right)-c_{t}\left(a_{0}, y^{t}\right)\right)\right)
$$

$$
=\lambda\left(a_{0}, y_{0}\right)\left(\sum_{t=0}^{\infty} \sum_{y^{t} \mid y_{0}} p\left(y^{t}\right)\left(\hat{c}_{t}\left(a_{0}, y^{t}\right)-c_{t}\left(a_{0}, y^{t}\right)\right)\right)
$$

$$
=\lambda\left(a_{0}, y_{0}\right)\left(\sum_{t=0}^{\infty} \sum_{y^{t} \mid y_{0}} p\left(y^{t}\right) \hat{c}_{t}\left(a_{0}, y^{t}\right)-\sum_{t=0}^{\infty} \sum_{y^{t} \mid y_{0}} p\left(y^{t}\right) y_{t}-a_{0}\right)
$$

$$
\leq 0
$$

a contradiction. The several steps in the argument are justified as follows: A43 is by definition, A44 will be proved below, A45 follows from strict concavity of the utility function, A46 follows from 34, A47 from the budget constraint and the fact that $u$ is strictly increasing and prices are strictly positive, and finally A48 follows from the budget constraint. Hence there does not exist a consumption allocation $\left\{\hat{c}_{t}\left(a_{0}, y^{t}\right)\right\}$, that satisfies 31 and 32 , and that dominates $\left\{c_{t}\left(a_{0}, y^{t}\right)\right\}$.

Now we prove that inequality A44 holds. For this we first note that for all $t, y^{t}$, we 
have

(A49)

$$
\left(1+\mu\left(a_{0}, y^{t}\right)\right)\left(\hat{U}\left(a_{0}, y^{t}\right)-U\left(a_{0}, y^{t}\right)\right) \geq \hat{U}\left(a_{0}, y^{t}\right)-U\left(a_{0}, y^{t}\right)
$$

If $U\left(a_{0}, y^{t}\right)>U^{\text {Aut }}\left(y_{t}\right)$, then from A42 it follows that $\mu\left(a_{0}, y^{t}\right)=0$, so that A49 is satisfied. If $U\left(a_{0}, y^{t}\right)=U^{A u t}\left(y_{t}\right)$, then $\hat{U}\left(a_{0}, y^{t}\right) \leq U\left(a_{0}, y^{t}\right)$ and $\mu\left(a_{0}, y^{t}\right) \geq 0$, and again A49 holds.

Now

$$
\begin{aligned}
& \hat{U}\left(a_{0}, y_{0}\right)-U\left(a_{0}, y_{0}\right) \\
\leq & \left(1+\mu\left(a_{0}, y_{0}\right)\right)\left(\hat{U}\left(a_{0}, y_{0}\right)-U\left(a_{0}, y_{0}\right)\right) \\
= & \left(1+\mu\left(a_{0}, y_{0}\right)\right) *(1-\beta)\left(\sum_{t=0}^{\infty} \sum_{y^{t} \mid y_{0}} \beta^{t} \pi\left(y^{t} \mid y_{0}\right)\left[u\left(c_{t}\left(a_{0}, y^{t}\right)\right)-u\left(\hat{c}_{t}\left(a_{0}, y^{t}\right)\right)\right]\right) \\
= & \left(1+\mu\left(a_{0}, y_{0}\right)\right) *(1-\beta)\left[u\left(\hat{c}_{0}\left(a_{0}, y_{0}\right)\right)-u\left(c_{t}\left(a_{0}, y^{t}\right)\right)\right] \\
& +\left(1+\mu\left(a_{0}, y_{0}\right)\right) * \beta \sum_{y_{1}} \pi\left(y_{1} \mid y_{0}\right)\left(\hat{U}\left(a_{0}, y^{1}\right)-U\left(a_{0}, y^{1}\right)\right) \\
\leq & \left(1+\mu\left(a_{0}, y_{0}\right)\right) *(1-\beta)\left[u\left(\hat{c}_{0}\left(a_{0}, y_{0}\right)\right)-u\left(c_{t}\left(a_{0}, y^{t}\right)\right)\right] \\
& +\beta \sum_{y_{1}} \pi\left(y_{1} \mid y_{0}\right)\left(1+\mu\left(a_{0}, y_{0}\right)+\mu\left(a_{0}, y^{1}\right)\right)\left(\hat{U}\left(a_{0}, y^{1}\right)-U\left(a_{0}, y^{1}\right)\right) \\
& \vdots \\
\leq & \sum_{t=0}^{T} \sum_{y^{t} \mid y_{0}} \beta^{t} \pi\left(y^{t} \mid y_{0}\right)\left(1+\sum_{y^{\tau} \in P\left(y^{t}\right)} \mu\left(a_{0}, y^{\tau}\right)\right)\left[u\left(c_{t}\left(a_{0}, y^{t}\right)\right)-u\left(\hat{c}_{t}\left(a_{0}, y^{t}\right)\right)\right] \\
& +\sum_{y^{T} \mid y_{0}} \sum_{y_{T+1}} \beta^{T+1} \pi\left(y^{T+1} \mid y_{0}\right)\left(1+\sum_{y^{\tau} \in P\left(y^{T+1}\right)} \mu\left(a_{0}, y^{\tau}\right)\right)\left(\hat{U}\left(a_{0}, y^{T+1}\right)-U\left(a_{0}, y^{T+1}\right)\right)
\end{aligned}
$$

Taking limits yields

$$
\begin{aligned}
& \hat{U}\left(a_{0}, y_{0}\right)-U\left(a_{0}, y_{0}\right) \\
\leq & (1-\beta)\left(\sum_{t=0}^{\infty} \sum_{y^{t} \mid y_{0}} \beta^{t} \pi\left(y^{t} \mid y_{0}\right)\left[1+\sum_{y^{\tau} \in P\left(y^{t}\right)} \mu\left(a_{0}, y^{\tau}\right)\right]\left[u\left(\hat{c}_{t}\left(a_{0}, y^{t}\right)\right)-u\left(c_{t}\left(a_{0}, y^{t}\right)\right)\right]\right) \\
& +\lim _{T \rightarrow \infty} \sum_{y^{T+1} \mid y_{0}} \beta^{T+1} \pi\left(y^{T+1} \mid y_{0}\right)\left(1+\sum_{y^{\tau} \in P\left(y^{T+1}\right)} \mu\left(a_{0}, y^{\tau}\right)\right)\left(\hat{U}\left(a_{0}, y^{T+1}\right)-U\left(a_{0}, y^{T+1}\right)\right)
\end{aligned}
$$


We need to show that the last limit is nonpositive. Now note that from 34

$$
\begin{aligned}
& \lim _{T \rightarrow \infty} \sum_{y^{T+1} \mid y_{0}} \beta^{T+1} \pi\left(y^{T+1} \mid y_{0}\right)\left(1+\sum_{y^{\tau} \in P\left(y^{T+1}\right)} \mu\left(a_{0}, y^{\tau}\right)\right)\left(\hat{U}\left(a_{0}, y^{T+1}\right)-U\left(a_{0}, y^{T+1}\right)\right) \\
= & \lim _{T \rightarrow \infty} \frac{\lambda\left(a_{0}, y_{0}\right)}{(1-\beta) R^{T+1}} \sum_{y^{T+1} \mid y_{0}} \frac{\pi\left(y^{T+1} \mid y_{0}\right)\left(\hat{U}\left(a_{0}, y^{T+1}\right)-U\left(a_{0}, y^{T+1}\right)\right)}{u^{\prime}\left(c_{T+1}\left(a_{0}, y^{T+1}\right)\right)} \\
= & \frac{\lambda\left(a_{0}, y_{0}\right)}{(1-\beta)} \lim _{T \rightarrow \infty} \sum_{y^{T+1} \mid y_{0}} \frac{\pi\left(y^{T+1} \mid y_{0}\right) \hat{U}\left(a_{0}, y^{T+1}\right)}{R^{T+1} u^{\prime}\left(c_{T+1}\left(a_{0}, y^{T+1}\right)\right)}
\end{aligned}
$$

because, since $\left\{c_{t}\left(a_{0}, y^{t}\right)\right\}$ is bounded, $\lim _{T \rightarrow \infty} \sum_{y^{T+1} \mid y_{0}} \frac{U\left(a_{0}, y^{T+1}\right)}{R^{T+1}}=0$. Now

$$
\begin{aligned}
& \frac{\lambda\left(a_{0}, y_{0}\right)}{(1-\beta)} \lim _{T \rightarrow \infty} \sum_{y^{T+1} \mid y_{0}} \frac{\pi\left(y^{T+1} \mid y_{0}\right) \hat{U}\left(a_{0}, y^{T+1}\right)}{u^{\prime}\left(c_{T+1}\left(a_{0}, y^{T+1}\right)\right) R^{T+1}} \\
\leq & \frac{\lambda\left(a_{0}, y_{0}\right)}{(1-\beta)} \lim _{T \rightarrow \infty} \sum_{s \geq T+1} \sum_{y^{s} \mid y^{T+1}} \frac{\beta^{s-T-1} \pi\left(y^{s} \mid y_{0}\right) u\left(\hat{c}_{s}\left(a_{0}, y^{s}\right)\right)}{u^{\prime}\left(c_{T+1}\left(a_{0}, y^{T+1}\right)\right) R^{T+1}}
\end{aligned}
$$

Without loss of generality we can sum only over those elements for which $u\left(\hat{c}_{s}\left(a_{0}, y^{s}\right)\right)>0$ (it makes the expression only bigger). Then

$$
\begin{aligned}
& \frac{\lambda\left(a_{0}, y_{0}\right)}{(1-\beta)} \lim _{T \rightarrow \infty} \sum_{s \geq T+1} \sum_{y^{s} \mid y^{T+1}} \frac{\beta^{s-T-1} \pi\left(y^{s} \mid y_{0}\right) u\left(\hat{c}_{s}\left(a_{0}, y^{s}\right)\right)}{u^{\prime}\left(c_{T+1}\left(a_{0}, y^{T+1}\right)\right) R^{T+1}} \\
\leq & \frac{\lambda\left(a_{0}, y_{0}\right)}{(1-\beta) u^{\prime}(\bar{c})} \lim _{T \rightarrow \infty} \sum_{s \geq T+1} \sum_{y^{s} \mid y^{T+1}} \frac{\pi\left(y^{s} \mid y_{0}\right) u\left(\hat{c}_{s}\left(a_{0}, y^{s}\right)\right)}{R^{s}}
\end{aligned}
$$

where we used the facts that if we can show that $\left\{c_{t}\left(a_{0}, y^{t}\right)\right\}$ is bounded is bounded above by, say $\bar{c}$, and that $\beta<\frac{1}{R}$. From the budget constraint we know that (given the conjectured equilibrium prices)

$$
\lim _{T \rightarrow \infty} \sum_{s \geq T+1}^{\infty} \sum_{y^{s} \mid y^{T+1}} \frac{\pi\left(y^{s} \mid y_{0}\right) \hat{c}_{t}\left(a_{0}, y^{t}\right)}{R^{s}}=0
$$

Since the utility function satisfies the INADA conditions, there exists $c^{*}>0$ such that $u^{\prime}\left(c^{*}\right)=1$. By concavity $u\left(\hat{c}_{t}\left(a_{0}, y^{t}\right)\right) \leq u\left(c^{*}\right)+u^{\prime}\left(c^{*}\right)\left(\hat{c}_{t}\left(a_{0}, y^{t}\right)-c^{*}\right)$. Hence 


$$
\begin{aligned}
& \lim _{T \rightarrow \infty} \sum_{s \geq T+1}^{\infty} \sum_{y^{s} \mid y^{T+1}} \frac{\pi\left(y^{s} \mid y_{0}\right) u\left(\hat{c}_{t}\left(a_{0}, y^{t}\right)\right)}{R^{s}} \\
\leq & \lim _{T \rightarrow \infty} \sum_{s \geq T+1}^{\infty} \sum_{y^{s} \mid y^{T+1}} \frac{\pi\left(y^{s} \mid y_{0}\right) \hat{c}_{t}\left(a_{0}, y^{t}\right)}{R^{s}}+\left(u\left(c^{*}\right)-c^{*}\right) \lim _{T \rightarrow \infty} \sum_{s \geq T+1}^{\infty} \frac{1}{R^{s}} \\
= & \left(u\left(c^{*}\right)-c^{*}\right) \lim _{T \rightarrow \infty} \sum_{s \geq T+1}^{\infty} \frac{1}{R^{s}} \\
= & \left(u\left(c^{*}\right)-c^{*}\right) \frac{R}{R-1} \lim _{T \rightarrow \infty} \frac{1}{R^{T+1}} \\
= & 0
\end{aligned}
$$

and we are done.

Step 2. We want to show that there exist Lagrange multipliers $\lambda\left(a_{0}, y_{0}\right),\left\{\mu\left(a_{0}, y^{t}\right\} \geq 0\right.$ that, together with the consumption allocation $\left\{c_{t}\left(a_{0}, y^{t}\right)\right\}$ satisfies the first order conditions. Define

$$
\begin{aligned}
& \mu\left(a_{0}, y_{0}\right)=0 \\
& \lambda\left(a_{0}, y_{0}\right)=(1-\beta) u^{\prime}\left(c_{0}\left(a_{0}, y_{0}\right)\right)
\end{aligned}
$$

and recursively

$$
1+\sum_{y^{\tau} \mid y^{t}} \mu\left(a_{0}, y^{\tau}\right)=\frac{u^{\prime}\left(c_{0}\left(a_{0}, y_{0}\right)\right)}{(\beta R)^{t} u^{\prime}\left(c_{t}\left(a_{0}, y^{t}\right)\right)}
$$

Note that the allocation by construction (see A7) satisfies

$$
\frac{u^{\prime}\left(c_{t}\left(a_{0}, y_{0}\right)\right)}{\beta R u^{\prime}\left(c_{t+1}\left(a_{0}, y^{t+1}\right)\right)} \geq 1,
$$

with equality if the limited enforcement constraint is not binding in contingency $y^{t+1}$. Hence $\mu\left(a_{0}, y^{t+1}\right) \geq 0$, and $\mu\left(a_{0}, y^{t+1}\right)=0$ if the constraint is not binding. Obviously the allocation 
and multipliers satisfy 34 - 


\section{A3. The Computational Procedure}

In this subsection we describe how, for a parametric class of our economy, we compute a constant $R$, policy rules $h^{R}(w, y), g_{y^{\prime}}^{R}(w, y)$ and a distribution over utility entitlements and endowment shocks,$\Phi_{R}$ as described in the last section.

Our computational method is an implementation of the policy function iteration algorithm proposed by Coleman (1990). For a fixed $R$ we search for the optimal policies $g_{y^{\prime}}(w, y)$ and $h(w, y)$ within the class of piecewise-linear functions in $w$. We start by specifying a $k$ point grid $G=\left\{w_{0}, \ldots, w_{k}\right\} \subseteq D$ and by guessing the values of a function $V_{0}^{\prime}(.,$.$) on G \times Y$. Notice that this defines a function piecewise linear in $w$ for a fixed $y$. For a given $w, y \in G \times Y$ we then use the first order condition

$$
\begin{aligned}
C^{\prime}(h(w, y)) & \leq \frac{1-\beta}{\beta(R-1)} V^{\prime}\left(g_{y^{\prime}}(w, y), y^{\prime}\right) \\
& =\quad \text { if } g_{y^{\prime}}(w, y)>U^{A u t}\left(y^{\prime}\right)
\end{aligned}
$$

together with the constraint

$$
(1-\beta) h(w, y)+\beta \sum_{y^{\prime} \in Y} \pi\left(y^{\prime} \mid y\right) g_{y^{\prime}}(w, y)=w
$$

to solve for solve $N+1$ equations $^{27}$ for the $N+1$ optimal policies $g_{y^{\prime}}^{0}(w, y)$ and $h^{0}(w, y)$. Notice that $g_{y^{\prime}}^{0}(w, y)$ and $h^{0}(w, y)$ are not constrained to lie in $G$. Carrying out this procedure for all $w, y \in G \times Y$ defines $g_{y^{\prime}}^{0}(.,$.$) and h^{0}(.,$.$) that are piecewise linear functions in w$.

We then use envelope condition

$$
V_{1}^{\prime}(w, y)=\frac{(R-1)}{R(1-\beta)} C^{\prime}\left(h^{0}(w, y)\right)
$$

to update our guess of $V^{\prime}$ and repeat the procedure until convergence of $g_{y^{\prime}}^{n}(.,),. h^{n}(.,$. and $V_{n}^{\prime}(w, y)$ is achieved. This yields policy functions that are piecewise linear in $w$.

\footnotetext{
${ }^{27}$ Note that whenever the first order condition does not hold with equality we know that $g_{y^{\prime}}(w, y)=U^{A u t}\left(y^{\prime}\right)$ and we can drop the first order condition for the specific $y^{\prime}$ as the number of unknowns is reduced by 1 .
} 
To compute the stationary joint measure over $(w, y)$ we proceed as follows: for a given $(w, y)$ we find $w_{y^{\prime}}^{l}(w, y), w_{y^{\prime}}^{h}(w, y)$ and $\alpha_{y^{\prime}}(w, y)$ such that

- $w_{y^{\prime}}^{l}(w, y)=\max \left\{w \in G \mid w \leq g_{y^{\prime}}(w, y)\right\}$

- $w_{y^{\prime}}^{h}(w, y)=\min \left\{w \in G \mid w>g_{y^{\prime}}(w, y)\right\}$

- $\alpha_{y^{\prime}}(w, y)$ solves $\alpha_{y^{\prime}}(w, y) w_{y^{\prime}}^{l}(w, y)+\left(1-\alpha_{y^{\prime}}(w, y)\right) w_{y^{\prime}}^{h}(w, y)=g_{y^{\prime}}(w, y)$.

We then define the Markov transition matrix $Q:(G \times Y) \times(G \times Y) \rightarrow[0,1]$ as

$$
Q\left((w, y),\left(w^{\prime}, y^{\prime}\right)\right)=\left\{\begin{array}{lrr}
\pi\left(y^{\prime} \mid y\right) \alpha_{y^{\prime}}(w, y) \quad \text { if } & w^{\prime}=w_{y^{\prime}}^{l}(w, y) \\
\pi\left(y^{\prime} \mid y\right)\left(1-\alpha_{y^{\prime}}(w, y)\right) \text { if } & w^{\prime}=w_{y^{\prime}}^{h}(w, y) \\
0 & \text { else } &
\end{array}\right.
$$

Note that the matrix $Q$ has dimension $(K \cdot N) \times(K \cdot N)$. We then solve the matrix equation

$$
\Phi=Q^{T} \Phi
$$

for $\Phi$, where $\Phi$ has dimension $K \cdot N$ and $\Phi(w, y)$ gives the steady state probability of being in state $(w, y)$. In this way we can find, for a given $R \in\left(1, \frac{1}{\beta}\right), \Phi_{R}, h^{R}(w, y)$ and $g_{y^{\prime}}^{R}(w, y)$. We then compute the excess demand function

$$
d(R)=\sum_{(w, y) \in G \times Y}\left(C\left(h^{R}(w, y)\right)-y\right) \Phi_{R}(w, y)
$$

and use a Newton procedure to find $R$ such that $d(R)=0$. This completes the computation of $R, \Phi_{R}$ and an allocation induced by the policies $h^{R}(w, y)$ and $g_{y^{\prime}}^{R}(w, y)$ that satisfy the hypotheses of theorem 1 . 